28 May 2018

\title{
The D0 Silicon Microstrip Tracker
}

\author{
S.N. Ahmed,$^{i}$ R. Angstadt, ${ }^{v}$ M. Aoki, ${ }^{v}$ B. Åsman, ${ }^{k}$ S. Austin, ${ }^{v}$ L. Bagby, ${ }^{v}$ \\ E. Barberis, ${ }^{a d}$ P. Baringer, ${ }^{a a}$ A. Bean, ${ }^{a a}$ A. Bischoff,$s$ F. Blekman,${ }^{i}$ T.A. Bolton, ${ }^{a b}$ \\ C. Boswell, ${ }^{s}$ M. Bowden, ${ }^{v}$ F. Browning, ${ }^{v}$ D. Buchholz,${ }^{y}$ S. Burdin,${ }^{v}$ D. Butler,${ }^{v}$ \\ H. Cease, ${ }^{v}$ S. Choi, ${ }^{s}$ A.R. Clark, ${ }^{p}$ J. Clutter, ${ }^{a a}$ A. Cooper ${ }^{v}$ W.E. Cooper, ${ }^{v}$ \\ M. Corcoran, ${ }^{a n}$ S.J. de Jong ${ }^{i}$ M. Demarteau, ${ }^{v}$ R. Demina, ${ }^{a i}$ S. Desai, ${ }^{v}$ G. Derylo, ${ }^{v}$ \\ J. Ellison, ${ }^{s}$ P. Ermolov, ${ }^{j, \ddagger}$ J. Fagan, ${ }^{v}$ J. Fast, ${ }^{v}$ F. Filthaut, ${ }^{i}$ J. Foglesong, ${ }^{v}$ \\ H. Fox, ${ }^{g}$ C.F. Galea, ${ }^{i}$ J. Gardner, ${ }^{a}{ }^{a}$ R.J. Genik II, ${ }^{m}$ C.E. Gerber, ${ }^{w}$ Y. Gershtein, ${ }^{a f}$ \\ K. Gounder,${ }^{s}$ S. Grinstein,${ }^{a}$ W. Gu ${ }^{v}$ P. Gutierrez ${ }^{a k}$ H. Haggerty, ${ }^{v}$ R.E. Hall, ${ }^{q}$ \\ S. Hagopian, ${ }^{u}$ R. Hance, ${ }^{v}$ K. Harder ${ }^{l}$ P. Heger,${ }^{v}$ A.P. Heinson, ${ }^{s}$ U. Heintz, ${ }^{a c}$ \\ G. Hesketh,${ }^{a d}$ D. Hover, ${ }^{a a}$ J. Howell, ${ }^{v}$ M. Hrycyk, ${ }^{v}$ I. Iashviliag M. Johnson, ${ }^{v}$ \\ H. Jöstlein, ${ }^{v}$ A. Juste, ${ }^{v}$ W. Kahl, ${ }^{a b}$ E. Kajfasz,${ }^{f}$ D. Karmanov, ${ }^{j}$ S. Kesisoglou, ${ }^{a m}$ \\ A. Khanov ${ }^{a l}$ J. King, ${ }^{a a}$ S. Kleinfelder ${ }^{t}{ }^{\text {J. Kowalski, }}{ }^{v}$ K. Krempetz, ${ }^{v}$ M. Kubantsev, ${ }^{a b}$ \\ Y. Kulik, ${ }^{v}$ G. Landsberg, ${ }^{a m}$ A. Leflat, ${ }^{j}$ F. Lehner, ${ }^{v, a o}$ R. Lipton, ${ }^{v}$ H.S. Mao, ${ }^{d}$ \\ M. Martin,${ }^{x}$ J. Mateski, ${ }^{v}$ M. Matulik, ${ }^{v}$ M. McKenna, ${ }^{v}$ A. Melnitchouk, ${ }^{a e}$ \\ M. Merkin, ${ }^{j}$ D. Mihalcea,${ }^{x}$ O. Milgrome, ${ }^{o}$ H.E. Montgomery, ${ }^{v}$ S. Moua,${ }^{v}$ \\ N.A. Naumann,${ }^{i}$ A. Nomerotski, ${ }^{v}$ D. Olis, ${ }^{v}$ D.C. O’Neil ${ }^{c}$ G.J. Otero y Garzón, ${ }^{a}$ \\ N. Parua ${ }^{z}$ J. Pawlak, ${ }^{v}$ M. Petteni, ${ }^{n}$ B. Quinn, ${ }^{a e}$ P.A. Rapidis, ${ }^{h}$ P. Ratzmann, ${ }^{v}$ \\ F. Rizatdinova, ${ }^{a l}$ M. Roco, ${ }^{v}$ R. Rucinski, ${ }^{v}$ V. Rykalin, ${ }^{x}$ H. Schellman, ${ }^{y}$ \\ W. Schmitt, ${ }^{v}$ G. Sellberg, ${ }^{v}$ C. Serritella, ${ }^{u}$ E. Shabalina, ${ }^{w}$ R.A. Sidwell, ${ }^{a b}$ \\ V. Simak, ${ }^{e}$ E. Smith, ${ }^{a k}$ B. Squires,${ }^{v}$ N.R. Stanton,${ }^{a b}$ G. Steinbrück, ${ }^{a h}$ \\ J. Strandberg, ${ }^{k}$ S. Strandberg, ${ }^{k}$ M. Strauss, ${ }^{a k}$ S. Stredde, ${ }^{v}$ A. Toukhtarov, ${ }^{v}$ \\ S.M. Tripathi, ${ }^{r}$ T.G. Trippe, ${ }^{p}$ D. Tsybychev,${ }^{a j}$ M. Utes, ${ }^{v}$ P. van Gemmeren, ${ }^{v}$ \\ M. Vaz ${ }^{b}$ M. Weber, ${ }^{v}$ D.A. Wijngaarden ${ }^{i}$ J. Wish, ${ }^{v}$ J. Womersley ${ }^{v}{ }^{2}$. Yarema, ${ }^{v}$ \\ Z. $\mathrm{Ye}^{v}$ A. Zieminski, ${ }^{z}, \ddagger$ T. Zimmerman,,$^{v}$ E.G. Zverev ${ }^{j}$ \\ ${ }^{a}$ Universidad de Buenos Aires, Buenos Aires, Argentina
}


${ }^{b}$ LAFEX, Centro Brasileiro de Pesquisas Físicas, Rio de Janeiro, Brazil

${ }^{c}$ Simon Fraser University, Burnaby, British Columbia, Canada

${ }^{d}$ Institute of High Energy Physics, Beijing, People's Republic of China

${ }^{e}$ Czech Technical University, Prague, Czech Republic

${ }^{f}$ CPPM, Aix-Marseille Université, IN2P3-CNRS, Marseille, France

${ }^{g}$ Physikalisches Institut, Universität Freiburg, Freiburg, Germany

${ }^{h}$ National Center for Scientific Research, "Demokritos", Athens, Greece

${ }^{i}$ Radboud University Nijmegen/NIKHEF, Nijmegen, The Netherlands

${ }^{j}$ Moscow State University, Moscow, Russia

${ }^{k}$ Stockholm University, Stockholm, Sweden

${ }^{l}$ STFC Rutherford Appleton Laboratory, Chilton,United Kingdom.

${ }^{m}$ Lancaster University, Lancaster, United Kingdom

${ }^{n}$ Imperial College, London, United Kingdom

${ }^{\circ}$ Radio Astronomy Laboratory, University of California Berkeley, Berkeley, California 94720, USA

${ }^{p}$ Lawrence Berkeley National Laboratory and University of California Berkeley, Berkeley, California 94720, USA

${ }^{q}$ California State University Fresno, Fresno, California 93740, USA

${ }^{r}$ University of California Davis, Davis, California 95616, USA

${ }^{s}$ University of California Riverside, Riverside, California 92521, USA

${ }^{t}$ University of California Irvine, Irvine California, 92697, USA

${ }^{u}$ Florida State University, Tallahassee, Florida 32306, USA

${ }^{v}$ Fermi National Accelerator Laboratory, Batavia, Illinois 60510, USA

${ }^{w}$ University of Illinois at Chicago, Chicago, Illinois 60607, USA

${ }^{x}$ Northern Illinois University, DeKalb, Illinois 60115, USA

${ }^{y}$ Northwestern University, Evanston, Illinois 60208, USA 
${ }^{z}$ Indiana University, Bloomington, Indiana 47405, USA

${ }^{a a}$ University of Kansas, Lawrence, Kansas 66045, USA

${ }^{a b}$ Kansas State University, Manhattan, Kansas 66506, USA

${ }^{a c}$ Boston University, Boston, Massachusetts 02215, USA

${ }^{a d}$ Northeastern University, Boston, Massachusetts 02115, USA

${ }^{a e}$ University of Mississippi, University, Mississippi 38677, USA

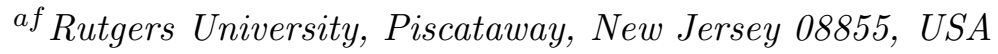

${ }^{a g}$ State University of New York, Buffalo, New York 14260, USA

${ }^{a h}$ Columbia University, New York, New York 10027, USA

${ }^{a i}$ University of Rochester, Rochester, New York 14627, USA

aj State University of New York, ?Stony Brook,? Stony Brook, New York 11794, $U S A$

${ }^{a k}$ University of Oklahoma, Norman, Oklahoma 73019, USA

${ }^{a l}$ Oklahoma State University, Stillwater, Oklahoma 74078, USA

${ }^{a m}$ Brown University, Providence, Rhode Island 02912, USA

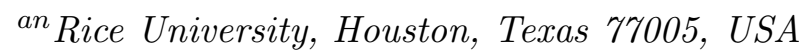

${ }^{a o}$ Visitor from University of Zurich, Zurich, Switzerland 


\begin{abstract}
This paper describes the mechanical design, the readout chain, the production, testing and the installation of the Silicon Microstrip Tracker of the D0 experiment at the Fermilab Tevatron collider. In addition, description of the performance of the detector during the experiment data collection between 2001 and 2010 is provided.
\end{abstract}




\section{Contents}

1 Introduction 1

2 General Overview

3 Mechanical Structure and Environment

3.1 Introduction

3.2 Ladders and Barrels 8

3.3 F-Disk and F-Wedge Structure

3.4 Module Support 16

3.5 H-Disk and H-Wedge Structure 16

3.6 Cooling 17

3.7 Half-Cylinder and Cover 19

3.8 Run IIa Beam Pipe 21

4 Electronics 22

4.1 Introduction

4.2 Low Noise Design 23

4.3 SVXIIe Chip 25

4.4 High Density Interconnect 30

4.5 Low Mass Cables and Adapter Card 31

4.6 Interface Board

4.7 Sequencer Controller

4.8 SVXIIe Sequencer 33

4.9 VME Readout Buffer 34

$5 \quad$ Silicon Sensors

5.1 Barrel Sensors

5.2 Disk Sensors 39 
5.3 Sensor Production and Testing 39

5.4 Micro-discharge 41

6 Production of Ladders and Wedges 44

6.1 Production of 3-, 6-, and 9-chip Ladders 44

6.2 F-Disk Wedge Production 51

6.3 H-Disk Wedge Production 53

7 Testing of Ladders and Wedges

7.1 Testing Sequence 55

7.2 Testing of the SVXIIe Chips 55

7.3 Testing of Bare HDIs

7.4 PC Based Test Stands 57

7.5 Testing of Detector Modules 59

$\begin{array}{lll}7.6 & \text { Burn-in Tests }\end{array}$

7.7 Laser Test

7.8 Diagnosis and Repair of Defective Modules 66

7.9 Electrical Grading and Characteristics of Detector Modules 68

8 Assembly of the Central Detector 70

8.1 The Barrel Assembly 70

8.2 Measurements of Ladders 72

8.3 Assembly and Survey Procedures 74

8.4 The F-disk Assembly 78

8.5 Mating of Barrels to Disks and End-disk Assemblies 81

$9 \quad$ Installation of the Detector

9.1 Transportation and Installation of the Barrel Detector 83

10 Performance and Lifetime 86 
10.1 Noise in the F-wedges 88

10.2 Performance of the Readout System 89

10.3 Radiation Damage

11 Conclusion 98

$\begin{array}{ll}\text { References } & 99\end{array}$ 


\section{Introduction}

The D0 detector [1] is one of the two detectors at the Tevatron accelerator at Fermilab. The detector performed extraordinarily well in Run I (1992-1996), as demonstrated by the discovery of the top quark [2] and many other published physics results [3].

During Run I, the Tevatron operated using six bunches each of protons and anti-protons with 3500 ns between bunch crossings. In Run II,started in 2001, it is operated with 36 bunches of protons and anti-protons with a bunch spacing of $396 \mathrm{~ns}$. The instantaneous luminosity exceeds $4 \times 10^{32} \mathrm{~cm}^{-2} \mathrm{~s}^{-1}$, and more than $12 \mathrm{fb}^{-1}$ of data are expected to be delivered in Run II. The center-of-mass energy is $1.96 \mathrm{TeV}$ in Run II compared to $1.8 \mathrm{TeV}$ in Run I.

With the expected increase in the Tevatron luminosity and the experience gained in operating the D0 detector and in analyzing data from Run I, the D0 collaboration upgraded the detector [4] to ensure the full exploitation of the physics opportunities in Tevatron Run II.

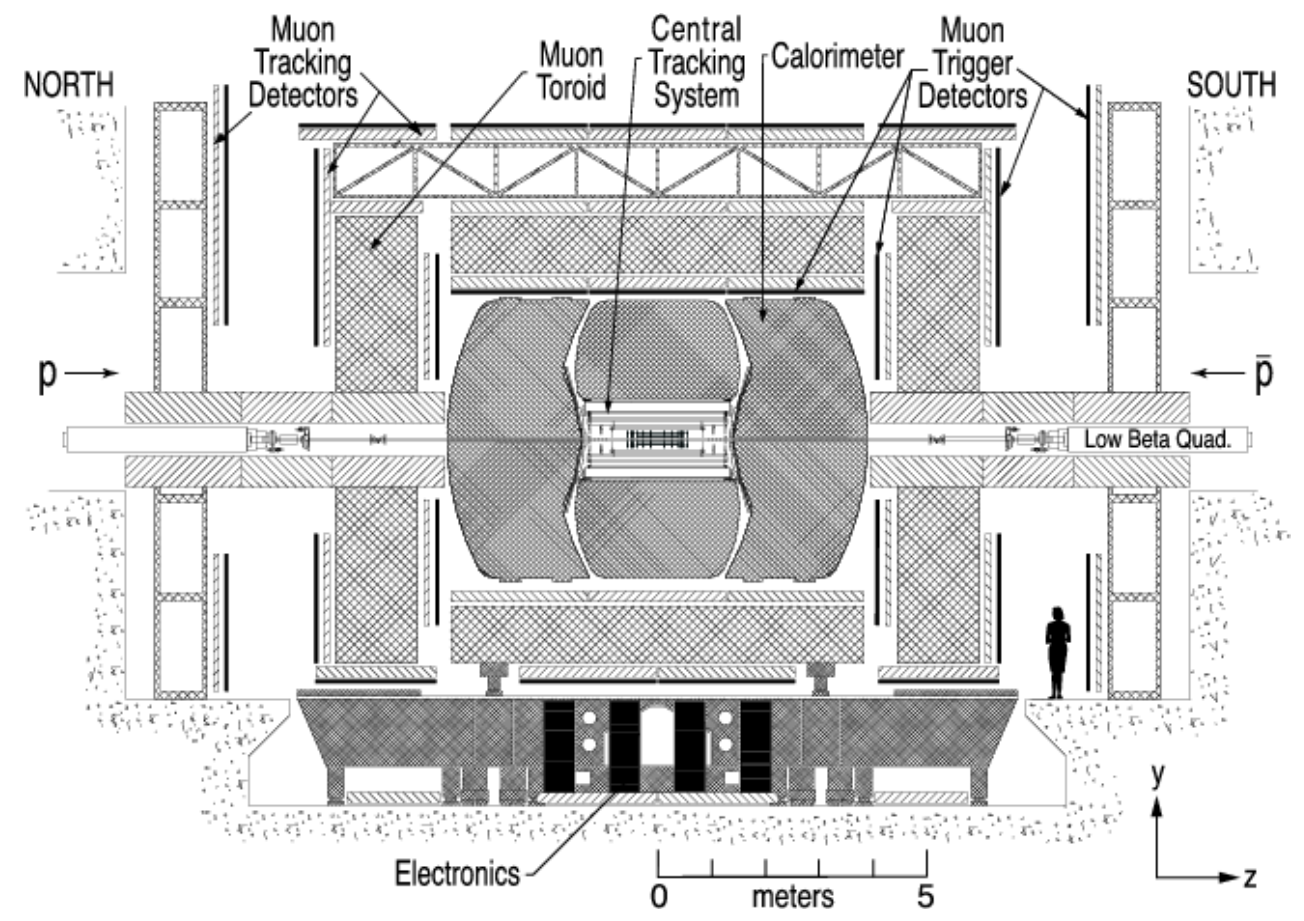

Fig. 1. Cross sectional view of the Run II D0 detector.

Figure 1 shows a cross sectional view of the Run II D0 detector. This upgrade includes faster electronics to match the reduced bunch crossing interval, upgrades of the muon system [5], and new central and forward preshower detectors to improve $e / \gamma$ identification. Most important is a completely new central tracking system, illustrated in Fig. 2, A $2 \mathrm{~T}$ axial magnetic field is provided by $\mathrm{a} \approx 2.6 \mathrm{~m}$ long superconducting solenoid magnet with $\approx 0.5 \mathrm{~m}$ 


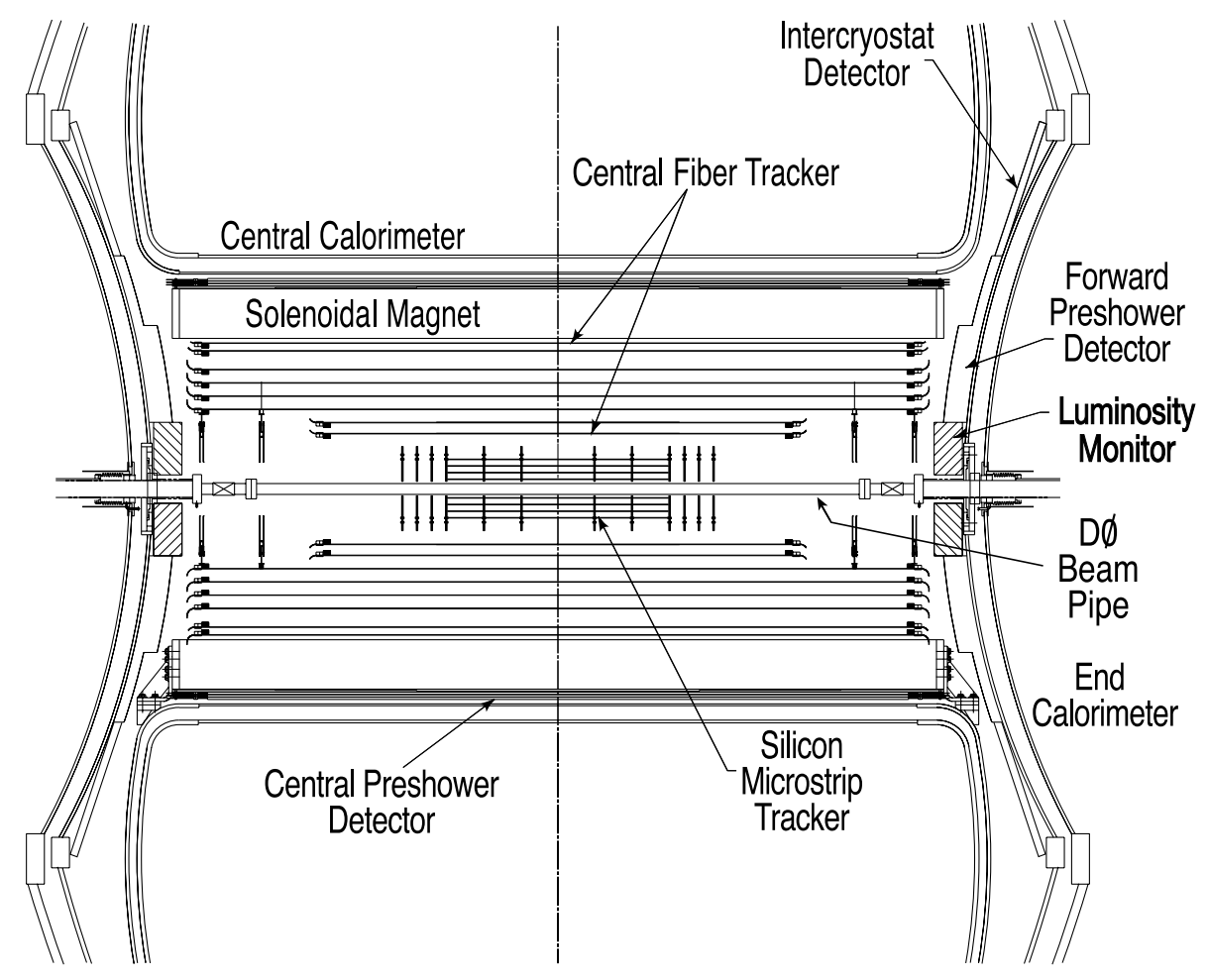

Fig. 2. Cross sectional view of the Run IIa D0 tracking system.

inner radius. The solenoid encloses a scintillating fiber tracker and a silicon microstrip tracker. The Central Fiber Tracker (CFT) consists of eight concentric barrels of scintillating fibers with axial and $\pm 3^{0}$ stereo read out. The Silicon Microstrip Tracker (SMT) is situated inside the CFT.

Since Run II has a higher instantaneous luminosity than Run I, the trigger system was also upgraded. This new system is formed by three distinct levels. The first stage, Level 1, comprises a collection of hardware trigger elements which reduces the input rate of $2 \mathrm{MHz}$ to approximately $2 \mathrm{kHz}$. The Level 2 trigger system reduces the rate further by a factor of two. In this second stage, hardware engines and embedded microprocessors associated with specific subdetectors provide information to a global processor which constructs a trigger decision based on individual objects as well as object correlations. The SMT is one of the subdetectors taking part in the Level 2 trigger. This so called Silicon Track Trigger (STT) is further described in Ref. [6]. Events that passed Level 1 and Level 2 are sent to the Level 3 trigger farm for real time reconstruction, reducing the rate to $50 \mathrm{~Hz}$.

The purpose of this paper is to provide a description of the D0 Silicon Microstrip Tracker built for Run II. In 2006 the dector was upgraded with an extra layer of silicon which was added close to the beam pipe. This is called layer 0 and is described in Ref. [7]. Furthermore the most forward and backwards disks seen in Fig. 2 where removed. The period before this upgrade is referred to as Run IIa and the period after as Run IIb. In the following section 
a general overview of the detector is given. Section 3 provides an overview of the mechanical aspects of the tracker, including a description of the cooling. This is followed by a section on the readout system. In Sec. 5, silicon wafer production and testing are described. The two following sections are about the production and quality control of the various detector components. The detector assembly is described in Sec. 8, and in Sec. 9, the installation is described. The paper concludes by summarizing some aspects of the performance of the detector.

In the description of the detector a right-handed coordinate system is used. The $z$-axis is along the proton beam direction and the $y$-axis is upward, as illustrated in Fig. 1. The angles $\phi$ and $\theta$ are the azimuthal and polar angles, respectively. The $r$ coordinate denotes the perpendicular distance from the $z$ axis. 


\section{General Overview}

The design goal of the SMT was to provide both tracking and vertexing over the full pseudorapidity $(\eta) 1$ coverage of the calorimeter and muon systems.

Several of the Tevatron machine parameters had a large effect on the SMT design. The luminosity sets a scale for the radiation damage expected over the life of the detector, which in turn dictates the operating temperature. The bunch spacing sets the design parameters for the electronics and readout, as well as the probability that multiple interactions occur in a single crossing. The length of the interaction region sets the length scale of the device. With a long interaction region of about $25 \mathrm{~cm} \mathrm{rms}$, it is difficult to deploy detectors such that the tracks are generally perpendicular to detector surfaces for all $\eta$. This feature led to the hybrid system shown in Fig. 3, with barrel detectors measuring primarily the $r$ - $\phi$ coordinate and disk detectors which measure $r$ - $z$ as well as $r-\phi$. Thus verticies for high $|\eta|$ particles are reconstructed in three dimensions by the disks, and verticies of particles at small values of $|\eta|$ are measured in the barrels. This design poses difficult mechanical challenges in the arrangement of the detector types to provide space for cooling and cables while minimizing dead areas.

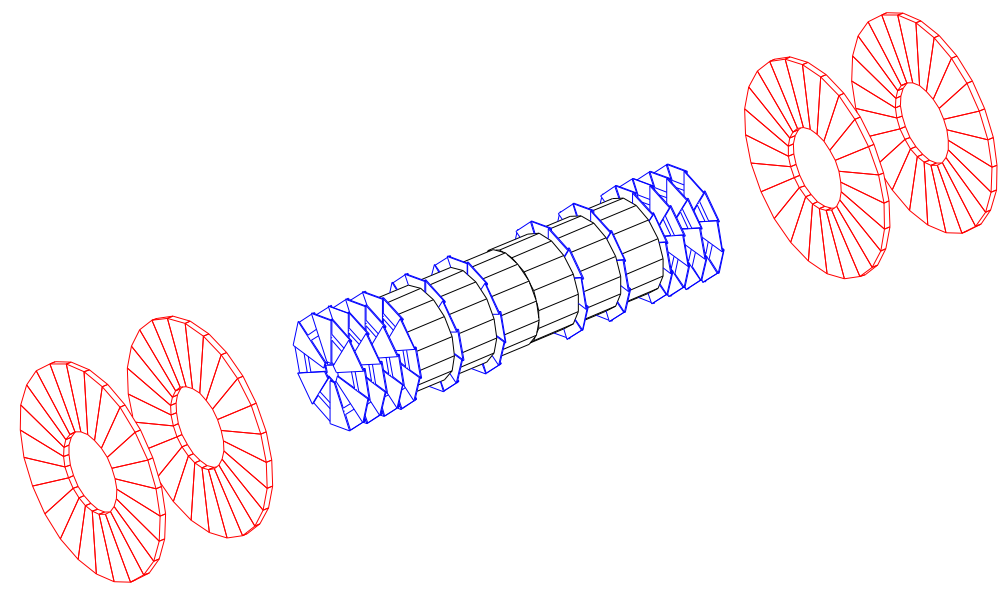

Fig. 3. Isometric view of the Run IIa D0 silicon tracker.

The design of the SMT was also influenced by the requirements of minimal mass, a precise alignment between sensors and support structures, and adequate thermal performance of the silicon modules in both the barrels and the disks. The barrel/disk design made it necessary to have the front-end electronics attached to the sensor structures. All these design constraints of the barrel silicon modules, called the ladders, led to a layout of the basic detector unit with approximately rectangular beryllium substrates with one (single-sided

1 The pseudorapidity is defined as $\eta=-\ln \left[\tan \left(\frac{1}{2} \theta\right)\right]$ where $\theta$ is the polar angle relative to the proton beam axis. 
sensors) or two (double-sided sensors) silicon surfaces glued to them. The beryllium substrates conduct heat from sensors and their on-board readout to cooling and support bulkheads. They also include features which provide precise alignment of ladder sensors to the bulkhead. A printed flex circuit, called the High Density Interconnect (HDI), carrying the readout chips and passive electronics components, was laminated onto the beryllium. A long flexible tail, called the pigtail, of the HDI allowed easy routing of the data and supply lines through the barrel structures. The readout chip, called the SVXIIe chip [8] provides 128 channels, each including a preamplifier, a 32 cell deep analog pipeline, and an 8 bit ADC.

Each of the six barrels is $12 \mathrm{~cm}$ long and has 72 ladders arranged in four layers, with each layer having two sub-layers at slightly different radii to provide azimuthal overlap, as illustrated in Fig. 4. The two outer barrels have 36 single-sided (SS) and 36 double-sided $2^{\circ}$ stereo (DS) ladders. The four inner barrels have 36 double-sided double-metal (DSDM) $90^{\circ}$ stereo and 36 doublesided $2^{\circ}$ stereo ladders. Figure 5 shows the locations of the different sensors in the six barrels. In the text the ladders are often referred to as 3-chip (SS), 6-chip (DSDM), and 9-chip (DS) ladders according to the number of SVXIIe chips mounted on them. The ladders are mounted between two precisionmachined beryllium bulkheads. The bulkhead that supports the sides of the ladders carrying the readout electronics is equipped with cooling channels.

Each barrel is capped with a disk of wedge detectors, called the F-disks. The F-disks comprise twelve wedges made of double-sided silicon wafers with trapezoidals shapes. The stereo angle of the $\mathrm{F}$-wedges is $30^{\circ}$. The $\mathrm{n}$-side is connected to 6 readout chips and the p-side to 8 chips. To provide further coverage at intermediate $|\eta|$, the central system is completed with a set of three F-disks on each end of the barrel assembly. Each disk is rotated by $7.5^{\circ}$ with respect to its neighboring disk.

In both the far-forward and far-backward region, two large diameter disks, called the H-disks, provide tracking information. The H-disks are made of 24 pairs of single-sided detectors glued back to back, giving a stereo angle of $15^{\circ}$. Each detector is read out by 6 chips. Since these disks give the last trackmeasuring points before the end calorimeters, the mass constraints on these detectors are more relaxed than for the F-disks or barrel ladders. This allowed the use of back-to-back single-sided detectors in a less compact package than the F-disks. The H-disks were designed to improve momentum resolution for tracks up to $|\eta|=3$.

For both the F-disks and the H-disks, the wedges are mounted and aligned on beryllium rings which include cooling channels. The barrels together with the F-disks are precisely mounted on two carbon fiber cylinders which meet at the nominal interaction point in the D0 detector. The four H-disks are individually 


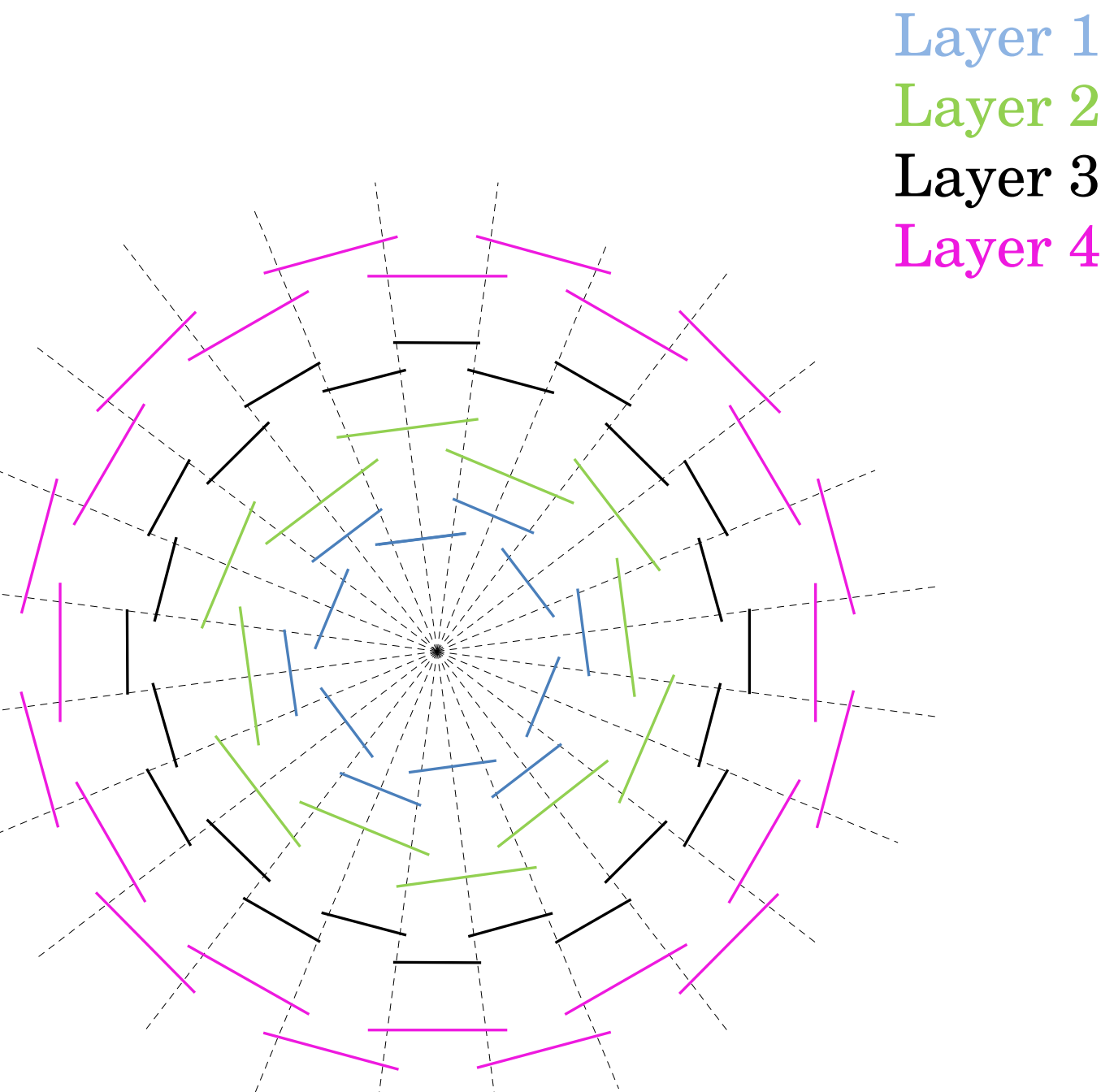

Fig. 4. Arrangement of barrel modules in $r-\phi$.

mounted on carbon fiber cylinders.

Table 1 summarizes the SMT design parameters. 


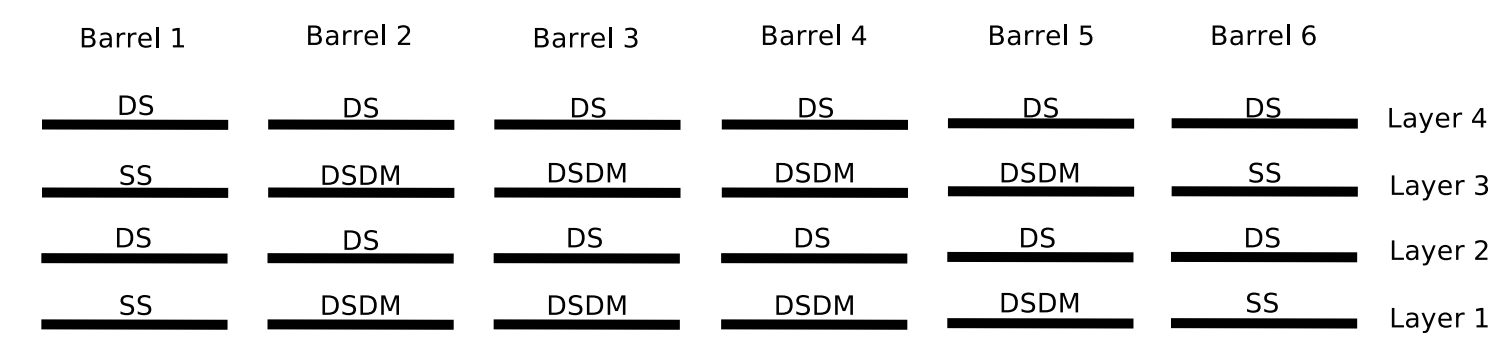

beampipe

Fig. 5. The locations of the different types of ladders.

Table 1

SMT design parameters (module refers to both ladders and wedges).

\begin{tabular}{|l|l|l|l|}
\hline & Barrels & F-disks & H-disks \\
\hline Layers/Discs & 4 & 12 & 4 \\
Channels & 387,072 & 258,048 & 147,456 \\
Modules & 432 & 144 & 96 pairs \\
Si area & $1.4 \mathrm{~m}^{2}$ & $0.4 \mathrm{~m}^{2}$ & $1.2 \mathrm{~m}^{2}$ \\
Inner radius & $2.7 \mathrm{~cm}$ & $2.6 \mathrm{~cm}$ & $9.6 \mathrm{~cm}$ \\
Outer radius & $9.4 \mathrm{~cm}$ & $10.5 \mathrm{~cm}$ & $23.6 \mathrm{~cm}$ \\
\hline
\end{tabular}

\section{Mechanical Structure and Environment}

\subsection{Introduction}

The SMT requires mechanical structures and enclosures that provide accurate positioning of the detector elements and an acceptable operating environment for the silicon. To allow optimal reconstruction of trajectories, the positions of detector elements must be known well enough that uncertainties in their positions contribute negligibly to track reconstruction inaccuracies. This placed requirements upon the position stability and upon the accuracy with which positions need to be known.

Each barrel is $120 \mathrm{~mm}$ long and $201 \mathrm{~mm}$ in diameter. The ladders are parallel to the beam pipe. The wedges are held in disks in which the planes of the wedges are normal to the beam line. The two types of disks, F-disks and H- 
disks, have active regions with outer diameter of $206.4 \mathrm{~mm}$ and of $470.1 \mathrm{~mm}$, respectively, measured along the center-lines of the sensors. Six of the F-disks are interleaved with and attached to barrels to form disk/barrel modules. The remaining disks are located at the ends of the barrel region, three F-disk and two H-disks at either end. The fiducial length of the central disk-barrel region is approximately $766 \mathrm{~mm}$, the fiducial length including the F-disks is approximately $1066 \mathrm{~mm}$ and that including the H-disks is approximately $2432 \mathrm{~mm}$. The central silicon detector, including cabling and mechanical structures, fits into a $360 \mathrm{~mm}$ diameter region. Including readout structures and protective enclosures but excluding mount structures, the total diameter of the H-disks is approximately $488 \mathrm{~mm}$.

To limit the effects of multiple scattering and the production of secondary particles and showers, it is important that the amount of material, in the mechanical structure measured in radiation lengths, is minimized. This implies the use of large radiation length materials and the minimization of the mass of the support structure in a way consistent with other design requirements.

Both the disk-barrel modules and the end F-disks are supported from two carbon-fiber-laminate cylinders of combined length approximately $1660 \mathrm{~mm}$. The two cylinders are butted against one another but electrically insulated from each another at $z=0$. In turn, they are supported from the inner surface of the innermost CFT barrel. Removable portions of the cylinders allowed modules to be installed. The cylinders also provide support for cables, cable connections, and coolant distribution manifolds. Each H-disk structure is independently supported from the inner surface of the innermost CFT barrel at large $z$ via leaf-spring, ball-and-cone kinematic mounts.

In the SMT region, the beam pipe consists of a beryllium cylinder with an outer diameter of $38.1 \mathrm{~mm}$ and an overall length of $2378 \mathrm{~mm}$.

To control noise and leakage currents in the high radiation environment, sensors are indirectly cooled by forced flow of an ethylene glycol-water mixture. The coolant temperature is typically $-8^{\circ} \mathrm{C}$.

\subsection{Ladders and Barrels}

Barrel silicon sensors are mounted on ladders which serve four purposes:

(1) They fix the relative positions of pairs of silicon sensors and provide features which allow the sensors to be accurately positioned in the barrel.

(2) They aid in flattening the sensors and in maintaining flatness.

(3) They hold the HDIs.

(4) They provide a path by which the silicon and HDI components can be 
cooled.

As mentioned in Sec. 2, three different types of ladders are used: 3-chip ladders, 6-chip ladders which have the same width as the 3-chip ladders, and 9-chip ladders which are wider. While the sensors for the 6-chip ladders are $120 \mathrm{~mm}$ long, the 3-chip and 9-chip ladders are made of two $60 \mathrm{~mm}$ long rectangular silicon sensors mounted end-to-end. A silicon barrel comprises four ladder layers at different radii; each layer consists of an inner and an outer sub-layer. A summary of ladder dimensions is given in Table 2.

Table 2

Ladder dimensions ( $\mathrm{mm})$

\begin{tabular}{|l|r|r|r|}
\hline Ladder type & 3-chip & 6-chip & 9-chip \\
\hline Single/double sided & $\mathrm{S}$ & $\mathrm{D}$ & $\mathrm{D}$ \\
\hline Stereo Angle (degrees) & none & 90 & 2 \\
\hline Silicon thickness & 0.300 & 0.300 & 0.300 \\
\hline Length of each sensor & 60.0 & 120.0 & 60.0 \\
\hline Overall length & 120.125 & 120.125 & 120.125 \\
\hline Length of active portions & 116.7 & 116.7 & 116.7 \\
\hline Silicon width & 21.2 & 21.2 & 34.0 \\
\hline Active width & 19.2 & 19.2 & 32.0 \\
\hline Maximum HDI width & 25.2 & 25.2 & 38.0 \\
\hline
\end{tabular}

The mechanical structure of a 3-chip ladder is shown in Fig. 6. Two rails, each made of a fiber composite-Rohacell foam sandwich, interconnect and support the silicon sensors. A layer of carbon-boron hybrid fibers plus a layer of carbon fibers was used for each fiber composite to closely match the thermal expansion of the silicon sensors. At the HDI end, the rails were bonded to the $0.40 \mathrm{~mm}$ thick beryllium substrate which provides reinforcement in the HDI region and aids in conducting heat from the HDI components. This beryllium piece, along with another piece near the opposite end of the ladder, also provides features used in positioning and holding the ladder.

The ladders were positioned between two bulkheads, a cooled, active, bulkhead and an uncooled, passive, bulkhead. Radial positions of the silicon are shown in Table 3 and Fig. 7 . The ladders are grouped into 24 equal $\phi$ towers centered on the layer 3 ladders as shown in Fig. 4. Layers 1 and 2, the two innermost layers, have half as many ladders as layer 3. The $\phi$ positions of ladders in layers 1 and 2 have been chosen to cover two towers. Each tower includes portions of 4 ladders. 


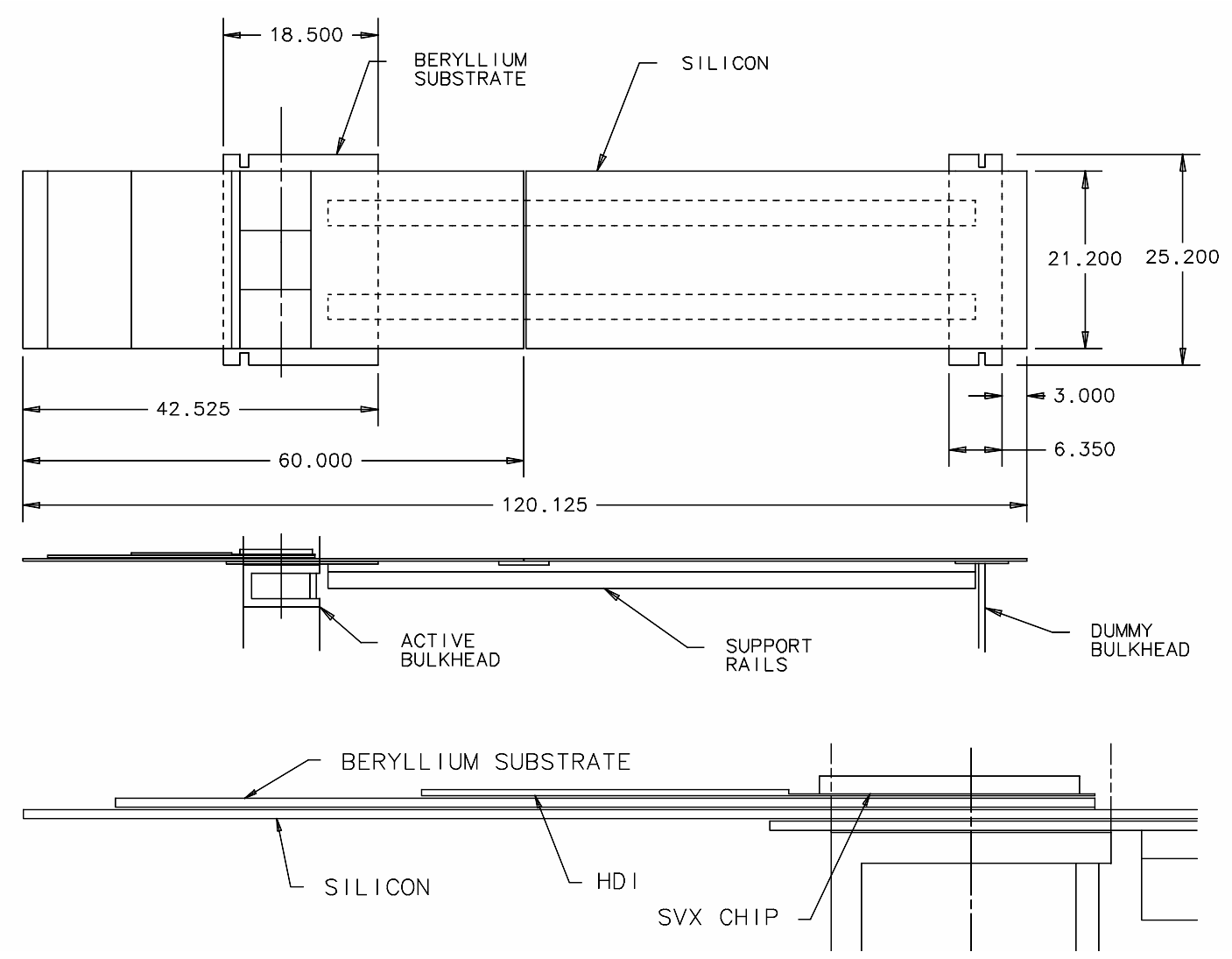

Fig. 6. Mechanical structure of the 3-Chip ladder with dimensions given in $\mathrm{mm}$. The bottom picture is a blow-up of the HDI area shown in the middle picture. The bulkheads are part of the support structure and not part of the ladder.

Several considerations drove the choice of ladder location on the bulkheads.

(1) Adequate overlap between adjacent ladders was provided to ensure complete $\phi$ coverage within each layer. This places restrictions on the maximum average radius of a layer, given the active widths and numbers of ladders.

(2) Sufficient space was provided between layers to permit ladder installation. The overall radial space for installing ladders is indicated in Tables 4 and 5. The minimum bulkhead layer-to-layer radial clearance is $10.25 \mathrm{~mm}$, allowing $1.73 \mathrm{~mm}$ clearance on both surfaces of an installed ladder, assuming a ladder already in place on the opposite sub-layer surface.

(3) The space needed for a $3.175 \mathrm{~mm} \times 7.366 \mathrm{~mm}$ cooling channel placed a lower bound on the sub-layer radial spacing.

(4) A minimum space of $5.516 \mathrm{~mm}$ was left between inner and outer sub-layer ladder mounting surfaces for cables. Components which extend from the ladder surfaces reduce the clear space for cables to about $2.7 \mathrm{~mm}$. 
Table 3

Ladder silicon radial positions along each ladders centerline.

\begin{tabular}{|c|c|c|c|}
\hline Layer & Sub-layer & $\begin{array}{c}\text { Ladders/ } \\
\text { sub-layer }\end{array}$ & $\mathbf{R}(\mathbf{m m})$ \\
\hline 1 & Inner & 6 & 27.15 \\
& Outer & 6 & 36.45 \\
\hline 2 & Inner & 6 & 45.50 \\
& Outer & 6 & 55.54 \\
\hline 3 & Inner & 12 & 67.68 \\
& Outer & 12 & 75.82 \\
\hline 4 & Inner & 12 & 91.01 \\
& Outer & 12 & 100.51 \\
\hline
\end{tabular}

Table 4

Total radial height of a ladder from the bottom up.

\begin{tabular}{|l|c|}
\hline Element & $\begin{array}{c}\text { Radial } \\
\text { height ( mm) }\end{array}$ \\
\hline Rail & 2.757 \\
Adhesive & 0.075 \\
Silicon sensor & 0.300 \\
Adhesive & 0.075 \\
Beryllium & 0.406 \\
Adhesive & 0.075 \\
HDI under chips & 0.117 \\
Adhesive & 0.075 \\
SVXIIe chip & 0.381 \\
Wire-bonds (above chip) & 0.500 \\
\hline Total & 4.761 \\
\hline
\end{tabular}

(5) Space between adjacent ladders of a sub-layer was left for a $9.4 \mathrm{~mm}$ wide layer-to-layer coolant connection. This limits the minimum radius of a layer, given ladder physical widths and the number of ladders in the 


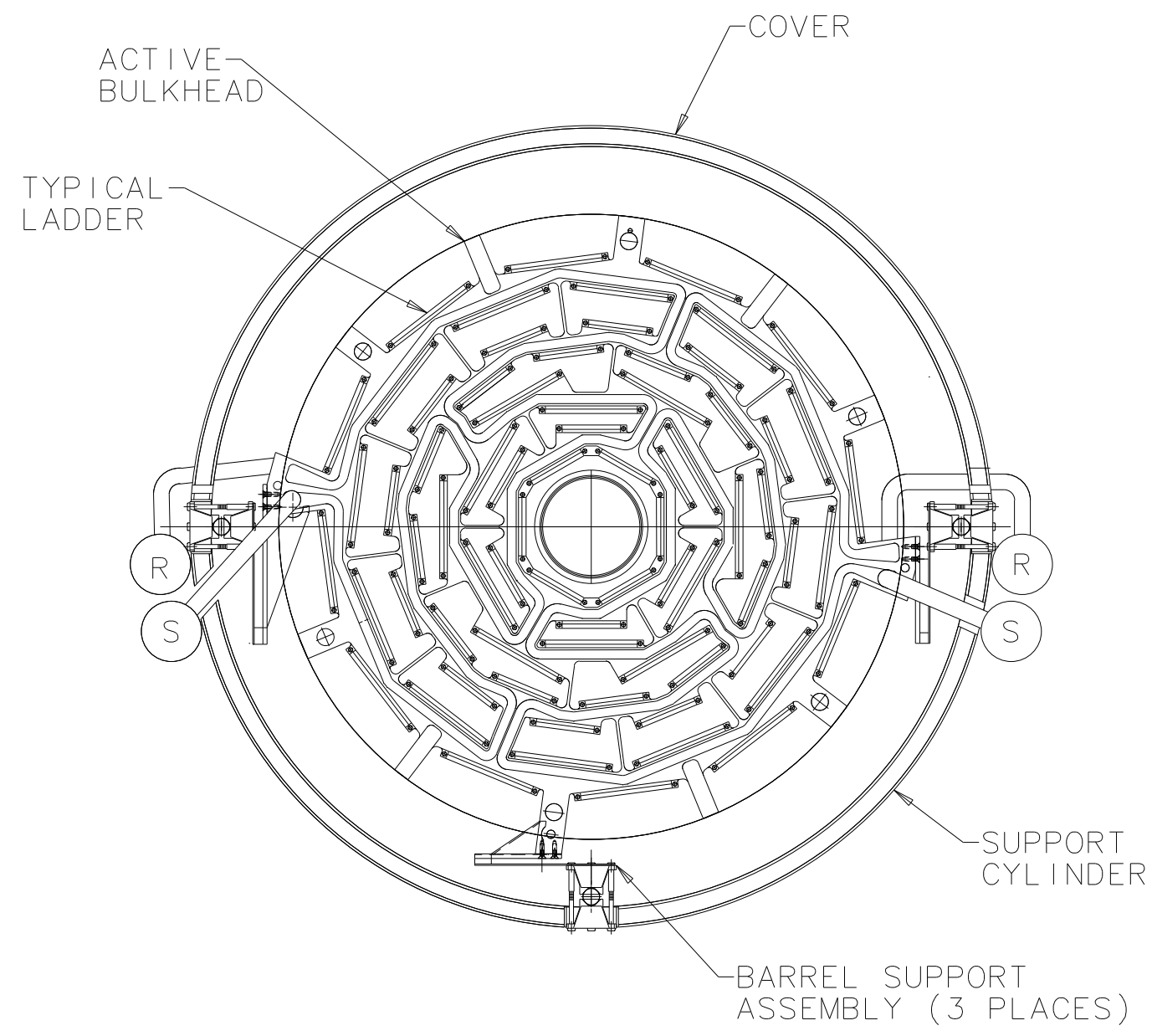

Fig. 7. Ladder positions on the active bulkhead. Four manifolds, two supply and two return, are indicated in the figure with $\mathrm{S}$ and $\mathrm{R}$.

layer.

Final machining of the active and passive bulkheads of a barrel was done with the bulkheads clamped together as a unit. This ensured that bulkhead ladder mounting surfaces and mounting features match on each pair of bulkheads and helped ensure that installed ladders would be parallel to the beam line. Mounting surfaces were machined to a flatness of approximately $25 \mu \mathrm{m}$, and the mounting surfaces on the $9.525 \mathrm{~mm}$ thick active bulkhead were made perpendicular to the plane of the bulkhead to $25 \mu \mathrm{m}$. This establishes a maximum ladder slope at the active bulkhead of 25/9525 = 2.6 mrad. Assuming this slope, perfect alignment of the active and passive bulkheads, and the appropriate elastic properties of a ladder, implies about $53 \mu \mathrm{m}$ maximum radial deflection of the ladder from a straight line. 
Table 5

Radial heights above the bulkhead mounting surface for an installed ladder.

\begin{tabular}{|l|c|}
\hline Element & $\begin{array}{c}\text { Radial } \\
\text { height ( mm) }\end{array}$ \\
\hline Adhesive & 0.051 \\
Beryllium & 0.406 \\
Adhesive & 0.075 \\
Silicon sensor & 0.300 \\
Adhesive & 0.075 \\
Beryllium & 0.406 \\
Adhesive & 0.075 \\
HDI under chips & 0.117 \\
Adhesive & 0.075 \\
SVXIIe chip & 0.381 \\
Wire-bonds above chips & 0.500 \\
\hline Total & 2.461 \\
\hline
\end{tabular}

Ladders were positioned laterally with the aid of posts on the active and passive bulkheads which extend from the bulkhead mounting surfaces and engage notches in the ladder beryllium. Only the beryllium piece closest to the bulkhead mounting surface and the edges of the posts closest to the ladder centerline were used for this purpose. Clearances between notches in the beryllium piece of the ladder and the posts were chosen so that ladders could be placed on each bulkhead with a transverse accuracy of $\pm 6 \mu \mathrm{m}$. Malleable pins through holes in the posts hold the ladder in place. In addition, the outermost layer ladders were glued in place to provide a rigid coupling between the active and passive bulkheads. The ladders play a very important structural role in the barrels. Each barrel is a complete, internally aligned unit. All barrel positioning was done with respect to the active bulkhead. The passive bulkhead is held and accurately positioned by the ladders and sets only the relative positions of ladders.

The barrel structure formed by the ladders and bulkheads must be sufficiently stiff that internal alignment criteria are satisfied under forces from the cables, the coolant connections, and thermal contraction. The required stiffness is provided without the use of additional structural members, by fastening ladders to the bulkheads with epoxy. The total barrel structure has a torsional 


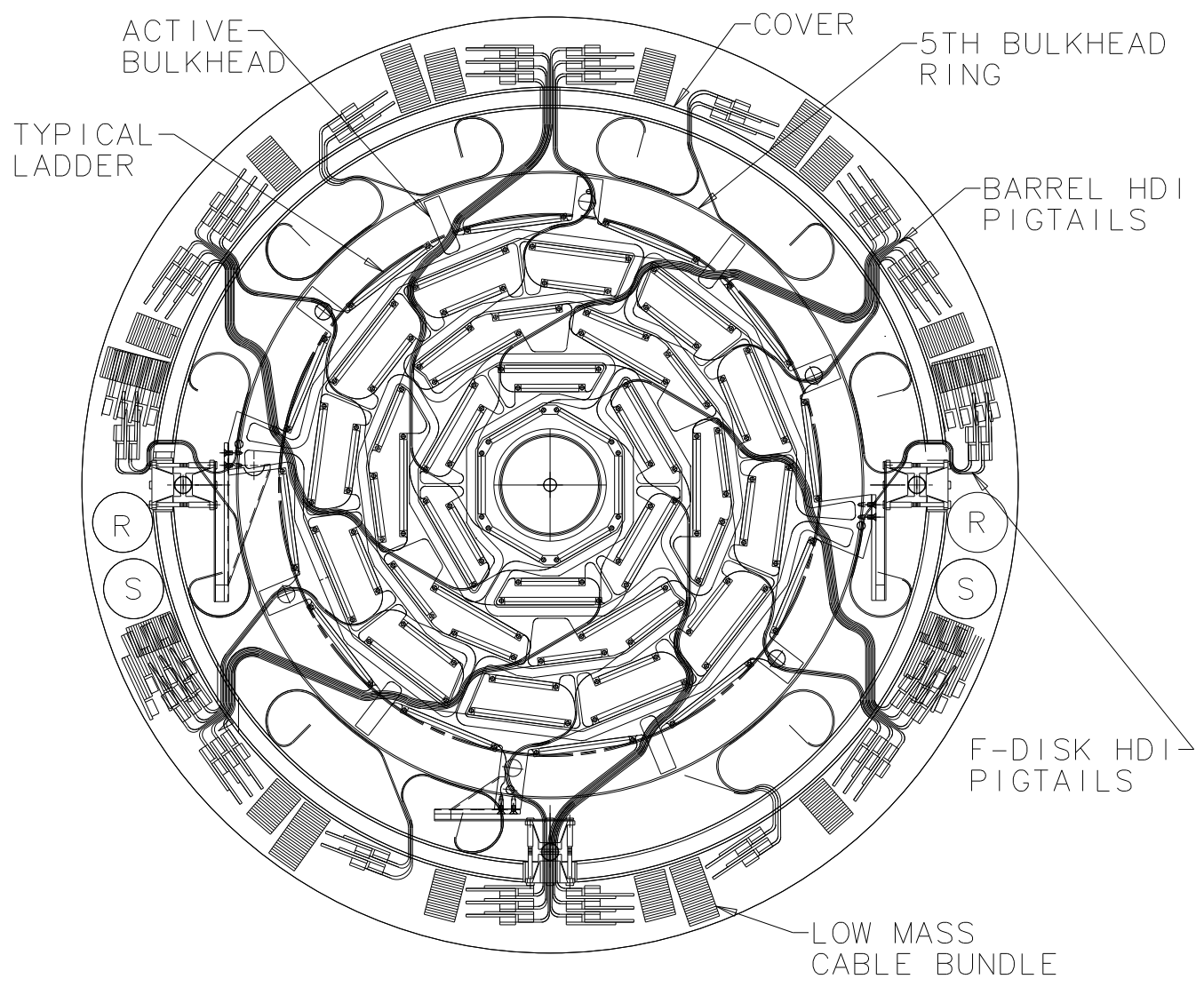

Fig. 8. Barrel cable paths.

compliance of $0.11 \mu \mathrm{m} / \mathrm{N} \cdot \mathrm{m}$ assuming that the passive bulkhead positions ladders relative to each other only in $r$ and $\phi$. Uncontrolled forces on the barrels are approximately $1 \mathrm{~N}$. This leads to a negligible shift in ladder $\phi$ positions. Shear stresses in the most highly stressed epoxy joints with a $1 \mathrm{~N}$ force applied to the passive bulkhead at a radius of $100 \mathrm{~mm}$ are $0.0050 \mathrm{MPa}$, a factor of more than 1300 below the shear strength of the electrically and thermally conductive adhesive used (e.g., TraCon 2902 silver-filled epoxy).

The pigtails that emerge at the HDI edge follow paths between and around ladders to the outer radius of the barrel, penetrate the support half-cylinder or its covers, and connect to low mass cables mounted on the outside. An extra bulkhead ring, made of carbon fiber composite, is provided at a radius of about $130 \mathrm{~mm}$ to anchor the cables. The cables are grouped at this ring into bundles which are brought through the half-cylinder and its covers at 2:00, 4:00, 6:00, 8:00, 10:00, and 12:00 o'clock as shown in Fig. 8. 


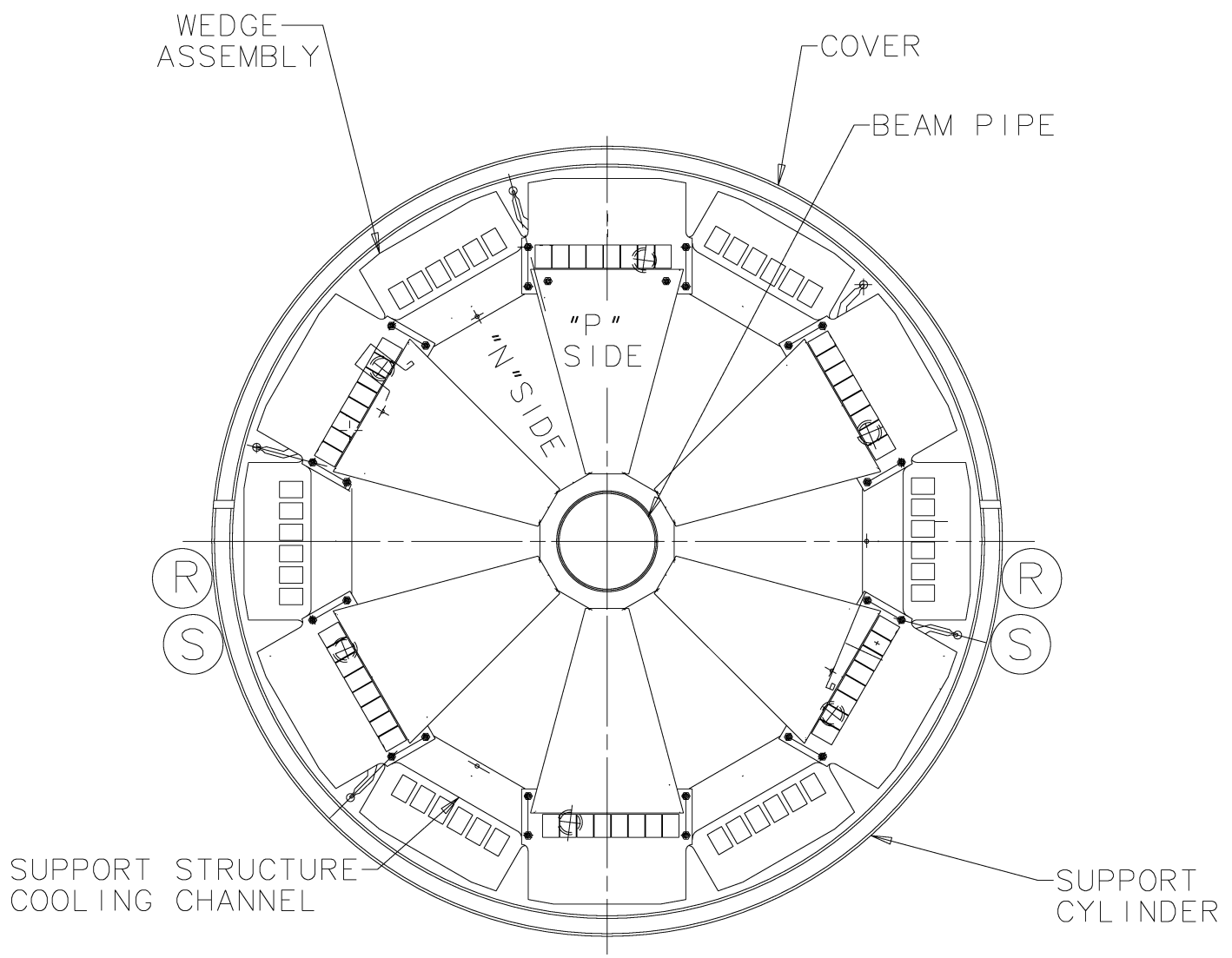

Fig. 9. F-disk detectors.

\subsection{F-Disk and F-Wedge Structure}

As mentioned in Sec. 2, each barrel is accompanied by an F-disk, and three more F-disks each are installed in the forward and backward regions. The schematic for an F-disk is shown in Fig. 9. It contains 12 double-sided wedges which are installed with alternating $\mathrm{p}$ - and n-sides. The dodecagon support structure is made of beryllium and has a cross-section of $15.7 \mathrm{~mm} \times 2.5 \mathrm{~mm}$. A $7.5 \mathrm{~mm} \times 1.0 \mathrm{~mm}$ channel in the beryllium provides a path for coolant flow to cool the wedges. Disks are oriented so that the wedges match the barrel towers.

The disk thickness contributes to the $z$-gap between adjacent barrels. To minimize this thickness, the HDI was placed at the outer periphery of the wedge. The thickness of the structure is $4.850 \mathrm{~mm}$ at the detector sensor and $6.322 \mathrm{~mm}$ at the SVXIIe chips, including $1 \mathrm{~mm}$ for two sets of wire-bonds. With additional clearance to avoid potential interference and accommodate thickness 
variations, the interleaved disks fit within a barrel-to-barrel gap of about $8 \mathrm{~mm}$.

A disk was attached to the active bulkhead of each barrel to form a disk/barrel module. This permitted the disk and barrel to be accurately matched and to be installed as a unit. Each of the interleaved disks was attached to a barrel at three points spaced evenly in $\phi$. The posts are permanently attached to the beryllium disk support structure.

\subsection{Module Support}

The disk/barrel modules and end disks were mounted from the support halfcylinders in a way which adequately resists the known gravitational forces and the somewhat variable forces from cabling and coolant connections. Provided that temperature variations are sufficiently independent of $\phi$, thermal contraction effects are predictable. The thermal expansion coefficient of beryllium, $11.6 \mathrm{ppm} /{ }^{\circ} \mathrm{C}$, leads to a radial contraction of $21 \mu \mathrm{m}$ for a $100 \mathrm{~mm}$ radius structure which is cooled from $23^{\circ} \mathrm{C}$ to $5^{\circ} \mathrm{C}$. Since the connections from disks and disk/barrel modules to the support half-cylinder are all nearly identical, the vertical center-lines of these structures move together and good relative alignment is maintained.

The support half-cylinder is described in Sec. 3.7. Carbon fiber composite mounts attached to the active bulkheads connect disk/barrel modules to the half-cylinder at 3:00, 6:00, and 9:00 o'clock as shown in Fig. 7. The 3:00 and 9:00 o'clock mounts control $y$ and $z$ positions, while the 6:00 o'clock mount controls $x$ and $z$ positions. Pairs of leaf springs allow motion in one direction while remaining stiff in the other two directions. A sapphire ball between the half-cylinder portion of the coupling and the barrel portion of the coupling permitted angular orientations to be matched during assembly; four screws around the ball allowed for adjustment and locking of the angular orientation.

\subsection{H-Disk and H-Wedge Structure}

An H-disk is shown in Fig. 10, It contains 24 wedges which alternate on each surface of a beryllium cooling pipe. Four disks were deployed for Run IIa, at $z \approx \pm 100$ and $\pm 121 \mathrm{~cm}$. In Run IIb the two outermost disks were removed. Within a disk, the alternating wedges are separated by $9.1 \mathrm{~mm}$ in $z$ and the sensors are separated by $\approx 1.2 \mathrm{~mm}$ within a wedge. The disks are supported by a ball-and-cone mount from the CFT carbon fiber support cylinder. The disk supports were installed and aligned on the CFT support cylinder using a coordinate measuring machine during the assembly of the CFT to insure precise alignment during installation of the disk assemblies in D0. 


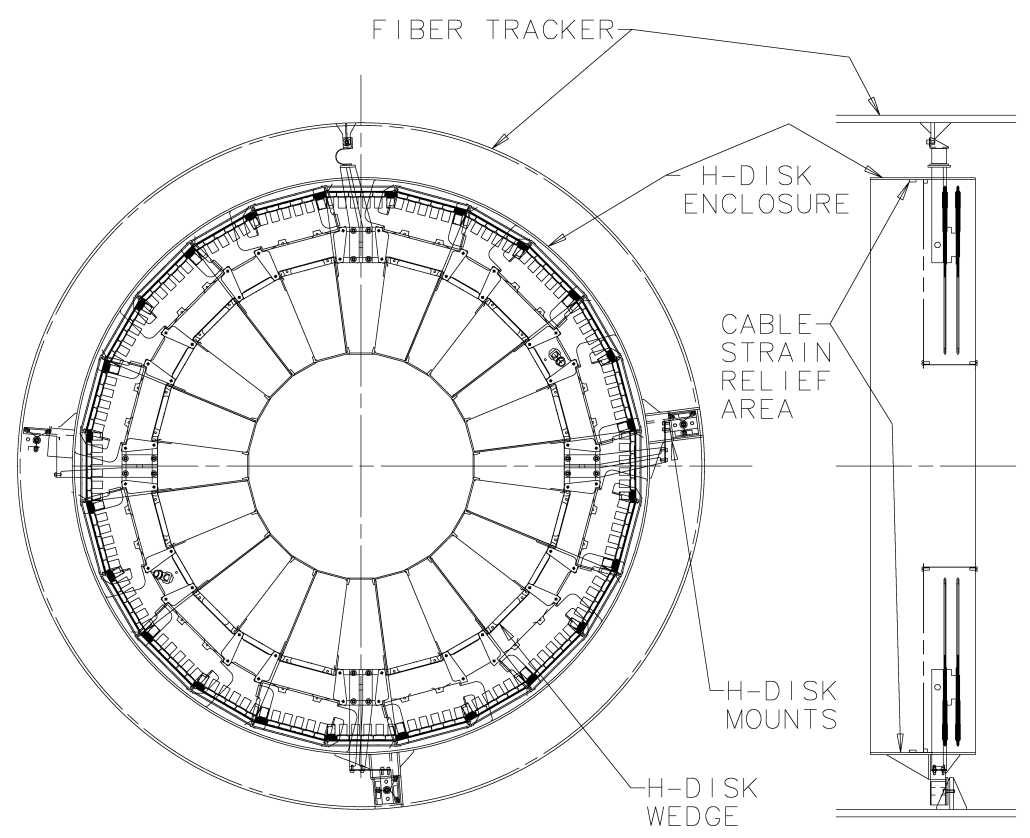

Fig. 10. H-disk assembly.

An H-wedge was fabricated from two back-to-back, single-sided, detector assemblies (Fig. 11). The inner and outer radii at the wedge centerline are $9.61 \mathrm{~cm}$ and $23.6 \mathrm{~cm}$, respectively. The detector pair is supported by a beryllium plate, which also forms the wedge support for attachment to the beryllium cooling ring. Front and back detectors on a wedge were aligned using a specially fabricated dual microscope alignment system.

\subsection{Cooling}

The heat to be removed from detector-mounted readout electronics is summarized in Table 6. The combined power dissipation for the SVXIIe chips and other HDI components is about $3 \mathrm{~mW}$ per channel. Heat loss in the cabling brings the total power dissipation to about $2400 \mathrm{~W}$.

The ethylene glycol/water cooling was chosen because of the favorable heat capacity of water and the simplicity of water systems. The cooling system consists of a reservoir, pump, flow control valve, de-ionizer, vacuum system, supply lines, return lines, and instrumentation. The pump circulates approximately $60 \mathrm{l} / \mathrm{min}$ of coolant. At this flow rate, the barrels, disks, and manifolds produce an aggregate pressure drop of $55 \mathrm{kPa}$. To prevent coolant from leaking 


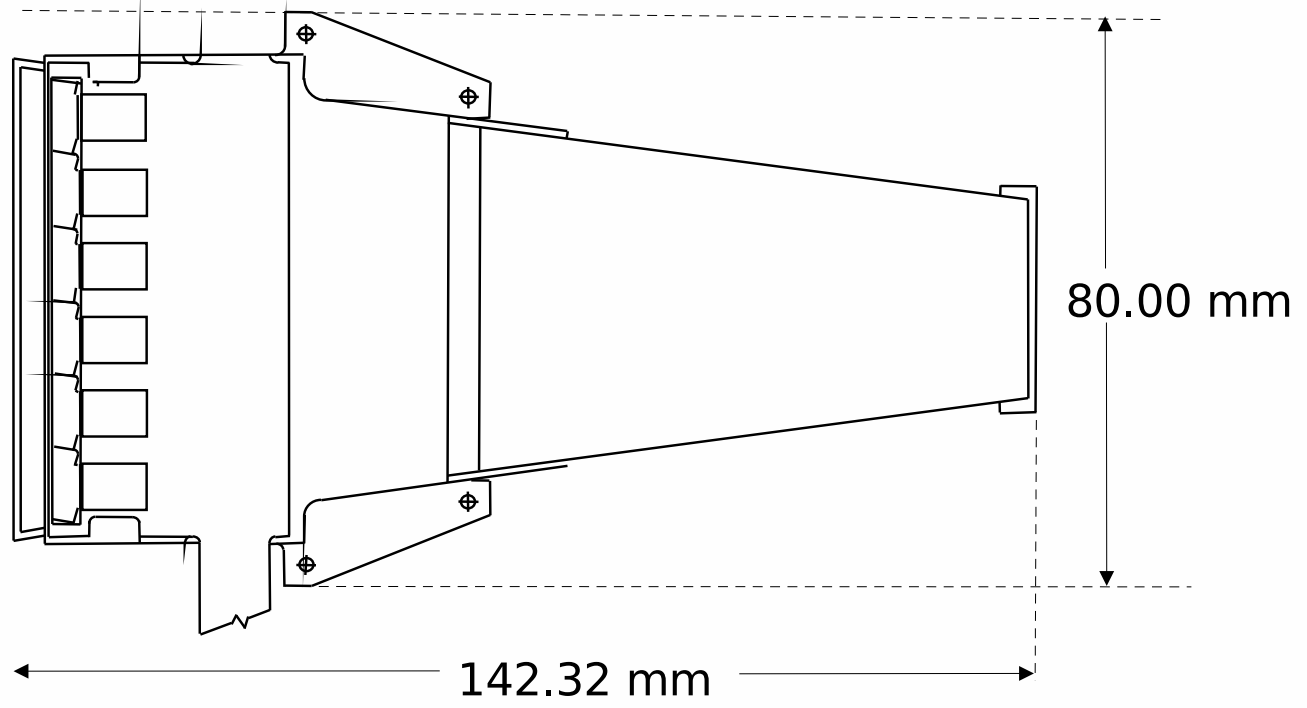

Fig. 11. H-wedge assembly.

Table 6

Detector power dissipation (watts).

\begin{tabular}{|l|c|c|c|}
\hline Elements & $\begin{array}{c}\text { Dissipation } \\
\text { (watts) }\end{array}$ & $\begin{array}{c}\text { Number of } \\
\text { Modules }\end{array}$ & $\begin{array}{c}\text { Dissipation } \\
\text { (watts) }\end{array}$ \\
\hline Barrel & chip & 1.15 per ladder & 36 per outer barrel \\
6 chip & 2.30 per ladder & 36 per inner barrel & 41 per outer barrel \\
9 chip & $3.46 . p e r$ ladder & 36 per barrel & 124 per barrel \\
\hline F-disks & 5.38 per wedges & 12 per disk & 65 per disk \\
H-disks & 2.30 per wedges & 48 per disk & 110 per disk \\
\hline Outer barrels & 165 per barrel & 2 barrels & 331 \\
Inner barrels & 207 per barrel & 4 barrels & 826 \\
F-Disks & 65 per disk & 12 disks & 778 \\
H-Disks & 110 per disk & 4 disks & 442 \\
\hline Detector total & & & 2377 \\
\hline
\end{tabular}

onto the detector components if leaks occur, the coolant pressure of the supply manifold at the detector is maintained at about $90 \mathrm{kPa}$. This pressure is achieved with an open bath on the suction side of the system from which fluid 
is drawn through the detector; the pressure drop in the piping to the detector and elevation difference between the open bath and the detector determine the pressure at the inlet to the detector. The pump is located at the floor of the collision hall to take advantage of the available heat to ensure adequate pressure on the suction side of the pump to avoid cavitation in the pump.

With $30 \%$ ethylene glycol/water mixture the system is operating at $-8^{\circ} \mathrm{C}$. The temperature within sensors varies with location from approximately $-5^{\circ} \mathrm{C}$ to $+2^{\circ} \mathrm{C}$. The cooling system was designed to allow temperature to be lowered another $7^{\circ} \mathrm{C}$ if compensation for the effects of radiation damage proves to be necessary.

Four coolant manifolds, two supply and two return, provide coolant flow to the barrels and disks. Each barrel requires two supply and two return connections, as shown in Fig. 7. The cooling channel of each layer of a barrel bulkhead is divided into equal left and right portions. The left portions of all layers are connected in series between the left supply and return manifolds. Similarly, the right portions are connected in series between the right supply and return manifolds. Layer-to-layer connections were machined as part of the bulkhead beryllium structure.

Disk coolant flow is divided between equal length upper and lower paths. The two paths share coolant connections at 3:00 and 9:00 o'clock. To equalize flow rates in the supply and return manifolds, the flow direction is reversed from one disk to the next. A set of supply and return manifolds is located on the outer surface of the half-cylinder near 3:30 o'clock; a second set is located near 8:30 o'clock. The supply manifolds enter the half-cylinder region as $15.875 \mathrm{~mm}$ inside diameter (ID) lines. Approximately $700 \mathrm{~mm}$ along the half-cylinder, after supplying the end disks and the first barrel/disk module, both lines reduce to $12.7 \mathrm{~mm}$. The return manifolds have the same configuration, starting as $12.7 \mathrm{~mm}$ ID pipe and then increasing to $15.875 \mathrm{~mm}$ ID pipe between the second and third barrel/disk modules. This configuration of reducing supply manifold size and increasing return manifold size produces evenly divided flow to the detectors. The manifolds are fabricated from thin-walled PVC pipe. Coolant connections from the disks and disk/barrel modules to the manifolds were made with flexible tubing.

\subsection{Half-Cylinder and Cover}

Carbon fiber half-cylinders (Fig. 12) provide accurate and stable support of detector elements. The half-cylinders are $1.1 \mathrm{~m}$ long, have an inside radius of $145 \mathrm{~mm}$, and outside radius of $153 \mathrm{~mm}$. The half-cylinders consist of $0.9 \mathrm{~mm}$ thick inner and outer shells joined by $0.4 \mathrm{~mm}$ thick webs. The webs are spaced 


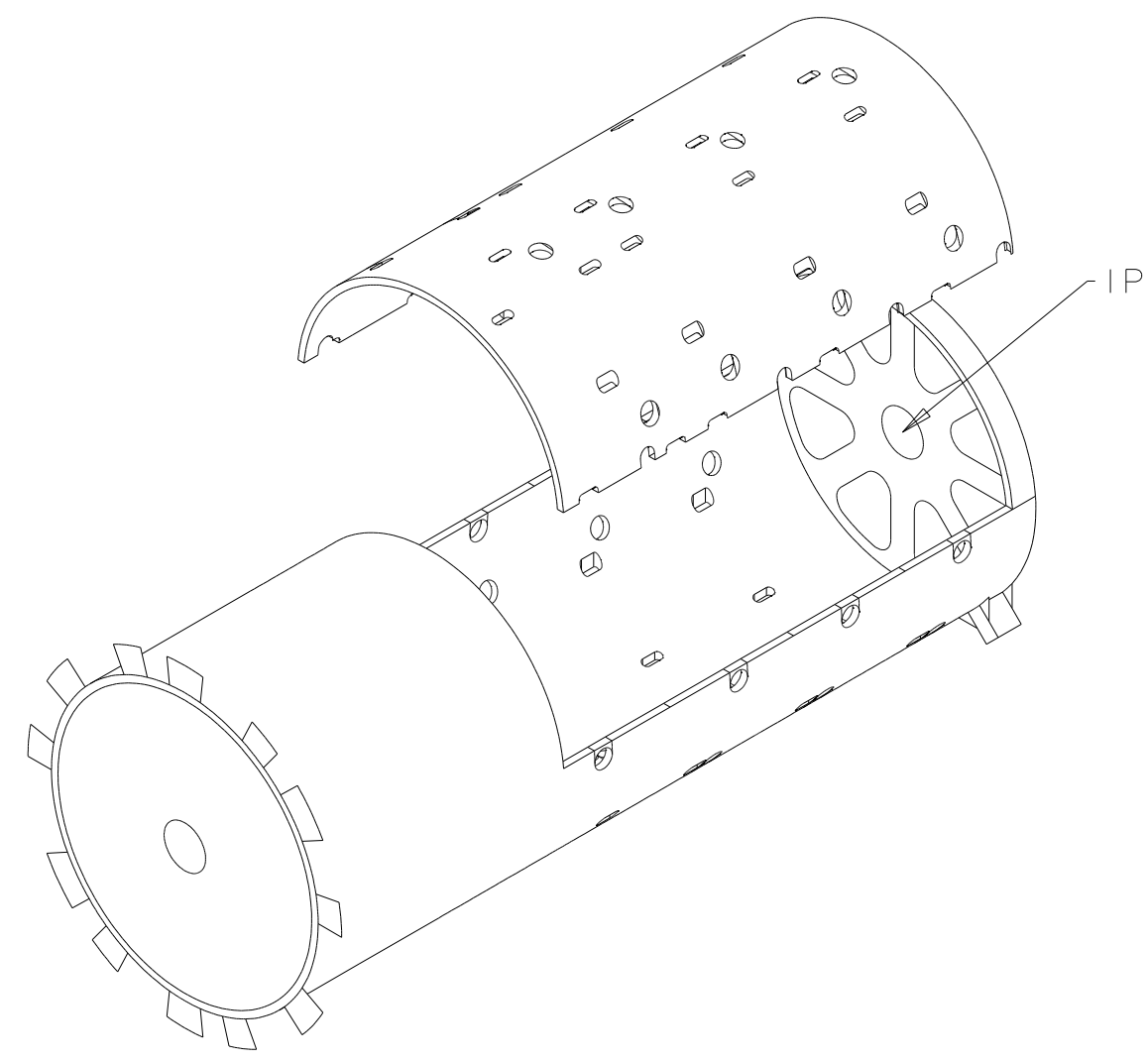

Fig. 12. Support half-cylinder and cover. IP denotes the nominal central interaction point. The holes seen on the barrel are where the cables and cooling lines pass through it. The structure on the left disk is support for the cables.

every $25.65 \mathrm{~mm}$ in $z$. The outer ends of the half-cylinders are closed by $2 \mathrm{~mm}$ thick full circular membranes which have appropriate openings for the beam pipe, cables, and coolant manifolds. The inner ends, near $z=0$, have $0.5 \mathrm{~mm}$ disks with large wedge-shaped cutouts to reduce mass in this critical region. A third circular membrane is present just beyond the outermost F-disk assembly.

The support half-cylinders were made from high modulus (900 MPa) carbon fiber prepreg plies. The high modulus carbon fiber has a low thermal expansion coefficient, high stiffness to mass ratio, reasonable cost, and acceptable fabrication properties. A low coefficient of thermal expansion ensures that temperature gradients do not cause significant displacement of detector elements in the face of the large thermal gradients from the coexistence of cooled surfaces and hot electronic chip modules. The high modulus minimizes deflections caused by gravitational, cabling, and coolant connection forces. Multiple high modulus carbon fiber plies are combined at specific angles to achieve a thermal expansion coefficient of approximately $0.9 \mathrm{ppm} /{ }^{\circ} \mathrm{C}$. The elastic mod- 
ulus of the laminate is approximately $124 \mathrm{GPa}\left(18 \times 10^{6} \mathrm{psi}\right)$. This provides a stiffness to radiation length ratio which is five times that of steel and three times that of aluminum. The high modulus carbon fiber is an excellent electrical conductor for the frequencies of concern for electronic noise (up to $1 \mathrm{MHz}$ ). The outer surface of the half-cylinder is wrapped with 2 " wide aluminum foil tape near the outer end to provide a connection from the carbon fiber to the electronics ground.

Finite element analysis, confirmed by analytical calculations, give a maximum half-cylinder deflection of $100 \mu \mathrm{m}$ and rotations about the x-axis of $70 \mu \mathrm{rad}$ at the locations of the first and last barrels. Only a fraction of this maximum deflection occurs over the length of the silicon tracker itself. Final alignment of each barrel/disk and end-disk assembly were done after the half-cylinders were fully loaded (including cables) with the cylinders supported off their final mounting points so errors due to cylinder deflection were effectively removed.

The shells and webs were made as separate components from several layers of unidirectional carbon fiber prepreg tape. Each component was formed and cured at an elevated temperature. The components were assembled to form the half-cylinder structures using room temperature curing epoxy.

The half-cylinders are supported from the CFT near $z=0$ and at $z=830 \mathrm{~mm}$. At the inner end the cylinders have two feet located at $\pm 45^{\circ}$ from the bottom with spherical ceramic ends resting in reinforced mounting points attached to the inner wall of the CFT.

Covers for the half-cylinder provide a thermal barrier, mechanical and light protection, electrostatic shielding, and a mounting surface for the low mass data cables. The covers have the same mechanical configuration as the halfcylinders of an inner and an outer skin coupled with thin radial webs. Holes are provided to allow alignment of the completed assembly.

\subsection{Run IIa Beam Pipe}

The part of the Tevatron Run IIa beam pipe that passes through the SMT has a $38.1 \mathrm{~mm}$ outside diameter and a $0.508 \mathrm{~mm}$ thick wall. Bellows and knife-edge flanges are provided at each end of the $2.578 \mathrm{~m}$ long beam pipe assembly. The Run IIb beam pipe is described in [7].

The beryllium beam pipe was fabricated by rolling a beryllium sheet into a round cylindrical shape and joining the edges with a vertical strip, providing four fillet locations for brazing. All beryllium to beryllium, and beryllium to stainless steel joints are brazed with an aluminum filler material. 


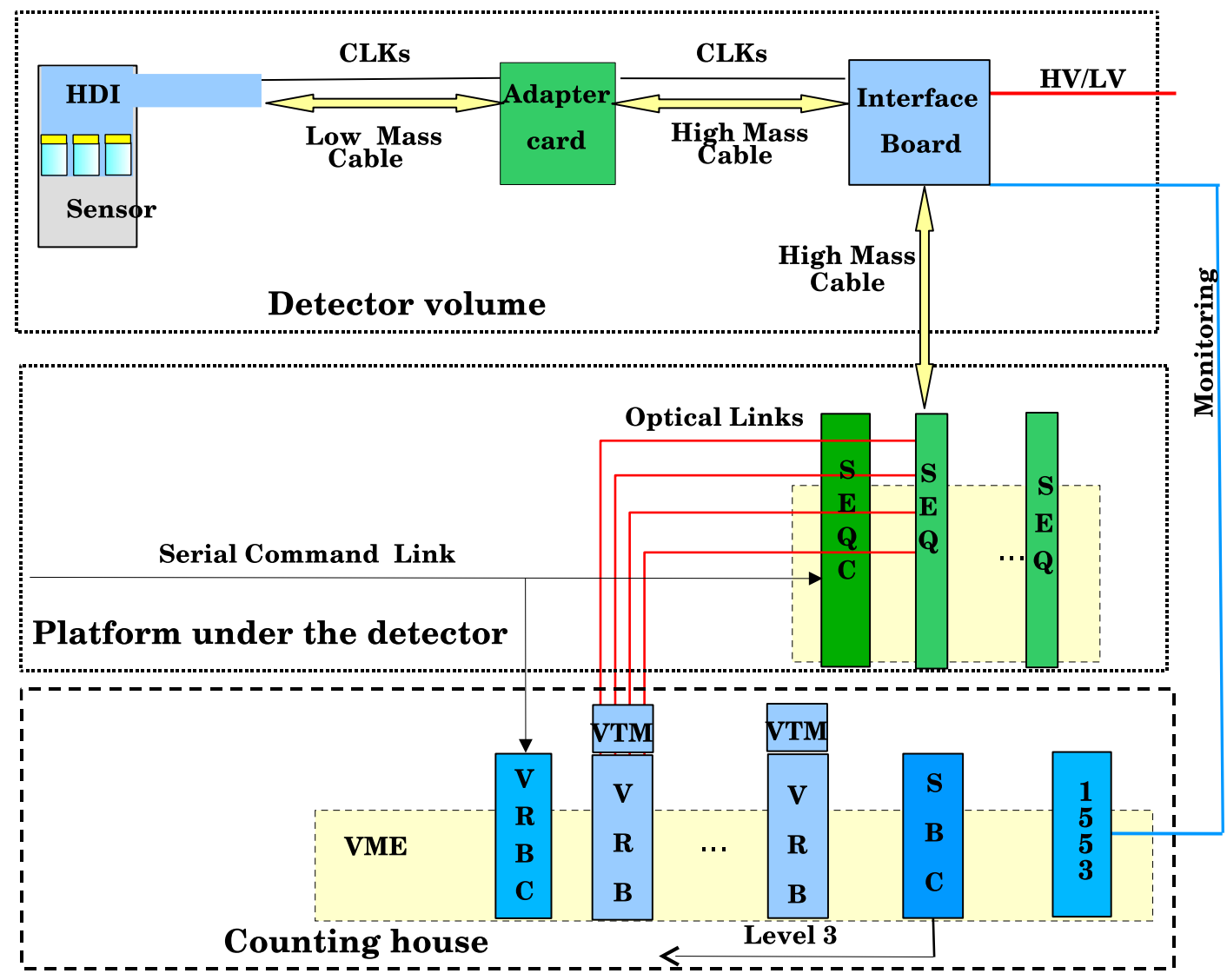

Fig. 13. D0 SMT readout system.

\section{Electronics}

\subsection{Introduction}

The data acquisition and readout system for the SMT is responsible for gathering the charge from the silicon sensors, digitizing these data, and sending it to the D0 data acquisition system. The MIL-STD-1553 connection [9] to the D0 Control System provides the ability to download and monitor the readout electronics. The readout system is depicted in the block diagram shown in Fig. 13. The locations of the components are also indicated in the figure. 
The electronics design for the SMT was dictated by the physics needs of the experiment, the engineering resources that were available, and the chip technology. The SVXIIe chip has 128 channels and a 32 deep pipeline delay to allow time for trigger formation; it can operate with $132 \mathrm{~ns}$ bunch spacing. The chips are mounted on the HDI, a polyimide hybrid. One HDI supports between three and nine chips depending on the sensor arrangement. The pigtail on the HDI allows the chip signals to be routed to the outer edge of the detector before installing a connector.

From the connector at the outer edge, a special polyimide cable, called the low mass cable, carries the signals for a distance of about $3 \mathrm{~m}$ to a small passive circuit board referred to as the adapter card. The adapter card interfaces the low mass cable to an 80-conductor 3M pleated foil cable. Two low mass cables are connected to each 80 -conductor cable. Signal dispersion in this two-cable combination limits the combined length to about $15 \mathrm{~m}$. The $15 \mathrm{~m}$ point is at the base of the liquid argon calorimeters. Since there is not enough space in this area to house the readout, a repeater board, called the interface board, was installed to retransmit the signals to the electronics area under the detector. The power supplies for the SMT are also located on the side of the central calorimeter cryostat near the interface board.

The repeated signals are sent to a sequencer module (SEQ) located under the detector on the D0 platform. The sequencer generates the sequences to download parameters into the SVXIIe chips, to put the chips into data acquisition mode, and to have the chips digitize and read out data in response to a trigger. During readout, the sequencer sends serialized data over a fiber optic link to a VME module, called a VME Read Out Buffer (VRB) [10]. The optical data are converted to 20-bit parallel data words by the VRB Transition Module (VTM) before reaching the VRB. The VRB buffers the data from each Level 1 triggers and waits for a Level 2 trigger accept. If one occurs, data are sent over the back plane to a VME readout controller and then sent over ethernet to the Level 3 processor farm.

The following sections provide more detailed descriptions of the various components and the reasons for the design choices.

\subsection{Low Noise Design}

Designing a precision, small-signal-level detector involves a complex interplay of mechanical and electrical design constraints. Many of the mechanical components are electrical conductors so they also have an affect on the electrical performance of the detector. The main goals were to minimize induced noise in the calorimeter, properly ground the beryllium mounting plates, and eliminate 
ground loops and other unwanted current paths through the detector.

The SMT is situated in the center of the calorimeter and many components are mounted on the calorimeter cryostat so preventing noise pick up required careful design. It was decided not to take additional triggers while the SMT was digitizing and reading out data, which means that when a Level 1 trigger occurs, all further Level 1 triggers are inhibited until all the data is read out from the current trigger. This results in Level 1 dead time for each Level 1 accept. The design of the SMT was simplified by this decision since the calorimeter signals are already stored in the calorimeter readout electronics when the SVXIIe digitization starts. In fact, many of the design choices such as singleended output from the SVXIIe and unshielded low mass cables would not have worked with simultaneous acquisition and digitization. Tests have shown that SMT digitization and readout generates noise signals in the calorimeter.

The only SMT signal that is active during calorimeter data taking is the clock, which is carefully shielded. The signal is sent differentially on a small, high quality coaxial cable using RF connectors. The pair of coaxial lines that are in the calorimeter bore are housed in a single braided shield, and both lines are terminated on the HDI. There is no observable noise from the clock system.

The second area of concern was the grounding of the beryllium plates. There are two important grounds for these plates. The first is the connection to the HDI. If this ground connection is not properly made, the beryllium will act as a floating capacitor, and any voltage induced on the plate will spread over the entire plate and then capacitively couple into the readout channels. This ground is achieved by bonding the ground plane on the back of the HDI directly to the beryllium with conductive epoxy. The other concern was the double sided detectors. These modules have beryllium plates on each side of the sensor, so they form a parallel plate capacitor with the sensor in the center. Any ground loop through this capacitor will induce noise into the sensor. The original solution was to use silver epoxy to form a conducting bridge between the two pieces of beryllium. There is no direct contact in this case, so the connection is dependent on the silver epoxy. Some of these bonds became resistive so all of the modules were repaired by wiring the pieces together. The wire attachments to the beryllium were made with indium solder.

The final issue was ground loops through the SMT. The SMT is read out from both ends, and each end is locally grounded. A potential difference might occur between the two ends and current will then flow in any path connecting one end of the detector to the other. If this current flows through the signal path, the detector will see this current as noise. Therefore an electrical break was made at $z=0$ for both the detector structure and the cooling path. The cooling pipes were also electrically isolated by plastic tubes at both entrances to the SMT detector. 
The common ground point for each half of the SMT was achieved by an aluminum ring which was constructed on the face of the calorimeter with all the adapter cards mounted on it. The ring was electrically isolated from the calorimeter and the adapter cards were all grounded to the ring. The ground connection to the SMT is made through the parallel combination of all the low mass cables.

\subsection{SVXIIe Chip}

The SVXIIe chip [8] is a 128 channel, full custom, mixed analog and digital integrated circuit. The main parameters are listed in Table 7.

Table 7

SVXIIe properties.

\begin{tabular}{|l|l|}
\hline SVXIIe Property & Value \\
\hline Process technology & 1.2 micron CMOS \\
\hline Chip size & 6.3 by $8.7 \mathrm{~mm}$ \\
\hline Number of channels & 128 \\
\hline Maximum interaction rate & $132 \mathrm{~ns}$ between interactions \\
\hline Detector input capacitance & 10 to $35 \mathrm{pF}$ \\
\hline Number of bits in ADC & variable up to $8 \mathrm{bits}$ \\
\hline Number of pipeline stages & 32 \\
\hline Preamp dynamic range & $240 \mathrm{pC}$ \\
\hline Wilkenson ADC clock frequency & Maximum of $53 \mathrm{MHz}$ \\
\hline Readout clock frequency & Maximum of $26.5 \mathrm{MHz}$ \\
\hline On chip zero suppression & Yes \\
\hline On chip test pulser & Yes \\
\hline Daisy chained operation & Yes \\
\hline Simultaneous trigger and readout & No \\
\hline
\end{tabular}

The chip was designed by a collaboration of engineers at Fermilab and Lawrence Berkeley Laboratory, and it was fabricated in the UTMC radiation hard $1.2 \mu \mathrm{m}$ CMOS technology. Each channel consists of an integrating preamplifier which converts charge to voltage, a 32 deep pipeline which is implemented as a string of 32 capacitors, and an 8 bit Wilkensen type ADC. A simplified diagram of 
one of the channels is shown in Fig. 14. All 128 channels are connected to a data readout section which can either read out all channels, just those above a digitally set threshold, or those above threshold plus one channel on either side of those above threshold. The latter mode can cross chip boundaries to adjacent chips mounted on the same HDI. The integrator integrates charge continuously until it is reset, but the charge into each pipeline capacitor is only the increment in charge between beam crossings. This means that there is a correlated double sampler between the integrator and the pipeline, so that only the change in charge between the previous beam crossing and the current beam crossing is put into a pipeline capacitor. The pipeline is controlled by two counters which are configured as ring counters which means that the pipeline is a ring of capacitors. There is a write counter which points to the next available capacitor in the ring. When a new beam crossing occurs, the value of the input signal is written into the capacitor pointed to by the write counter. The read counter is identical to the write counter, but it is delayed by a down-loadable pipeline delay. The beam crossing clock that samples the charge also steps the write pointer and the read pointer ring counters. If there is a trigger, the counters are stopped and the capacitor pointed to by the read counter is digitized and read out.

The SVXIIe was designed to minimize both power consumption and detector mass. It consumes approximately $3 \mathrm{~mW}$ per channel and has only 19 connections to the outside world. This small number of connections was achieved by making the function of many of the lines mode dependent. For example, the 8 bit readout bus is used for control functions in all modes but readout.

A portion of the SVXIIe schematic containing the dynamic memory cell is shown in Fig. 15.

The chip has four modes of operation with only one mode active at a time. This means that it is not possible for the chip to acquire data at the same time it is digitizing or reading out other data.

The first mode is the download mode where settable parameters are loaded into the chip. There are 190 bits in the download register for each chip. The download is implemented as a long serial shift register using only two chip pads called top neighbor and bottom neighbor and the clock. Data are shifted into the top neighbor pad and out of the bottom neighbor pad. The output from the last chip's bottom neighbor is read back into the sequencer. Thus, a three chip HDI requires sending 570 bits over the MIL-STD-1553 link [9]. The chip does not have a read command to read the data back. The data shift through the chip string two or more times and the sequencer compares the read-back data to the input.

The second mode is acquire. In this mode, every time a clock pulse arrives, the 


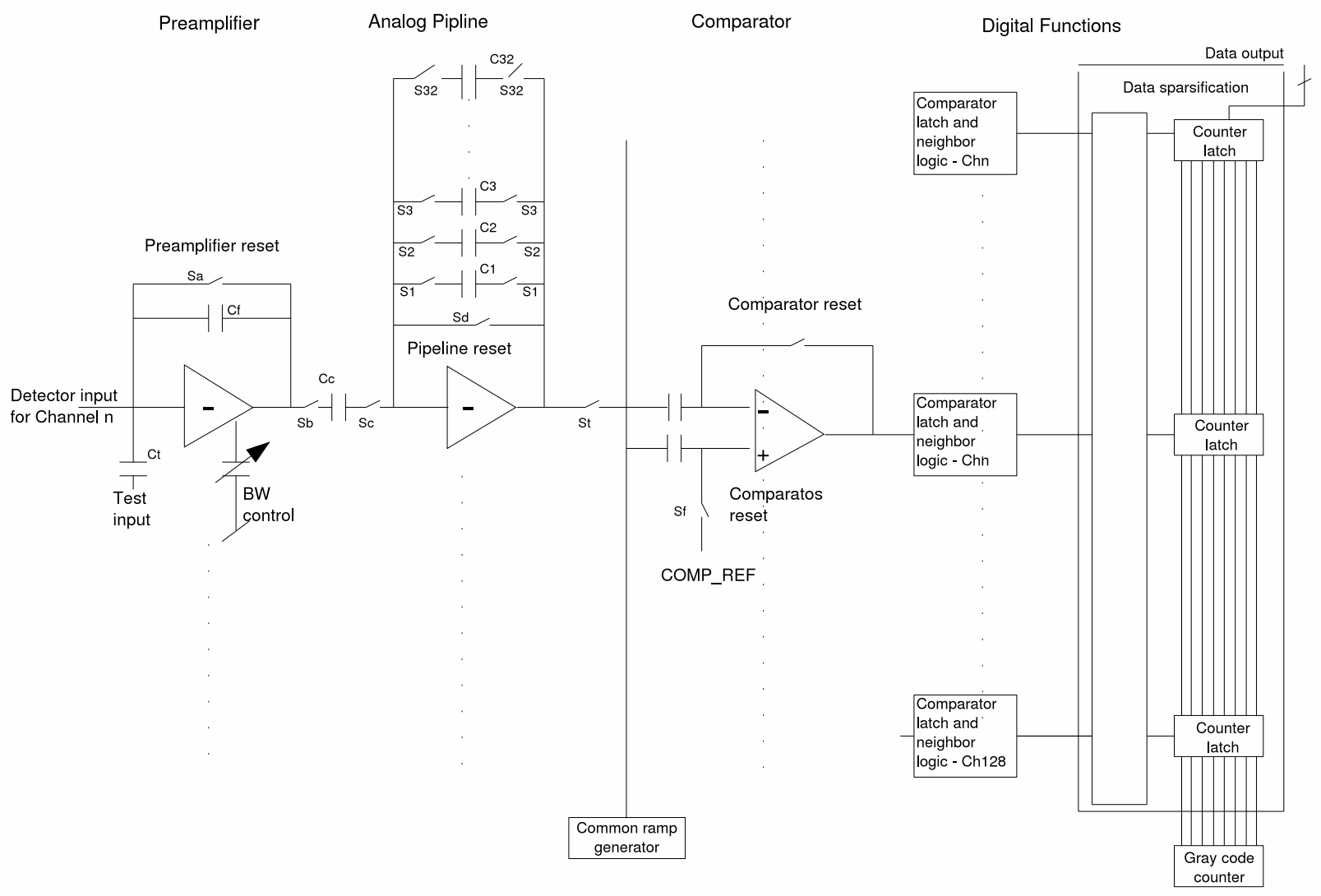

Fig. 14. A block diagram of a single channel of the SVXIIe chip.

chip samples the change in charge on the input capacitor and stores the data into a pipeline capacitor. The ring counter pointers are incremented and the process is repeated. There is no specific trigger line. When a trigger occurs, the sequencer stops the beam crossing clock and changes the mode to digitize. The correct capacitor to digitize is pointed to by the read counter.

Digitization is accomplished by transferring the charge from the selected capacitor onto a capacitor connected to a comparator. The pipeline capacitor is then reset and the offset voltage is subtracted from the charge on the comparator capacitor. This minimizes any offsets that may be present in the pipeline. Actual digitization is accomplished by putting a linear voltage ramp on the other input to the comparator and starting a counter at the same time. The $53 \mathrm{MHz}$ digitization clock increments this counter on both edges of the clock pulse giving an effective clock rate of $106 \mathrm{MHz}$. When the ramp voltage reaches the input value, the counter is stopped.

The final mode is readout. The counter values are shifted into a pipeline and the pipeline is compressed if zero suppression is enabled. Since the chip is 


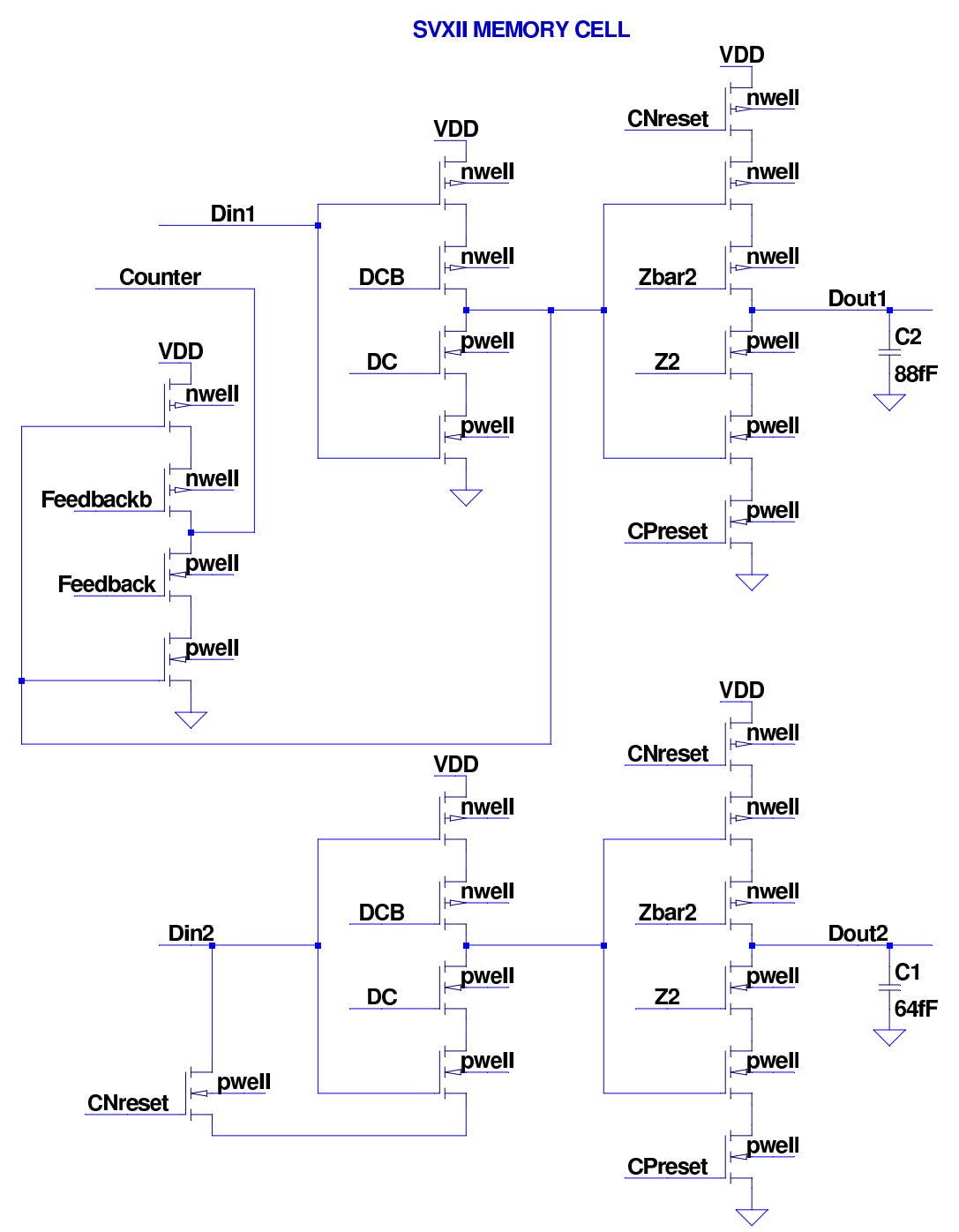

Fig. 15. A portion of the SVXIIe schematic showing the dynamic memory cell.

designed to be used in a multi-chip configuration, the readout bus is configured as a tri-state bus during readout. Control of the bus is passed between chips through the top neighbor and the bottom neighbor connections. A signal on the top neighbor during readout gives the chip control of the output bus. When it is finished it signals the bottom neighbor to give control to the next chip or, if it is the last chip, to tell the sequencer that readout is done.

Once the pipeline has been compressed and the chip has control of the output 
bus, the data is read out, one byte at a time. A 7 bit channel ID and an 8 bit data word are read out on each cycle. The clock is run at one-half of the normal $53 \mathrm{MHz}$, and data is read out on both edges of the clock. This reduces the bandwidth requirement on the readout cable but it places severe restrictions on the clock duty cycle. Small changes in the clock duty cycle can cause one edge of the data strobe to be missed which then interchanges the channel ID and data for the rest of the readout. In sparsification mode, when only channels over a threshold are read out, this error is not easily detected.

The SVXIIe has a sophisticated method for testing itself and the entire downstream readout electronics. The test circuit, called cal_inject, consists of a capacitor connected by a switch to each of the front end channels at the input to the preamplifier. The switch is activated through a transition on bus line 7 in acquire mode. The 128 bit cal_inject mask (1 bit per channel) is a down-loadable parameter so all possible patterns can be generated. The pulse amplitude can either be set by an external source or from an internal one that is controlled by a 3 bit DAC. The DAC setting is also a down-loadable parameter.

All of the power connections are at the back of the chip, including the power for the front end amplifiers. The chip's metal layers have too-high resistance for the front end amplifiers to get enough power. A row of double wire bonds was made along exposed sections of the upper metal layer connections for front end amplifier power to reduce this resistence by creating parallel paths for current flow. The spacing of the exposed sections kept the wire bond distances reliably short.

Even though the average chip power consumption is low, the current varies by nearly a factor of two between an idle state and readout, so if there is no local monitoring of the voltage by the power supply, often referred to as remote sensing, there could be a substantial change in chip voltage. The layout of the system makes remote sensing quite difficult. One supply feeds a large number of HDIs. Two HDIs are fed through the 80-conductor cables. These cables have many different lengths since they had to be as short as possible in order to fit in the space between the central and end cryostats. Finally, the number of chips varies between HDIs. The best option was to locate the power supplies as close to the detector as possible. The place with adequate space was at the base of the central calorimeter near the interface boards. The local magnetic field is about $200 \mathrm{G}$ at this location, so the power supplies had to have magnetic shielding. In addition, safe practice required fusing each power line for each of the three voltages for groups of four strings of SVXIIe chips. This required a fuse panel with about 40 fuses at four locations at the base of the calorimeter. 


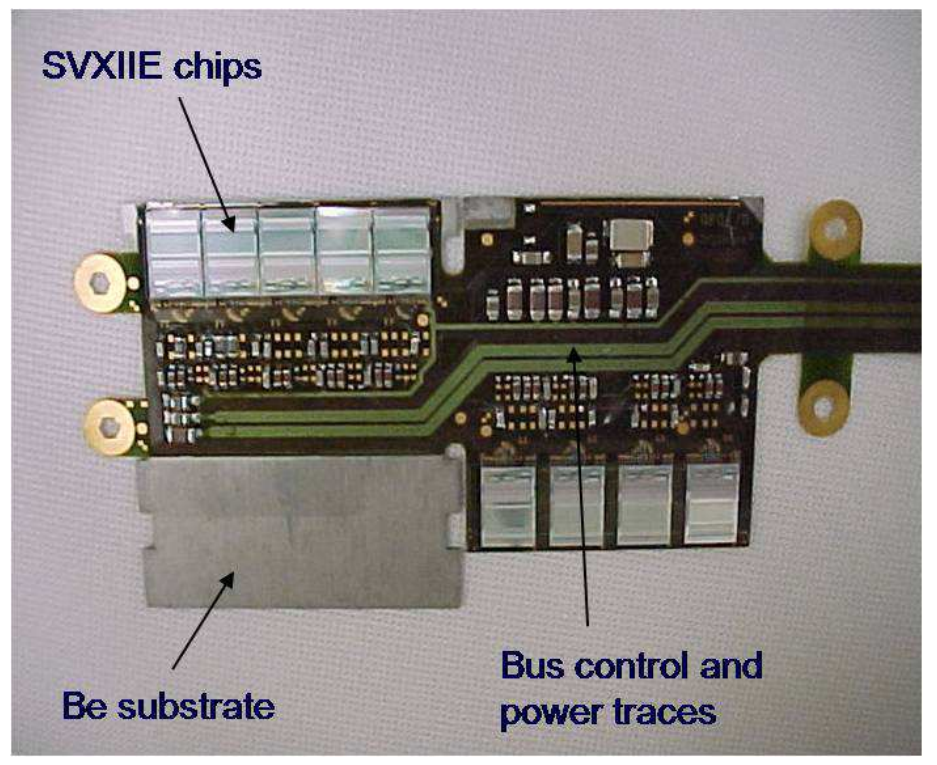

Fig. 16. An HDI for a 9-chip detector (prior to folding and lamination).

\subsection{High Density Interconnect}

The HDI is a two-layer flexible printed circuit with $50 \mu \mathrm{m}$ polyimide with $125 \mu \mathrm{m}$ line spacing and $50 \mu \mathrm{m}$ vias. The circuits are laminated to beryllium heat spreaders. One HDI and its beryllium substrate are epoxied directly to one silicon sensor. Precision notches machined into the heat spreaders provided the alignment of the silicon ladders on the bulkhead. The HDI consists of SVXIIe chips, resistors, capacitors, and an interface circuit, which are mounted in the area which are in direct contact with the silicon sensor. The SVXIIe chips are wire bonded directly to the sensors. The second part of the HDI, the pigtail, is the extension of the signal and power traces to the outer radius of the silicon detector region.

A picture of an HDI for a 9-chip detector is shown in Fig. 16.

Multiple layer polyimide circuits with very fine spacings are difficult to make because the polyimide stretches easily. A ceramic HDI would require several layers to provide the required number of signal, power, and ground planes. The small number of connections on the SVXIIe and clever use of wire bonds made it possible to make a polyimide HDI with only two layers. The output bus and control lines were laid out in a row of parallel lines in front of the string of chips. Connections from each chip to the different lines were made 
by running the wire bond over the intervening traces. Bypass capacitors were mounted outside the bus lines and longer wire bonds were used to connect to the capacitors. Thus, the equivalent of a multilayer circuit was made with wire bonds. The longest wire bonds approach the maximum allowable length so if there were many more lines to connect, this method would not have worked.

Six different types of HDIs were needed to accommodate the various detector and readout geometries. In the case of double-sided ladder detectors, a single circuit contained the readout for both the $\mathrm{p}$ - and n-sides with the polyimide folded over to sandwich the sensor. The double-sided F-wedges have one separate HDI on each side. A single HDI would have implied daisy-chaining 14 readout chips, which was considered too risky in case of a chip failure and too expensive in terms of readout time. The barrel detectors employ 9-chip HDIs for the double-sided ladders, 6-chip HDIs for the 90-degree stereo ladders, and 3-chip HDIs for the single sided ladders. The F-wedges are read out by an 8chip HDI for the p-side, and a 6-chip HDI for the n-side. Each single H-wedge is read out with one 6-chip HDI.

All HDIs have the same functionality. They provide one 8-bit data bus connecting all of the SVXIIe chips to the data path interface circuit, distribute the differential input clock and single-ended mode control signals to all the SVXIIe chips, distribute the various analog voltages required by the SVXIIe chips, and silicon bias, and provide monitoring of the temperature of the beryllium heat spreader.

\subsection{Low Mass Cables and Adapter Card}

The HDI readout cables are routed between the ladders in the barrel to a larger radius outside of the barrel vertex volume. There the pigtails are trimmed to length and attached to the low-mass cables. The low-mass cables are flexcircuit striplines, to minimize the amount of material in the sensitive volume. They were fabricated by Honeywell [11] and carry the digital signals and power between the hybrids and the adapter cards. To minimize any deterioration of the clock signals, the clock signals are brought to the hybrids through minicoaxial cables. Signal traces and broad power and ground traces are located on both sides of a polyimide dielectric about $100 \mu \mathrm{m}$ thick. The low-mass cables are routed along the half-cylinder and coupled to the 80-conductor pleated foil cables at a ring of adapter cards located between the calorimeter cryostats. Each adapter card takes the signals from two HDIs and, without further signal processing, launches them on the high quality 80-conductor pleated foil cable to the interface board. The clock signals are carried by separate coaxial cables attached to the 80-conductor cable. 


\subsection{Interface Board}

The interface board serves as a repeater for the signals between the SVXIIe and the sequencer. Each board handles up to eight detector modules. It also provides the distribution of the bias voltage to the sensors, allows remote control (on/off) of the power for the SVXIIe chips, and provides monitoring of the chip voltages, and currents.

The interface boards are housed in eight $9 \mathrm{U} \times 280 \mathrm{~mm}$ crates with custom backplanes, using 16-18 slots per crate. Two crates are placed at each of four locations at the base of the central calorimeter.

Temperatures of the beryllium heat spreaders are monitored on the interface board. A jumper on the interface board provides a selectable temperature trip at $40^{\circ} \mathrm{C}$ or $65^{\circ} \mathrm{C}$. The circuit is designed not to trip due to an open or short circuit. Calibration is adjusted by a trim resistor and is accurate to about $1^{\circ} \mathrm{C}$.

The current limiters circuits for the HDI power connections were designed to trip at $0.7 \mathrm{~A}$ in order to protect the wire bonds. Response time is a few ms. The time constant for integrated signals (output of the current monitor) is of order $100 \mathrm{~ms}$ to avoid trips on glitches. Each HDI power line is monitored, in total 24 current limiter circuits per board.

The high voltage (HV) control for the detector bias is provided by the HDI enable circuit and photovoltaic relays (no moving parts) on the interface board. There are two HV lines provided for each HDI to allow operation of doublesided sensors in split mode. HV is supplied via a 34-conductor twist-and-flat strip cable to a connector on the backplane. For single-sided detectors, the primary bias voltage is grounded and the secondary bias has a positive voltage. For double-sided detectors the primary bias is at $+\mathrm{HV} / 2$ or less and the secondary bias at $-\mathrm{HV} / 2$ or greater. This allows for asymmetric breakdown characteristics. The ground reference is the HDI ground. Bias current from groups of four HDIs can be measured via the MIL-STD-1553 interface at any time.

\subsection{Sequencer Controller}

The sequencer controller (SEQC) is a $9 \mathrm{U} \times 340 \mathrm{~mm}$ Eurostyle module residing in each sequencer crate, used to give coarse timing commands to the SVXIIe sequencers which control acquisition and readout of the SVXIIe chips. It is designed to be plugged into slot 1 of the custom sequencer backplanes.

The controller receives beam crossing timing and triggering information via 
a single coaxial cable called the Serial Command Link which originates at the Trigger Framework 2 . Information from this link is interpreted and transformed into general SVXIIe data acquisition commands that go to each sequencer via the backplane. These commands are encoded onto a serial link called the NRZ (non-return-to-zero) link which is a 7-bit repetitive code sent on dedicated backplane lines to each sequencer slot. These lines are used in conjunction with a dedicated differential $53 \mathrm{MHz}$ clock sent to each sequencer slot. The seven bits are the framing bit, the crossing bit, four bits for an encoded data command, and the parity bit. The seven bit structure was chosen to remain synchronous with the Tevatron beam structure. A new code packet is sent every $132 \mathrm{~ns}$. The framing bit is always set high and is monitored by a state machine to ensure that the link is synchronized. The crossing bit is only set if there is beam for that particular $132 \mathrm{~ns}$ period. The sixteen possible encoded data commands include commands such as Acquire, Preamp Reset, Level 1 Accept, Digitize, Readout, and Readout Abort.

The NRZ link has an adjustable delay system to synchronize the crossing command with actual beam crossings so that SVXIIe charge acquisition happens at the optimal time with respect to beam. There are three delays, $132 \mathrm{~ns}$, $18.8 \mathrm{~ns}$, and $2 \mathrm{~ns}$ steps, writable from a MIL-STD-1553 serial communication link. This MIL-STD-1553 link is also used to set various diagnostic modes.

To prevent missed triggers, the SEQC sends a Busy signal back to the Trigger Framework based on a logical OR of busy signals from the sequencers. This is necessary because the SVXIIe chips may operate in sparsification mode, reading out only the channels over threshold, and readout may end at various times after a trigger. Since there is only one buffer on the SVXIIe, a new trigger may be accepted only after readout has finished.

A Diagnostic mode is provided to exercise the data acquisition system if the Trigger Framework signals are not operational. This mode is exercised via MIL-STD-1553 link.

\subsection{SVXIIe Sequencer}

The basic task of the SVXIIe sequencers is to coordinate data acquisition in the SVXIIe chips and serialize the resulting data onto high speed fibers to be sent to data storage.

The sequencers are $9 \mathrm{U} \times 340 \mathrm{~mm}$ circuit boards that reside in slots 2 through

2 The Trigger Framework gathers digital information from each of the specific Level 1 trigger devices and chooses whether a particular event is to be accepted for further examination. 
21 in six Eurocard crates on the detector platform. Geographic addressing is designed into the backplane for each slot for MIL-STD-1553 Remote Terminal identification. Each sequencer is connected to an interface board via four 50-conductor 3M pleated-foil cables, a VRB via four optical fibers for data readout, the SEQC via the backplane, and the control system via the MIL-STD-1553 link which is also plugged into the backplane.

In initialize mode, the sequencers interpret data from the MIL-STD-1553 data bus and then clock the appropriate download data pattern into the chips. This pattern is readable from the chips only by shifting new data into the chips so a simultaneous download of all chips on an HDI must occur. In acquire mode, the sequencers advance the pipeline clock with each beam crossing, and the SVXIIe chips' preamplifiers are reset during beam gaps. When a trigger occurs, a specific complex manipulation of the control signals occurs which extracts charge out of the correct pipeline cell, and then the sequencer sends a $53 \mathrm{MHz}$ clock which the chip uses to digitize this charge for each channel. In readout mode, clocks are sent to the SVXIIe chip at $26.5 \mathrm{MHz}$, and the chip then sends alternately channel ID and data back to the sequencer. The sequencer serializes this data into a $1.062 \mathrm{~Gb} / \mathrm{s}$ data stream, adds header and trailer information, and sends it via optical fiber to the VRBs in the counting house.

Diagnostic features are interfaced to the MIL-STD-1553 bus and include a snapshot register to read the current state of important SVXIIe control lines and a built-in logic analyzer that records the same control lines for about $75 \mu \mathrm{s}$ after a selectable trigger. A pattern of 64 words may be written via the MILSTD-1553 link and sent to the VRBs for testing the gigabit links. The Finisar laser [12] drivers' diagnostic links may be read via MIL-STD-1553, monitoring power output, temperature, and other parameters. Other MIL-STD-1553 link registers include SVXIIe chip power on/off control, module serial number, and a remote programming register.

An optional Readout Abort feature is used to guard against system hangups from non-responding chips. Normally the sequencers use the Priority_Out handshake signal from the last chip in a chain to determine when readout is finished. If this handshake ever fails, a $45 \mu$ s timeout in the SEQC propagates to the sequencers and puts the system back in acquire mode, and Busy is released.

\subsection{VME Readout Buffer}

The VME Readout Buffer (VRB) is a $9 \mathrm{U} \times 400 \mathrm{~mm}$ multiport memory that buffers the data for transfer to the higher level data acquisition system. It 
contains ten independent input ports and a common VME output port. For SMT operation, eight channels on each board are used.

The VRB acts as a buffer as data wait for the conclusion from the second decision level (Level 2) of the hardware trigger system [4]. Buffer management is provided by an external System Controller (VRBC) through a dedicated control port. A controller serves up to $12 \mathrm{VRB}$ modules in one VME crate.

The VRB receives data via serial optical connections from the sequencers via the VTM which converts the optical signal to an electrical signal. Input data is accepted at an aggregate rate of approximately 500 MBytes/s on eight (bytewide) channels. The output rate is limited by VME transfer speeds and by the number of VRB modules sharing the VME bus. For SVXIIe chip applications, the VRB inputs data at the accept rate of a few $\mathrm{kHz}$ from the first decision level (Level 1) of the hardware trigger, and outputs data at the Level 2 accept rate of less than $1 \mathrm{kHz}$.

The VRB Control Logic performs three basic functions: receive and process messages from the system controller, return status signals to the system controller, and manage the general flow of data to and from the VRB buffers. The Control Logic will respond to a message by asserting a status signal, typically within $200 \mathrm{~ns}$.

Upon a Level 1 trigger accept, the VRBC supplies the VRB with a buffer number to store the input data. When the VRB Control Logic receives a message specifying the next input buffer, it looks up the buffer starting address in the shared memory, and broadcasts this information to the Receive Logic for all channels. The input data is pushed to the VRB from the sequencer. The event data from an SVXIIe chip is logically organized by words ( 2 bytes). The first bytes of each data stream contain a header inserted by the sequencer to identify the data source. This is followed by a block of data from each SVXIIe chip containing the chip ID, status, and up to 128 pairs of channel number and data. The VRB input FIFO (First In First Out memeory configuration) will accept data until it recognizes an End Of Record word. When all event data is received, the VRB Control Logic will read the individual byte counts from each Receive Logic block and generate a global byte count for the event. The global byte count is available to a VME Scan Processor so that it can perform a single block read operation to obtain all data for the event. When all the channels transmitting data to a VRB are done, the VRB will inform the VRBC. This is accomplished by releasing a busy signal on a dedicated line of control bus. The busy line is an open collector signal that can be driven low by any of the VRBs in the crate. The transfer of the data from the SVXIIe to the Level 2 buffer is finished when all VRBs have released signal on this line.

Following a Level 2 Accept, the VRBC supplies the VRB with a buffer number 
for data output. Events that are accepted by the Level 2 trigger are copied to the VME output data FIFO. For events rejected by the Level 2 trigger, the VRBC re-uses the buffer number, causing the previous event data to be overwritten. 


\section{Silicon Sensors}

The SMT detector uses a combination of single-sided, double-sided, and doublesided double-metal silicon sensors.

Single-sided and double-sided devices were produced from high resistivity 4" silicon wafers with crystal orientation $<111>$ and $<100>$. The double-sided double-metal detectors were manufactured using $<100>6$ " wafers. Isolation on the n-side of all double-sided devices is provided by p-stop implants. AC coupling aluminization was specified to be $2 \mu \mathrm{m}$ inside the strip to limit edge fields which might cause micro-discharge breakdown. All traces are biased using poly-silicon resistors.

Of particular importance for the double-sided devices is the quality and robustness of the AC coupling capacitors, since these are required to isolate the readout electronics from the detector depletion voltage. To minimize the stress on these capacitors, the detectors are in general operated with the bias split between the n- and p-sides. The voltage ratio is limited by the micro-discharge effect discussed in Sec. 5.4. Table 8 summarizes the sensor locations and types, and Fig. 17 summarizes the geometry of the individual detectors.

Table 8

Characteristics and deployment of various sensor types. The length of the inner $\mathrm{H}$-disk sensor is indicated with $i$ and the outer with $o$.

\begin{tabular}{|c|c|c|c|c|c|c|}
\hline Module & Type & $\begin{array}{c}\text { Pitch } \\
\text { p-/n-side } \\
{[\mu \mathbf{m}]}\end{array}$ & $\begin{array}{c}\text { Length } \\
{[\mathrm{cm}]}\end{array}$ & $\begin{array}{c}\text { Inner } \\
\text { Radius } \\
\text { [cm] }\end{array}$ & $\begin{array}{c}\text { Outer } \\
\text { Radius } \\
{[\mathrm{cm}]}\end{array}$ & Manufacturer \\
\hline F-disk & DS & $50 / 62.5$ & 7.93 & 2.57 & 9.96 & Micron Eurisys \\
\hline H-disk & SS & $\begin{array}{c}40 \\
80 \text { readout }\end{array}$ & $7.63^{i}, 6.39^{o}$ & 9.61 & 23.6 & Elma \\
\hline Central & DSDM & $50 / 153.5$ & 12.0 & 2.72 & 7.58 & Micron \\
\hline Barrels & DS & $50 / 62.5$ & 6.0 & 4.55 & 10.51 & Micron \\
\hline Outer & SS & 50 & 6.0 & 2.715 & 7.582 & Micron \\
\hline Barrels & DS & $50 / 62.5$ & 6.0 & 4.55 & 10.51 & Micron \\
\hline
\end{tabular}




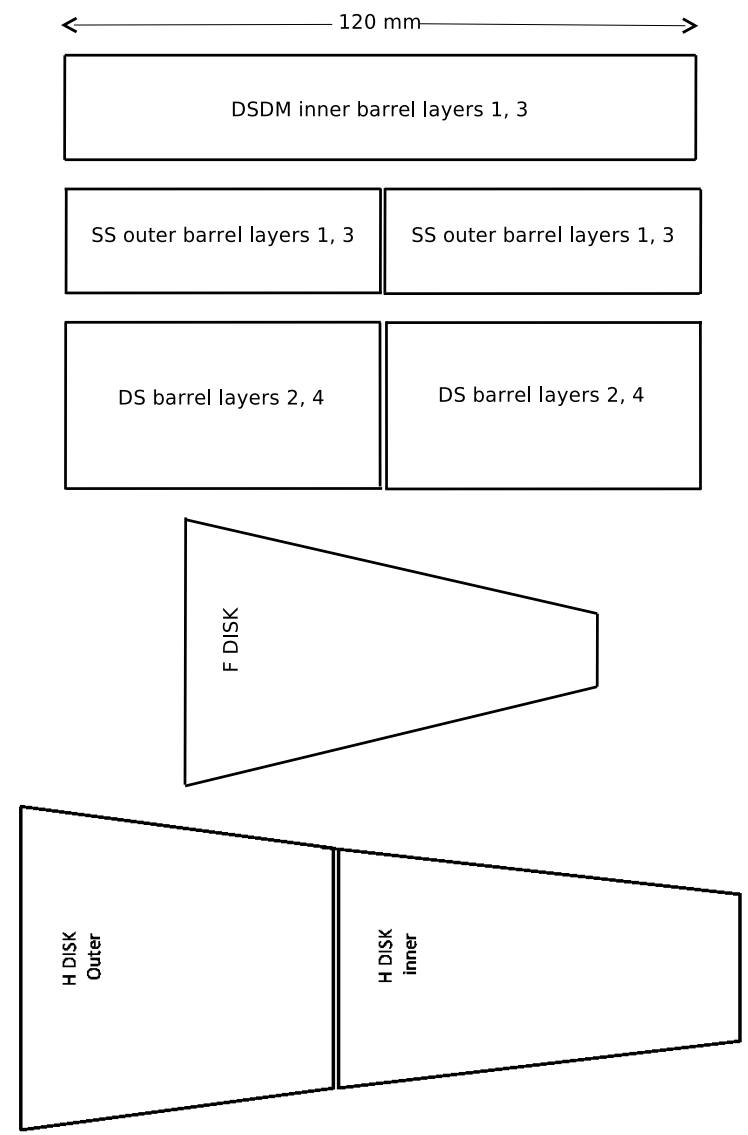

Fig. 17. The geometry of all individual detectors showing their relative dimensions.

\subsection{Barrel Sensors}

Three types of sensors are used in the central barrels. The second and fourth layers use double-sided stereo detectors with the n-side implants at a $2^{\circ}$ angle with respect to the p-side axial strips. Two of these sensors are bonded together to form a $12 \mathrm{~cm}$ long ladder.

On the first and third layers of the outer barrels, single-sided sensors with axial strips are used. Also in this case two sensors are bonded together to form a $12 \mathrm{~cm}$ long ladder. The inner four barrels use $90^{\circ}$ stereo sensors. Ninety degree readout was achieved using a second metal layer on the n-side insulated from the first metal layer by $3 \mu \mathrm{m}$ of PECVD (Plasma Enhanced Chemical Vapor Deposition) silicon oxide. Two readout strips on this side are multiplexed to a single readout channel. Implants on the n-side are isolated by individual p-stop frames in addition to a common p-stop enclosure. The use of 6 " silicon 
wafers allowed production of $12 \mathrm{~cm}$ long DSDM sensors as single pieces.

\subsection{Disk Sensors}

The disk sensors are trapezoids with readout strips arranged parallel to the long edge of the devices. In this arrangement the strip length varies for strips which originate past the base of the trapezoid.

The F-disk detectors provide $\pm 30^{\circ}$ stereo point measurements which provide good impact parameter resolution in both $r-\phi$ and $r-z$.

For the H-disks, two detectors are bonded together to form the readout module. The strips of the inner and outer sensors are bonded together, leading to a maximum strip length of $14.24 \mathrm{~cm}$. The single-sided modules are assembled back-to-back on a beryllium substrate forming a $15^{\circ}$ stereo angle.

In both detector types, a small region in the upper corner of the device, where strip lengths would be less than about $1 \mathrm{~cm}$, is left unbonded.

\subsection{Sensor Production and Testing}

The sensors were produced by three different vendors, Micron Semiconductor LTD [13], Elma [14, and Canberra Eurisys Mesures [15]. Those produced by Micron were tested at the Micron factory by D0 personnel; Elma sensors were tested at Moscow State University; and Eurisys sensors were tested by the company. The bulk of the sensors used in the experiment were produced by Micron and the testing of these sensors is described in more detail below. Table 9 summarizes the specifications for the Micron devices.

One of the most important electrical characteristics of the silicon sensors is the depletion voltage. It was estimated by studying the capacitance-voltage relationship measured at a dedicated station at Micron. A typical measurement is shown in Fig. 18 where the inverse of the capacitance squared is plotted as a function of bias voltage. The depletion voltage is given by the intersection of the plateau, seen in figure, with the $1 / C^{2}$ slope.

Other basic detector tests included:

- measurement of IV characteristics and breakdown voltage

- measurement of AC coupling capacitor value

- measurement of AC coupling capacitor leakage and pinholes 3 to $100 \mathrm{~V}$.

$\overline{3}$ Broken AC coupling capacitors caused by flaws in the lithography could cause 
Table 9

Sensor specifications for the double-sided sensors fabricated by Micron.

\begin{tabular}{|c|c|c|}
\hline Parameter & F-disks and DS sensors & DSDM sensors \\
\hline$U_{\text {depletion }}(\mathrm{V})$ & $20<U_{\text {depletion }}<60$ & $20<U_{\text {depletion }}<60$ \\
\hline Total $I_{\text {leakage }} @ U_{\text {depletion }}+20 \mathrm{~V}$ & $<10 \mu \mathrm{A}$ & $<10 \mu \mathrm{A}$ \\
\hline$U_{\text {breakdown }}$ defined as $I=15 \mu \mathrm{A}$ & $>100 \mathrm{~V}$ & $>100 \mathrm{~V}$ \\
\hline$C_{\text {coupling }}$ & $>15 \mathrm{pF} / \mathrm{cm}$ & $>15 \mathrm{pF} / \mathrm{cm}$ \\
\hline p-side $C_{\text {coupling failures }}$ & $<2 \% / \mathrm{side}$ & $<4 \% /$ side \\
\hline n-side $C_{\text {coupling failures }}$ & $<2 \% / \mathrm{side}$ & $\pm 25 \%$ \\
\hline$R_{\text {bias }}$ & $1 \mathrm{M} \Omega<R_{\text {bias }}<10 \mathrm{M} \Omega$ & $1 \mathrm{M} \Omega<R_{\text {bias }}<10 \mathrm{M} \Omega$ \\
\hline$R_{\text {bias }}$ uniformity & $\pm 25 \%$ & $>100 \mathrm{M} \Omega$ \\
\hline$R_{\text {interstrip }}$ & $>100 \mathrm{M} \Omega$ & \pm \\
\hline
\end{tabular}

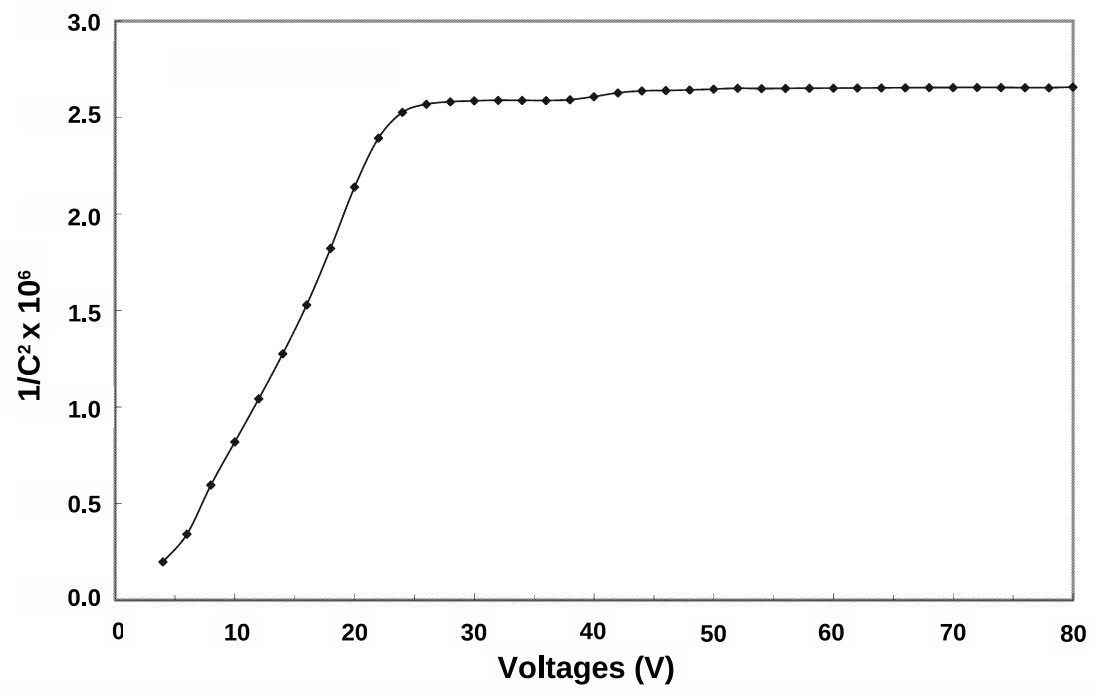

Fig. 18. $1 / C^{2}$ as a function of the bias voltage for a 9-chip sensor.

Figure 19 shows the yield of good strips for accepted sensors for DS ladders and F-wedges.

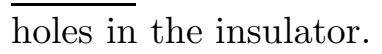




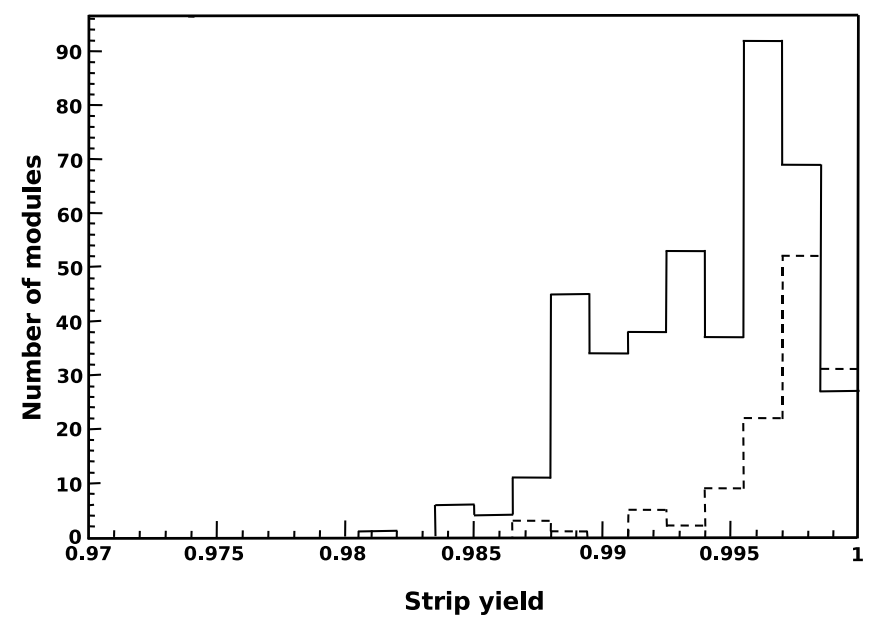

Fig. 19. The yield of good strips for accepted detectors for DS ladders (solid line) and F-wedges (dashed line).

Several problems were encountered during production which necessitated more detailed testing. Several devices showed regions of low interstrip resistance. This seemed to depend not only on device processing but also post-processing handling. These areas could be detected by current and resistance measurements on the DC contacts of the individual strips, and these tests were instituted for all detectors. Several detectors exhibited high leakage currents after the AC coupling capacitor tests. These detectors were cleaned and retested and accepted if the currents returned to previous levels.

Early in the production phase of the double-metal detectors some sensors were found with regions which exhibited large noise and high strip currents. By visual inspection, it was found that these regions were associated with flaws in the island p-stops on the n-side. These were traced to areas where the p-stop isolation implants were contacting the $\mathrm{n}^{+}$strip-defining implants with no intervening bulk material. In-process testing of n-side strips at Micron was introduced to identify these problems at an early stage of the processing. A strip current limit of $1 \mu \mathrm{A}$ at $80 \mathrm{~V}$ was used to identify p-stop problems and no detectors were accepted with more than one such flaw.

\subsection{Micro-discharge}

During the initial production testing, a number of devices were found that exhibited breakdown which depended on the voltage applied to the p-side of the device. The breakdown was due to "micro-discharge" [16]: avalanche breakdown of the $\mathrm{p}-\mathrm{n}$ junction when the potential between the negatively 


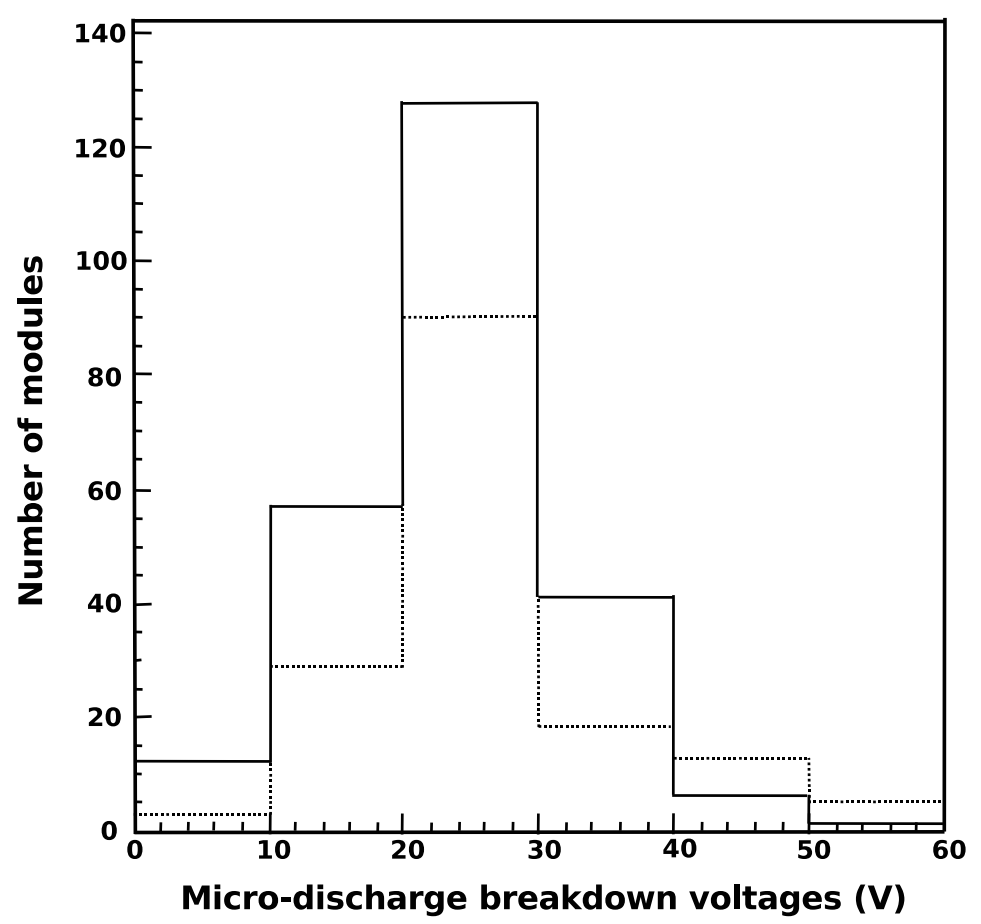

Fig. 20. N-side micro-discharge breakdown threshold for barrel layer DSDM detectors (solid line) and F-disk detectors (dashed line).

biased p-implant and grounded AC pad increases the junction field. This was confirmed by measuring the temperature dependence of the associated current, which increases with decreasing temperature due to the increase in carrier mobility. The threshold for this breakdown varied from detector to detector and was worst for those devices which had obviously misaligned implants and AC coupling strips. The threshold for the breakdown was affected by the amount of fixed positive oxide charge which increases the effective field near the $\mathrm{p}-\mathrm{n}$ junction. The existence of this breakdown made it necessary to characterize each device by the maximum p-side voltage which could be sustained without breakdown, as illustrated in Fig. 20, The detectors were then biased asymmetrically depending on this voltage.

Bare sensors were tested at the Fermilab booster irradiation facility $(8 \mathrm{GeV}$ protons) and the neutron irradiation facility at Lowell, MA, USA (one MeV neutrons). In addition, ladders were tested to $2 \mathrm{Mrad}$ in the Fermilab booster. In general the sensors behaved as expected from the Hamburg model [17] of reverse annealing and with a current damage constant of $\alpha=3.3 \times 10^{17} \mathrm{~A} / \mathrm{cm}^{2}$. The one exception to this behavior was the DSDM detectors, which exhibit anomalously rapid increase in depletion voltage with respect to the $2^{\circ}$ and disk detectors as can be seen in Fig. 21. Diode test structures from the same 6" wafers were also irradiated in the Fermilab booster. The behavior of these test structures was consistent with that of the detectors without double met- 


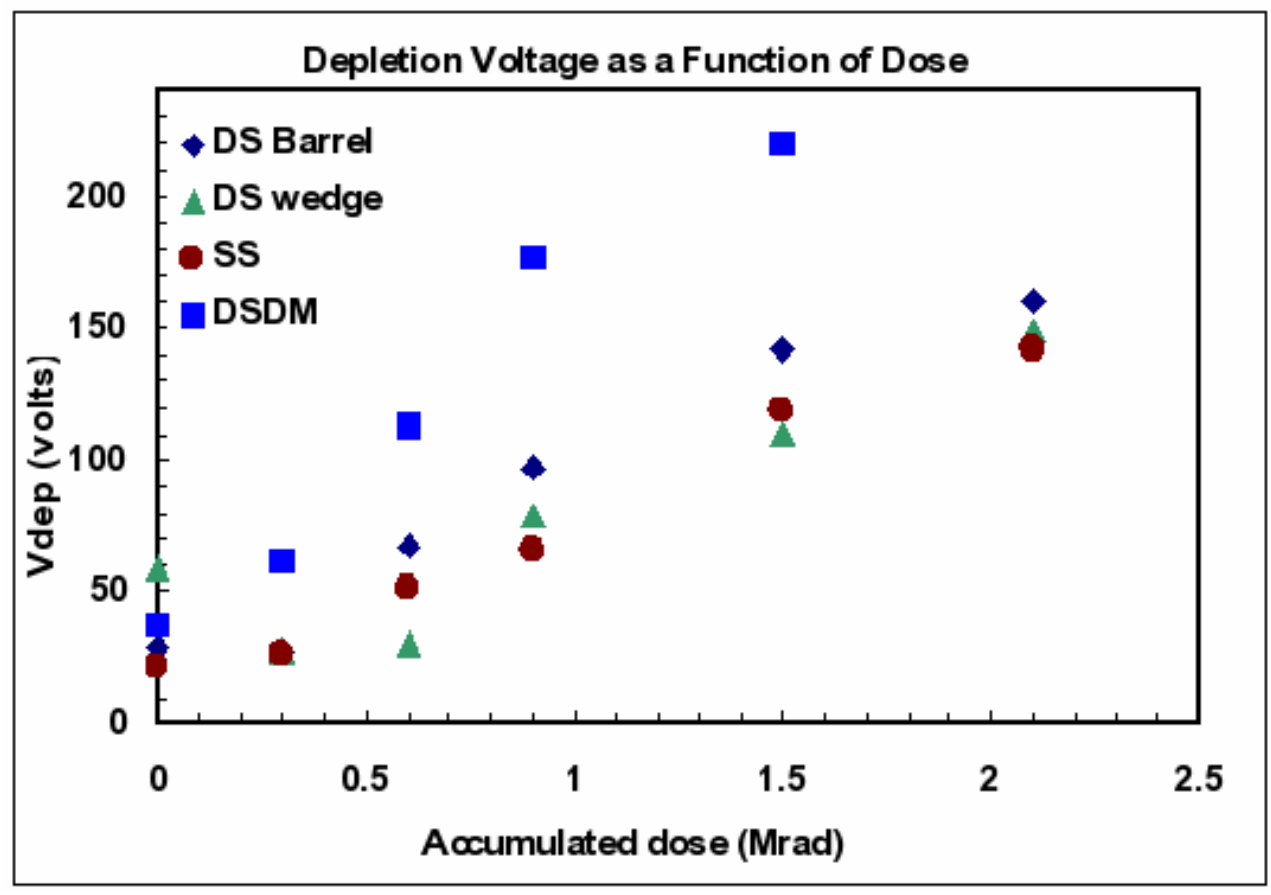

Fig. 21. Depletion voltage as a function of dose.

alization, indicating that the anomalous behavior is likely associated with the PECVD layer utilized in the double metal process.

The onset of micro-discharge will likely limit the lifetime of the SMT. After type inversion, the breakdown moves with the junction from the $\mathrm{p}$ - to the $\mathrm{n}$ side of the sensors. For a junction on the n-side, trapped positive charge in the oxide tends to reduce the field at the junction and the onset voltage of microdischarge is consequently higher on the n-side of an irradiated device than on the p-side of the same detector before inversion. Figure 22 shows the noise associated with the onset of micro-discharge after irradiation. Micro-discharge will limit the practical operating voltage of the DSDM detectors to about 75 to $100 \mathrm{~V} /$ side.

An assessment of the radiation damage with an estimate of the expected lifetime is described in Sec. 10 . 


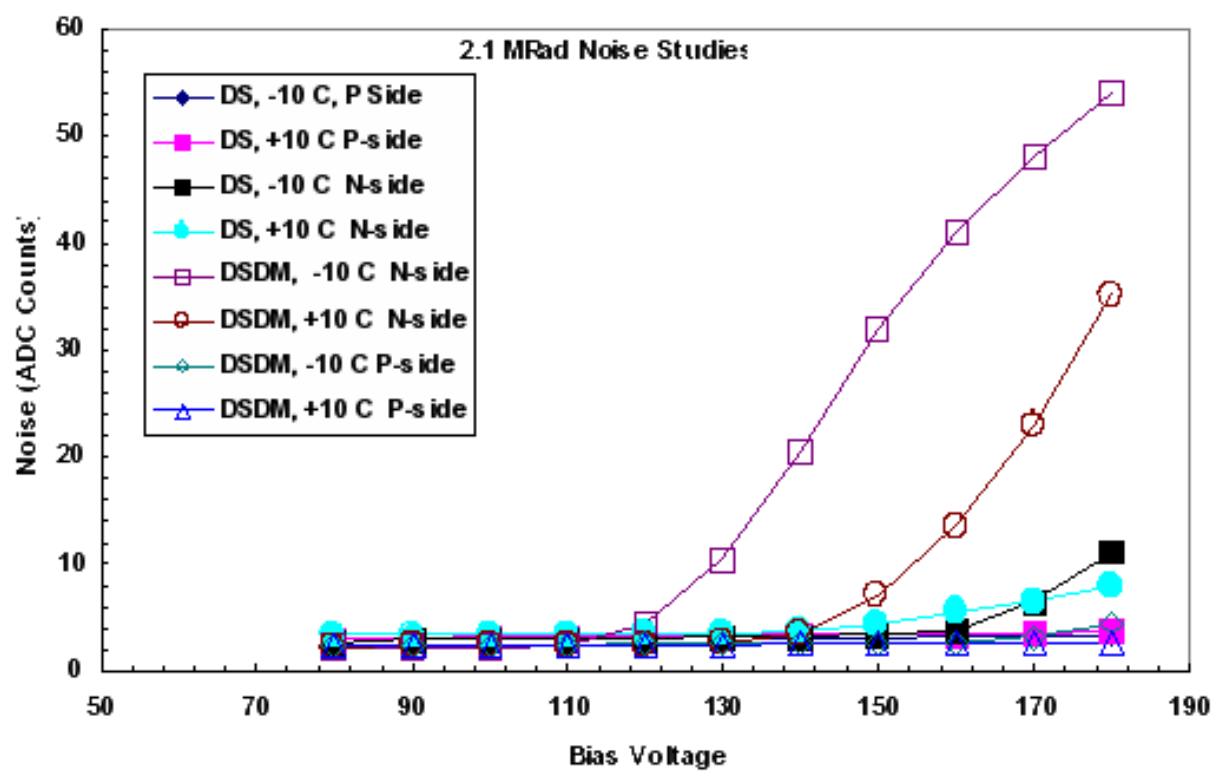

Fig. 22. Noise as a function of bias voltage at different temperatures.

\section{Production of Ladders and Wedges}

The large scale production of silicon detectors was a challenge for the D0 collaboration. A total of 1392 assemblies, which came in five different types, were required for building the SMT. In addition, the need for left and righthanded species of all the ladders also increased the complexity of the production work. This number excludes the up to $20 \%$ spare modules which were built. The large number turned the module assembly into a mass production which only functioned smoothly because of well defined procedures for the assembly processes.

All ladder and wedge modules were produced at the Silicon Detector Facility (SiDet) at Fermilab. This facility provided large clean room areas equipped with optical Coordinate Measurement Machines (CMM) and automatic bonding machines.

\subsection{Production of 3-, 6-, and 9-chip Ladders}

Since the basic layouts of the barrel ladders are very similar, these various ladder assemblies exploited almost identical techniques. The 3-chip ladders consist of two single sided sensors with an on-board mounted HDI for three readout chips. Their production was a one-stage process and therefore rather straightforward, so this assembly will not be described here. The 6- and 9- 


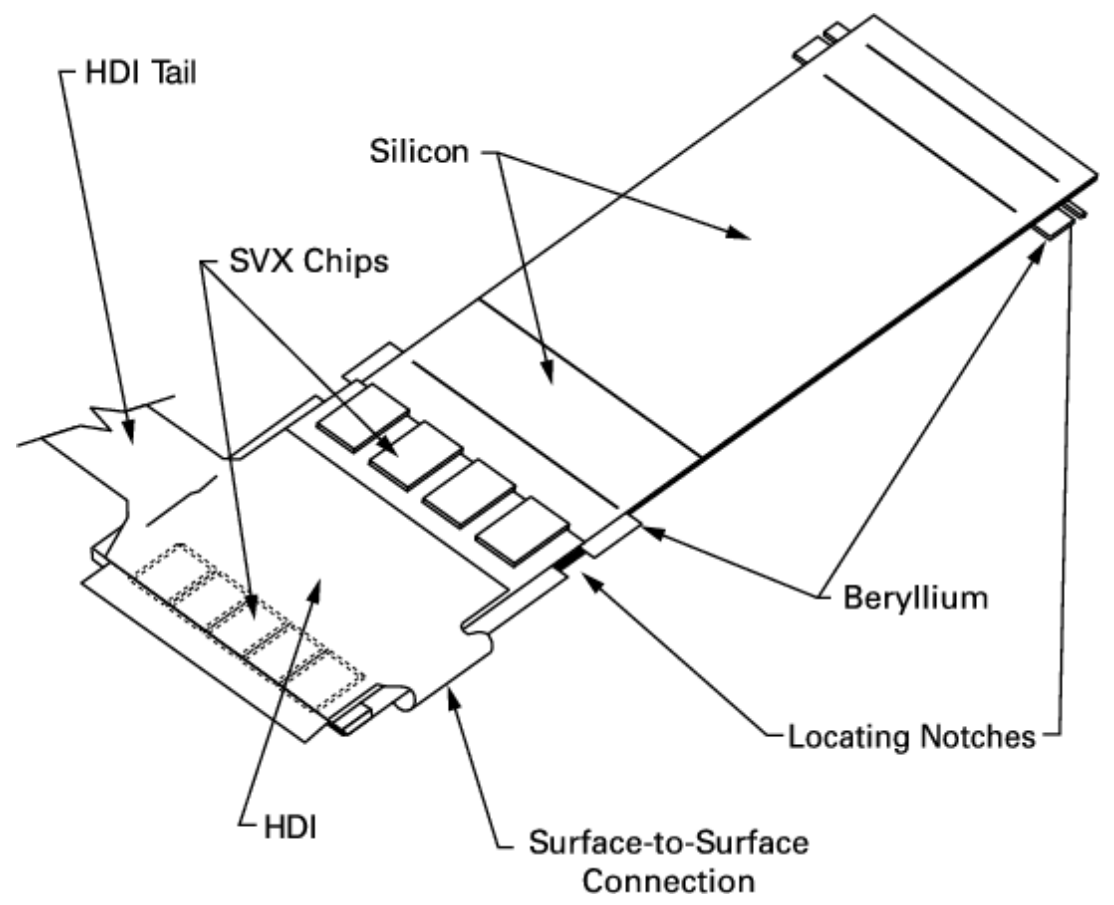

Fig. 23. Schematic design of a double-sided silicon ladder, isometric view.

chip ladders however, employed double-sided silicon which made the use of a two-stage production process with special fixturing for fold- and flip-over necessary. In the following sections, the successful two-stage production assembly is described.

\subsubsection{The Generic Double-sided Ladder Layout of the SMT Barrel Detectors}

The basic design of the 9-chip double-sided ladders is shown in Fig. 23. The isometric drawing shows a 9-chip ladder with 5 and 4 readout chips on the pand n-side respectively. The two silicon sensors are ganged by wire-bonds and supported by carbon fiber rails. The HDI, laminated on the beryllium pieces prior to ladder assembly, was wrapped around the two surfaces of the silicon and connected to the silicon strips on both sides of the sensors. Figure 24 shows an elevation view of the on-board mounted readout assembly.

\subsubsection{The Assembly Components and Assembly Preparations}

Sensors used for ladder production were selected according to the results from the measurements described in Sec. 5. In the case of 3- and 9-chip ladders, which consist of two sensors, the depletion voltage of the individual sensors was matched.

The selected sensors were visually inspected to ensure a clean surface on the bonding pads prior to the assembly process. Spots of organic residue on sen- 


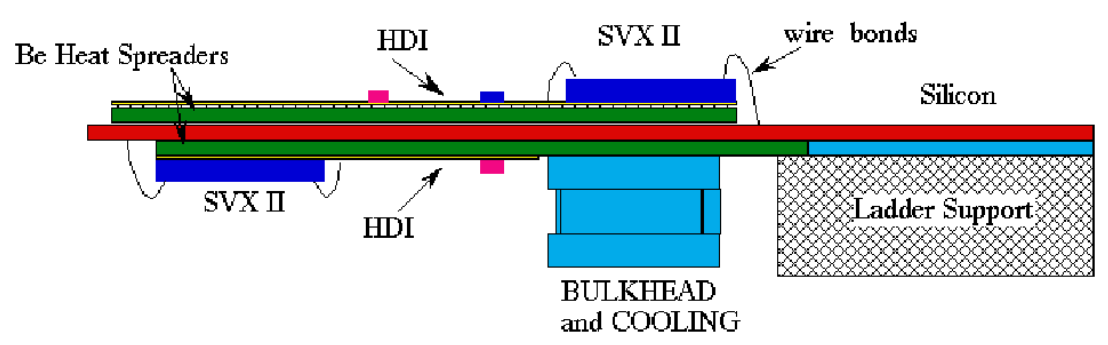

Silicon Readout Assembly

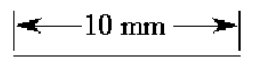

Vertical scale $\mathrm{x} 2$

Fig. 24. Schematic design of a double-sided silicon ladder, elevation view. Note that the vertical size is not drawn to scale.

sors, in particular on the AC-bonding pads, caused a few unreliable bonds.

Beryllium was chosen as the heat spreader material due to its excellent thermal and electrical properties, its high specific stiffness, and its very long radiation length. The beryllium pieces used in the ladder design were machined into their final shapes by the supplier [18], so that no further machining was necessary at Fermilab.

Three different beryllium substrates were used in the assembly process of the ladders. The "active cooled" and "passive" beryllium pieces were positioned on the p-side of the sensor, whereas the beryllium "top plate" was located on the n-side. The typical thickness of the beryllium substrates is $400 \mu \mathrm{m}$ for the ladders. The beryllium pieces aid in stiffening the ladder, provide an improved thermal path, and serve handling and reference purposes. The active and the passive beryllium pieces were used for the alignment. They have precisely machined notches at the side (machining accuracy of notch surface $\pm 5 \mu \mathrm{m}$ ) in order to locate a ladder into a barrel by press fitting one of the notches of each beryllium piece into machined posts at the bulkhead surface. All beryllium pieces were visually inspected prior to lamination and the specified mechanical dimensions measured. The thickness was determined at five different points. The $50 \mu \mathrm{m}$ flatness specification of the substrates was verified, and the upper tolerance of the notch-notch distance was checked with each beryllium piece on a small fixture.

The HDIs were laminated to the active and top plate beryllium pieces by using a $25 \mu \mathrm{m}$ thick film and a $75 \mu \mathrm{m}$ thick adhesive film [19]. An additional thermally and electrically conductive path through silver epoxy loaded holes in the HDI was provided so that the beryllium pieces could remove the heat load of the SVXIIe chips and be kept on a common system ground.

The beryllium substrates also help to maintain the flatness of the HDI. The two beryllium (active and passive) pieces with the locating notches were con- 
nected by two support rails, each consisting of a carbon/boron fiber, Rohacell foam, carbon/boron fiber sandwich. These rails were glued to the silicon ladder parallel to the long side in order to make the ladder stable and stiff. The carbon/boron fiber composite was designed to match the thermal expansion coefficient of the silicon. The carbon fiber support rails also serve as low impedance connections in order to ground the passive beryllium piece at the ladder's end.

For each 3-, 6-, or 9-chip ladder, there were two basic ladder types produced: left-handed $(\mathrm{LH})$ and right-handed $(\mathrm{RH})$ ladders. Both types were produced in equal number. They differ by the relative orientation of the beryllium notches in the bulkhead posts with respect to the orientation of the ladder axis. 4 . Two different fixtures were employed for RH and LH ladders. These assembly fixtures were made out of either aluminum or steel and were coated with a layer of Teflon to allow a gentle sliding of the silicon sensors for accurate alignment.

The fixtures had pushers at the side to exactly place the silicon sensors with respect to the glass scale targets on the fixture. Additional steel posts had been inserted into the fixture in order to press fit the notches of the active and passive beryllium pieces against these posts. Spring loaded clamps on the fixture supported the press fit by pushing the beryllium notches against the posts. All the alignment and assembly of the silicon ladders was done under control of a CMM.

Before ladder production started, the final position of the glass scales and the steel inserts on the fixtures was determined in an iterative procedure. The qualification process included the assembly and measurements of test ladders as well as the fixture targets until the resulting distances between the notch edges of the beryllium and the silicon center axis defined by the silicon targets converged to within the desired specifications of $5 \mu \mathrm{m}$.

\subsubsection{Ladder Production}

Before the assembly started the components were tested. The testing procedure is discussed in Sec. 7.

The first stage of the assembly, Stage-I, of a 6- or 9-chip ladder was the alignment of the silicon sensor piece(s) on the assembly fixture under the control of an optical CMM. The axial strip side (p-side) of the silicon faced up. Once the silicon was aligned, the vacuum on the fixture was turned on to keep the sensors in position. The alignment was then verified with the CMM, and if

\footnotetext{
4 In addition, the LH and RH ladders have two different HDI tail lengths.
} 


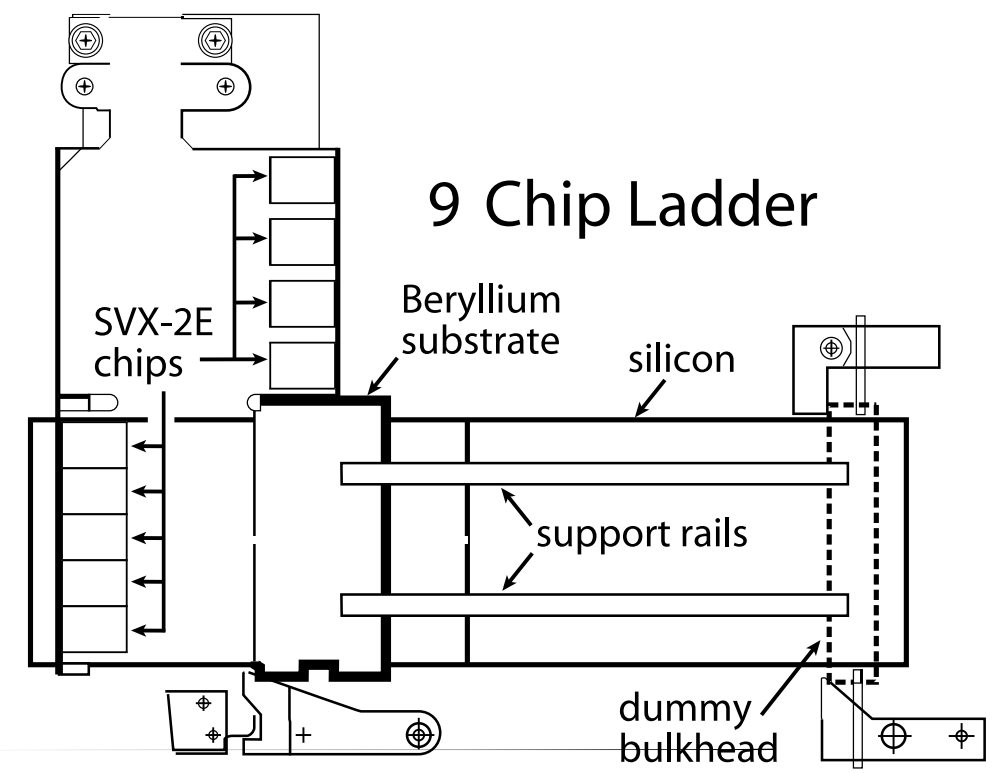

Fig. 25. Schematic drawing of a 9-chip ladder resting on the Stage-I assembly fixture.

needed, the sensors were readjusted in order to keep their position uncertainty below $5 \mu \mathrm{m}$.

HEXCEL epoxy glue was applied onto one film laminated beryllium carrier piece of the HDI and onto the smaller passive beryllium piece at the other end of the ladder. An exact amount of epoxy controlled by a glue dispenser was used and the glue was distributed in a fish-bone-like pattern on the beryllium pieces to ensure an evenly spread glue joint. The HDI with the laminated beryllium pieces was then glued onto the silicon surface. It was checked that the spring loaded pushers were pushing the notches of the active and passive beryllium pieces tightly against the steel posts in the fixture. Figure 25 shows a schematic drawing of a 9-chip ladder resting on the Stage-I fixture. Figure 26 is a photograph of a 9-chip ladder on that fixture.

After setting special gluing weights above the adhesive joints between HDI and silicon, the ladder alignment was rechecked with the CMM. The gluing weights were applied for at least twelve hours. The thickness of the glue joint after the epoxy is about $75 \mu \mathrm{m}$.

After curing of the glue, the sensor was bonded to the readout chips and - in case of the 3- and 9-chip ladders - sensor to sensor bonds were made using the same assembly fixture as a bonding jig. A visual inspection of the wire bonds followed after each bonding step. The carbon/boron fiber rails were then glued on the silicon ladder connecting the active and passive beryllium piece with a low impedance path. The rails were glued using the same HEXCEL epoxy onto the silicon sensors, whereas the adhesive joint to the beryllium plates was made by small amounts of conductive silver epoxy. 


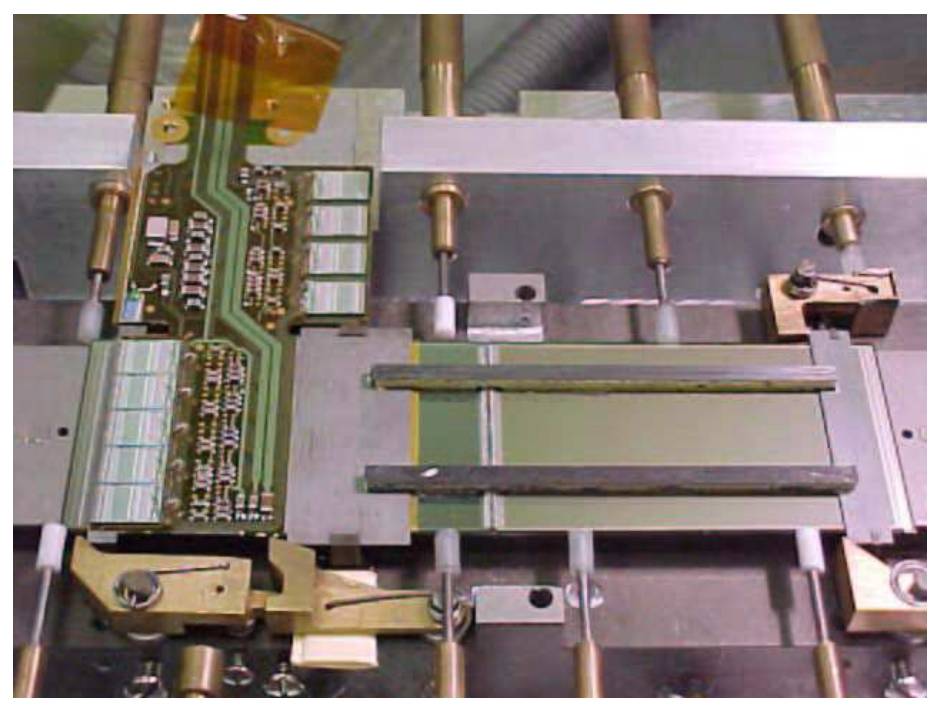

Fig. 26. A photograph of a 9-chip ladder on the Stage-I fixture.

In the second assembly stage, Stage-II, the ladder was flipped to the n-side using a special pickup fixture. The HDI was folded over and centered onto the n-side of the silicon sensor with the help of spring loaded pushers at the Stage-II fixture. The second part of the HDI serving the n-side was then glued with HEXCEL epoxy to the sensor, and another gluing weight was applied during the curing cycle. After this, the sensor to sensor bonds on the n-side and the bonds to the readout chips were done.

The production times of the ladders depended on the ladder type. The single sided 3-chip ladders could be produced within one day. The double-sided 6-chip ladders, having only one sensor, could be fabricated within two days, while almost three days were necessary for the production of double-sided 9-chip ladders due to the additional sensor to sensor bonds. While the individual production time of a ladder was mainly determined by the curing time of the glue, the overall pace of the ladder fabrication was dominated by the availability of the parts and components. Duplicate sets of assembly fixtures and up to four CMM machines with mounted fixtures were used to keep up with the tight production schedule. The number of assembled ladder modules versus time is shown in Fig. 27.

\subsubsection{Mechanical Accuracy of the Ladders}

Since the ladders were inserted into the bulkheads by pressing the notches of the active and passive beryllium pieces against the bulkhead posts, the final location of the ladder in the bulkhead and hence the axial alignment of the silicon strips are determined by these two notches. Any tilt between the two beryllium notches with respect to the silicon strips leads to a misalignment. The achieved mechanical accuracy in the ladder assembly is shown in Fig. 28. Figure 28a and b show the maximum tilt between the active and passive ends 


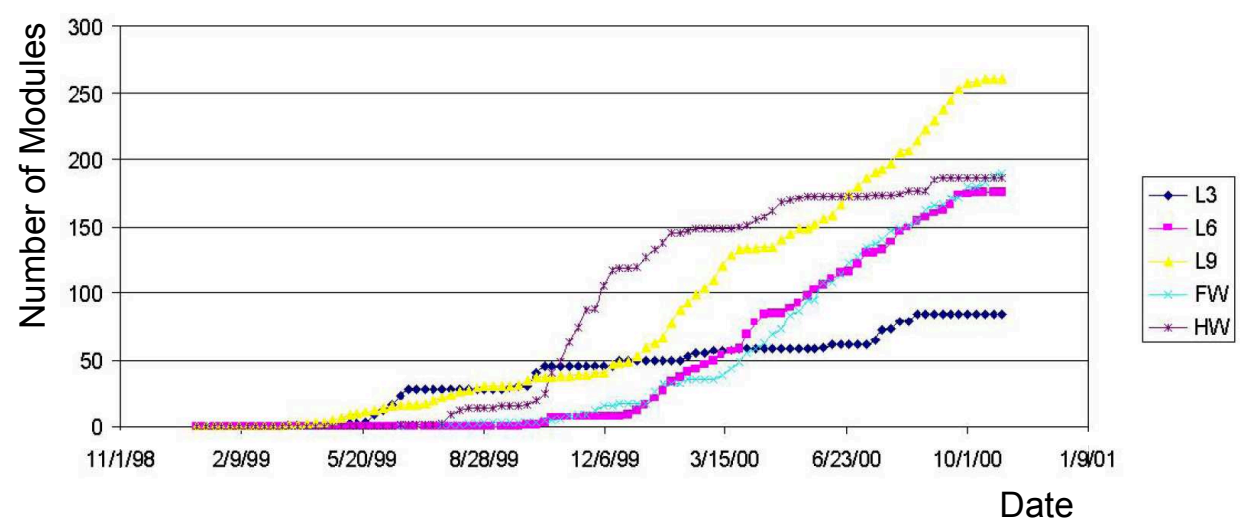

Fig. 27. Production of ladders and wedges as function of time. The overall production pace was mainly determined by the availability of the parts.
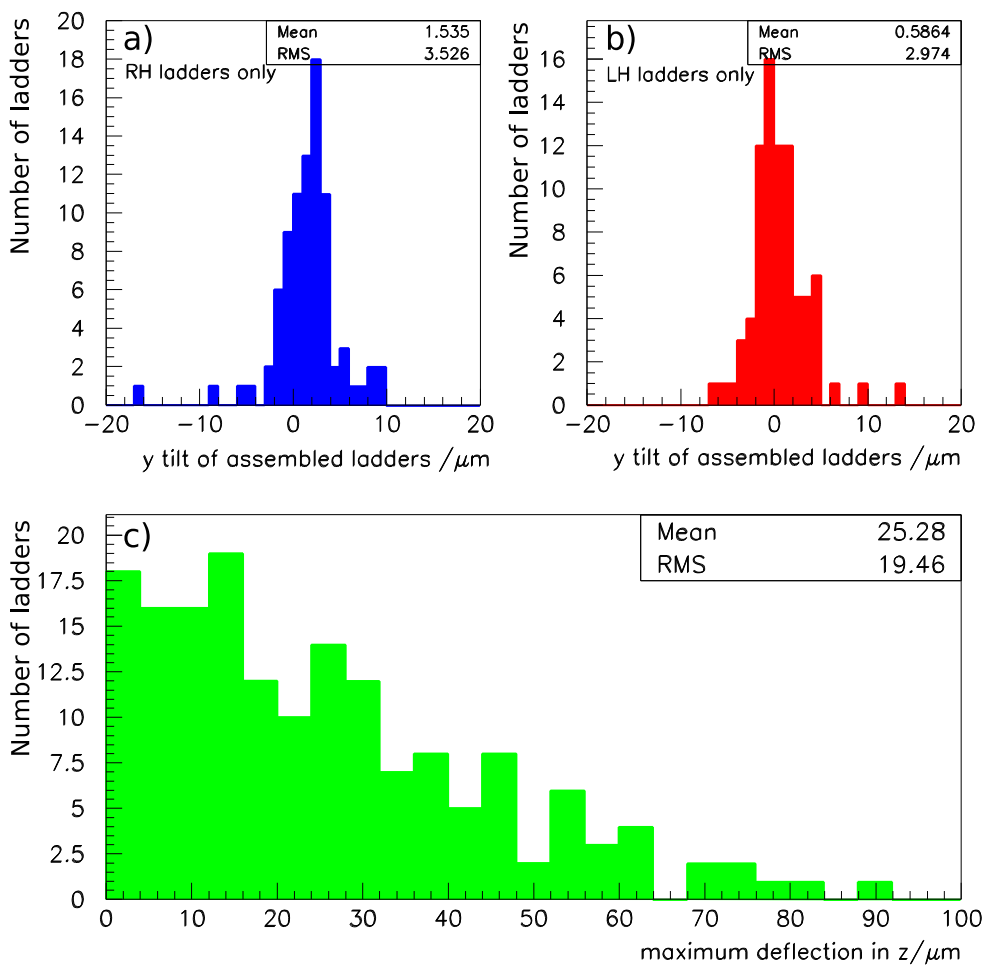

Fig. 28. Mechanical accuracy of 6-chip ladders for (a) RH ladders, (b) LH ladders, and (c) all ladders.

of the beryllium for all production 6-chip ladders on the LH and RH fixtures. The distributions are well centered and have an rms value of about $3.5 \mu \mathrm{m}$. Figure 28k shows the absolute value of the maximum deflection normal to the sensor plane of all produced 6-chip ladders. The flatness measurements were performed on an optical metrology machine. Almost all ladders are well below $50 \mu \mathrm{m}$, which is the maximum radial displacement permitted for the inner layer. 


\subsection{F-Disk Wedge Production}

\subsubsection{Gluing Process}

Qualified sensors and HDIs were selected to be glued together. The HDI was mounted on the same carrier bar that was used for testing and shipping. This bar was also used to help handle the HDI for the gluing procedure. There were two important considerations for the gluing process: a) relative alignment of the sensor to the HDI, and b) the thickness of the glue joint such that good contact was insured. The process was performed on a specially machined fixture that was mounted on a CMM.

To control the thickness of the glue, the thicknesses of the HDI substrate as well as the silicon sensor had to be known. The silicon sensor thickness as measured by the manufacturer was used but the HDI thickness was measured directly. Once the thicknesses were known, the correct thickness gauge blocks were chosen to mount on the fixture. The HDI was mounted on top of these gauge blocks in the fixture on the CMM. A coordinate system was defined from the HDIs fixed on the gluing fixture, with the $x(y)$ coordinate being in the direction of the base (length) of the wedge, and the $z$ coordinate perpendicular to the sensor surface.

The sensor was mounted on a flat pedestal and secured via a vacuum connection. This pedestal could be translated or rotated relative to the HDI using two micrometer adjustable screws. The plane height in the $z$ direction was fixed relative to the HDI. The alignment of the silicon relative to the HDI coordinate system was performed using two fiducial marks on the sensor. The first fiducial was in the center of the sensor right next to the HDI, the second was at the center-most part of the wedge as it would be placed on a disk. An iterative procedure was performed using the micrometer screws to allow both fiducials to line up at the correct places. This alignment was done to better than $5 \mu \mathrm{m}$ in the $x-y$ plane.

The same epoxy that was used for ladders was used to glue the wedges. The HDI was held down onto the silicon using spring loaded screws and left to cure overnight. After curing, the alignment was verified before the vacuum was released so that the new F-wedge could be dismounted from the gluing fixture and stored in its handling box. Because there are bias bonding pads on the very corner parts of the wedge, an extra step was taken to inject glue at these points. A trained technician using a small bore syringe performed this procedure.

A total of four gluing fixtures were made so that four F-wedges could be glued per day. The alignment and gluing procedure for these wedges could be completed in one hour during the afternoon. The subsequent corner gluing and 
visual inspection took another hour the following morning. Once the procedures were developed, there were very few problems during gluing. The main problem was assuring that the sensor did not come into contact with the alignment pin on the fixture during dismounting. The yield for this procedure over the entire production was over $90 \%$.

\subsubsection{Channel Bonding}

The F-wedges have a double-sided silicon sensor with one HDI with eight chips on one side and another HDI with six chips on the other side. On the side with six chips there was also a jumper, which is a pitch adapter to adapt from the chip spacing to the silicon spacing. Four production wire bonding operations were needed for each wedge. First the bonding from the chips to the sensors was done on the 8-chip side. Then the detector was turned over to the 6-chip side and the bondings from the chips to the jumper and from the jumper to the sensor were done. Finally the bias bonds to the detectors on both sides were attached. The total number of wire bonds for all wedges is about 370,000.

The 8-chip channel bonds were bonded using automatic wire bonding machines. This procedure took approximately 20 minutes per wedge. Only minor problems were encountered with this operation during development when the automatic bonder operating parameters were being determined.

The wedge-to-wedge height differences and non-similar jumper surfaces on the 6 -chip side made the wire bonding on the jumper side more problematic. Because of the awkwardness in bonding to the jumper, both of the bonding steps on the 6-chip side of the wedge were performed on semi-automatic bonding machines. The wedge was placed into a vacuum fixture for these steps. Bonding parameters occasionally had to be readjusted during this process, but the bonding was accomplished with very few unbondable channels. The two wire bonding steps on the 6-chip side took on average 45 minutes to one hour to complete.

The bias bonds are located on the corners of the sensor where the amount of glue underneath the sensor made the bonding difficult and therefore the bias bonds were made using a manual bonding machine.

Aside from the wire bonding technician, another technician helped to mount and dismount wedges from the fixtures and perform the quality assurance procedures. The majority of problems encountered were due to mishandling

of the wedges in the fixtures. Only one misalignment on the wire bonding machine broke a wedge. Only very minor problems were encountered with quality assurance of the bonding itself. These included tool marks and some wire bonds that broke after bonding. 


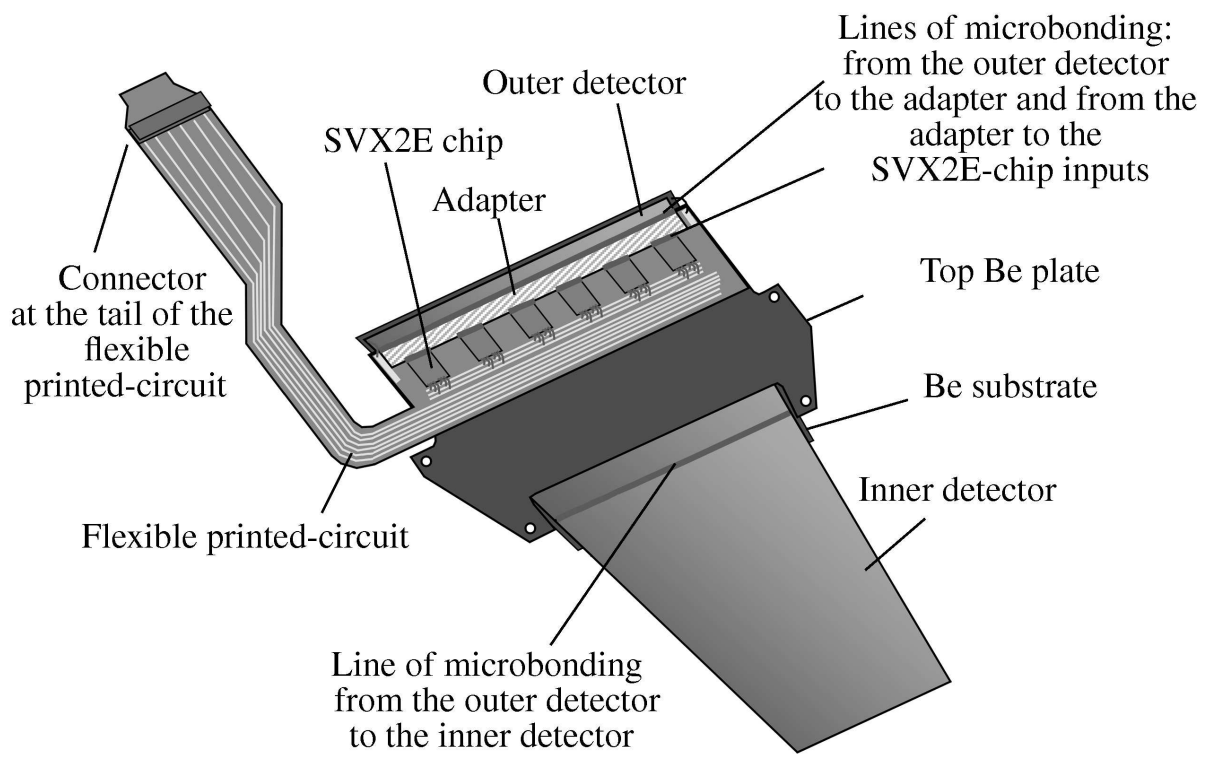

Fig. 29. Simplified configuration of the single-sided half wedge of an H-disk.

\subsection{H-Disk Wedge Production}

The H-disk wedges are two-layered and each wedge is composed of two singlesided half wedges which were glued back-to-back. A simplified configuration of the half wedge is shown in Fig. 29, Each half wedge consists of inner and outer silicon sensors mounted on a beryllium plate.

The single sided silicon sensors are shaped as trapezoids; their strips are parallel to one of the trapezoid's sides. The strips of the inner and outer sensors were bonded together, leading to a maximum strip length of $14.24 \mathrm{~cm}$. A beryllium plate was attached on top of the outer sensor providing a mounting surface for front end readout electronics installed on the HDI. The HDI was laminated in a similar way to the barrel ladders on top of the beryllium plate. The outer sensor strips were connected with the input pads of the chips with wire bonds. A special silicon based pitch adapter, fabricated from the same wafer as the wedge sensor, was used. A part of the top beryllium plate surface of one of the half-wedges was used for mounting of the wedge on the cooling ring of the disk. A full wedge module was produced by joining together two half wedges back-to-back.

\subsubsection{Wedge Production for the H-Disks}

Before assembly started, silicon sensors, beryllium substrates, and HDI flex circuits were thoroughly tested. Every step of the assembly process was followed by an electrical test of the subassembly. The half wedge assembly process 
started with lamination of the flex HDI circuits on the beryllium substrates. To adhere a large area of the outer silicon sensor to the beryllium plate, a thermal conductive tape based on an acrylic adhesive (3M9882) was used. This allowed stress relief on the silicon during assembly and cooling of the detector during operation. The substrates were aligned and bonded onto an outer silicon sensor using special assembly fixtures. To bond the inner silicon sensor to beryllium, the epoxy adhesive HEXCEL was used, the same type as was used in the ladder assembly process. The sensor alignment was done under CMM control. Wire bonding of the wedges was done in two steps. First, the SVXIIe chips were bonded to the pads on the HDI circuits. Second, the input pads of the chips were wire bonded to the strips from the outer sensor via a pitch adapter on the HDI, and the strips from the outer sensors were wire bonded to the corresponding strips of the inner sensor. All wire bonds were encapsulated on each of the half wedges to prevent damage during their assembly and installation on the mounting ring. A special bi-facial machine consisting of two co-axial microscopes was developed to align and bond together two half wedges back-to-back with precision better than $15 \mu \mathrm{m}$. The assembled wedges were installed on the beryllium cooling channel ring using the rotary table of a CMM with a precision of $25 \mu \mathrm{m}$. 


\section{Testing of Ladders and Wedges}

Thorough testing of each ladder and wedge at various stages of the production was required to avoid costly or even impossible back-tracking during the detector assembly. This section describes the procedures by which the modules were electrically tested during production and the criteria used in assigning an electrical grade to the finished detector modules. A more detailed description can be found in Ref. [20].

\subsection{Testing Sequence}

The essential building blocks of a detector module are the silicon sensors, the SVXIIe chips, and the HDIs. Each component was tested as described in Sec. 5, 7.2, and Sec. 7.3. The production testing sequence is described below, and can be summarized as follows:

(1) Bare HDIs that passed the functionality test at the manufacturer were sent to Fermilab for long-term testing.

(2) Sensors and burned-in HDIs were assembled into a detector module.

(3) Detector modules underwent initial functionality test.

(4) Detector modules that passed the initial functionality test were burnedin.

(5) Burned-in detector modules were laser tested.

(6) Detector modules were assigned an electrical grade.

(7) At each stage of the production sequence, malfunctioning modules were sent to be repaired.

Debugging and repair was done by expert physicists; the burn-in and laser tests were operated by non-expert physicists on shift with experts, who coordinated the activities and helped with the set-up of the tests. Detailed instructions of the daily activities and the procedures to be followed proved to be crucial to the operation, as was the meticulous bookkeeping of all activities. In the following sections, each step of the production testing sequence is described in detail. The overall yield of detector production was about $85 \%$. The lost detectors failed to pass either electrical or mechanical tests.

\subsection{Testing of the SVXIIe Chips}

The SVXIIe chips were produced on $12.7 \mathrm{~cm}$ wafers, with 148 usable dice on each wafer. A total of 206 wafers were produced and 155 of those were tested using an automatic probe station. One-hundred-twenty wafers were tested in 
the first half of 1997 (first batch), and 35 wafers were tested in the spring of 2000 (second batch). The testing and analysis procedures were identical for both groups of wafers.

The testing procedure consisted of driving all chip inputs with a computerspecified pattern, sampling the output lines, and checking that the output states were correct. The tests were separated into three categories: digital, analog, and miscellaneous. The digital tests exercised the readout and pipeline sections of the chip and checked that the chip current draw was within specifications for all supply voltages. The analog tests exercised the front-end preamplifiers and pipeline amplifiers, in both positive and negative modes. Pedestals and gains were measured for all 32 pipeline buckets for each of the 128 channels. Finally, the miscellaneous tests exercised all of the auxiliary registers, which control the calibration voltage, the ramp rate used for digitization, the input bandwidth, the pedestal level, and finally the chip bias current.

A probe station was used to physically position the wafer so that the probe card made contact with a specific die. One die was processed at a time; all the tests were run on that die, then the probe station moved the next die into contact with the probe card. Once the tests were completed, the wafer was diced and chips visually inspected for mechanical damage, such as scratched surfaces and missing corners. Two wafers were broken during the dicing process. 17593 chips (77\%) passed the digital tests, 14701 chips (64\%) passed the analog and miscellaneous tests, and 13988 chips (61\%) passed the visual inspection after dicing and were distributed. The yields from the first and second wafer batches were the same.

\subsection{Testing of Bare HDIs}

The HDIs are a two layer, $0.127 \mathrm{~mm}$ thick flex circuit of polyimide film with gold/nickel plated copper pads. The requirements of $0.127 \mathrm{~mm}$ wide pads and $0.051 \mathrm{~mm}$ wide via feedthroughs made the HDI a technical challenge for production. The tests checked for continuity and shorts between each of the pads on the front end and the 28 lines of the tail. This was accomplished through the use of a Ruker \& Kolls semi-automated wafer probe station, controlled through GPIB interface with a PC running LabView. Given a coordinate map of the HDI circuit, the probe station stage positioned each pad under a probe tip. Connection was made through a GPIB multiplexing box, and a GPIB controlled multimeter checked for continuity or shorts between the appropriate line on the tail and the probe.Approximately 60 pads were checked per SVXIIe chip mount point on the circuit.

The yields of good bare HDIs varied significantly between vendors and pro- 
duction batches. Litchfield Precision Components [21] had a very low yield of $\approx 30 \%$ after "fixing" (zapping the shorts with a high current). Dyconex [22] only delivered HDIs after internal testing, thus delivering good parts with excellent yield, but at a significantly higher price. A good compromise between cost and yield was finally reached with two additional vendors: Speedy [23] and Compunetics [24]. Obtaining working bare HDIs was one of the major issues of the early stages of the SMT production.

\section{$7.4 \quad$ PC Based Test Stands}

All the electrical tests performed on readout HDIs and detector modules used the same type of test stand, based on the Stand Alone Sequencer Board (SASeq), developed at Fermilab. The SASeq-based test stands were developed independently from the full D0 readout system. They were replicated and distributed at various locations at Fermilab and at remote institutes long before the final version of the full readout system was available. Using the PC-based version of the readout system proved crucial to the success of the production testing effort. The test stations used for the burn-in could operate 16 HDIs or detector modules at once. Separate one-SASeq test stands were also built which could read out two devices at once. The one-SASeq test stands were used for initial functionality tests of HDIs, debugging of detector modules, laser tests, repair, and for barrel/disk assembly. Nine stations were set up at the Fermilab Silicon Detector Facility, and three more stations were available at remote institutes.

The hardware configuration for the burn-in stations is shown in Fig. 30, It consisted of a VME crate that contained a Bit-3 VME controller card, eight SASeqs, a scanning 12-bit 64 channel analog-to-digital converter board (VME3113A) for current and temperature measurement, and a master vertical interconnect board for high voltage crate control. The two-channel SASeq board was a self-contained data acquisition card designed to interface to the SVXIIe chips. Its basic functions were to control the SVXIIe chip for data acquisition, collect the data when a data cycle was requested, and to relay the data to the processor in the crate. The crate also housed eight interface cards (IC). Each IC had two independent channels and was used as a bi-directional interconnect between the SASeq and the HDIs. The IC provided the control of the SVXIIe "power on" and "power off" sequence and prepared the monitoring of currents corresponding to the three SVXIIe operating voltages and to the temperature measurement.

Each SASeq was connected to an IC by a $3 \mathrm{~m}$ long, 50-conductor cable with an impedance of $82 \Omega$. Three low voltage power supplies were used to supply the three operating voltages needed by the SVXIIe chips. An SVXIIe voltage 


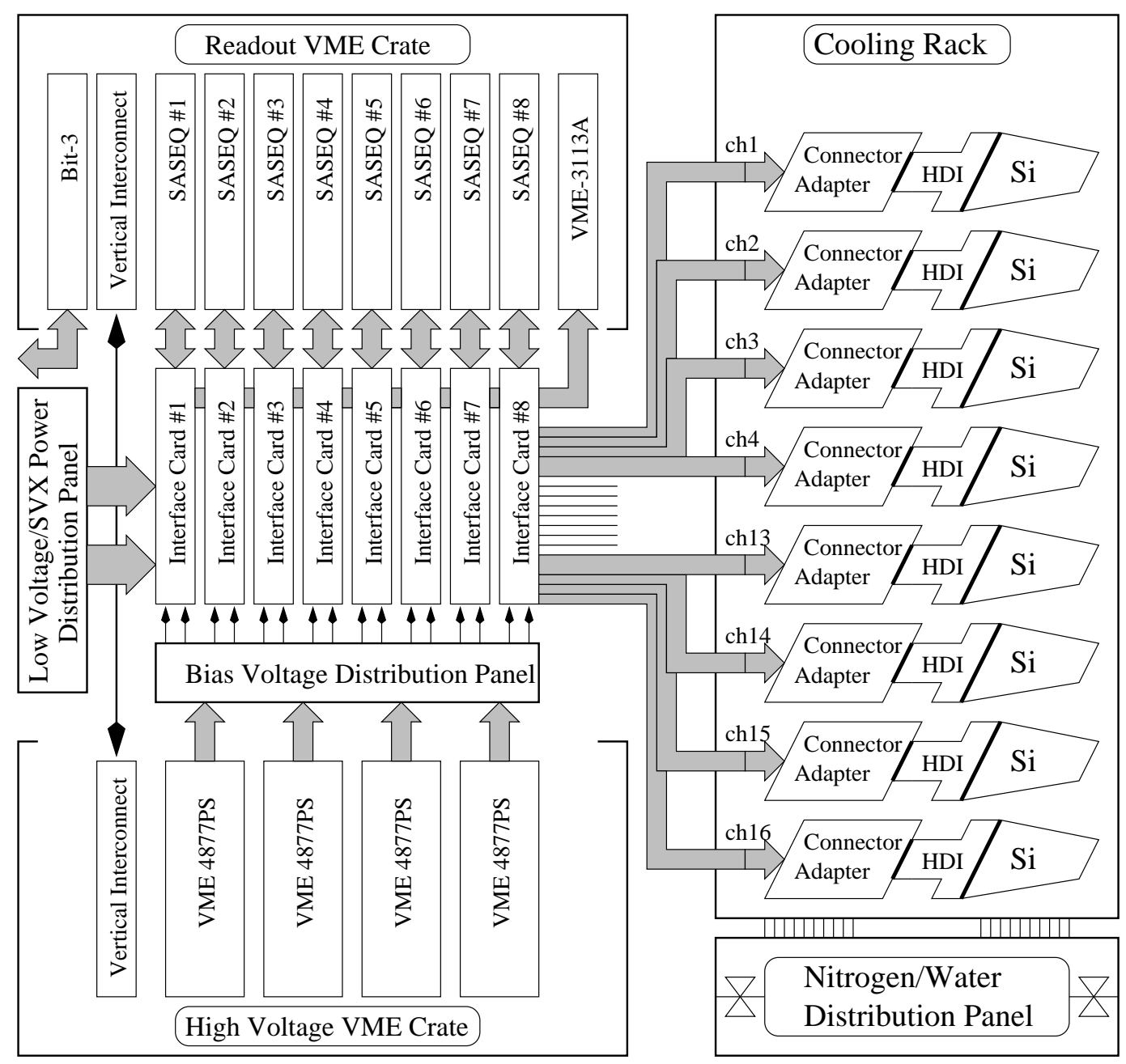

Fig. 30. Burn-in test setup. For debugging/repair/laser stations only one SASeq/IC pair was used, and no cooling was provided.

power distribution panel was located between the power sources and the IC crate and was used for the distribution of the SVXIIe power and the interface board power for each of the eight IC.

Bias voltage was provided independently for each detector module via a bias voltage distribution panel. The panel was located between the high voltage sources and the IC crate. A set of switches on the panel allowed for three different schemes for the biasing of double-sided detectors: positive bias applied to the n-side, negative bias applied to the p-side, and "split bias," in which both positive and negative bias was applied at the same time to the nand p-side, respectively. The burn-in stations needed a separate VME crate to house the high voltage sources to bias the detectors. For the one-SASeq test stations, the HV module was connected to the same VME crate as the SASeq.

The HV VME crate for the burn-in stations contained four VME 4877PS [25] motherboards and a slave vertical interconnect board for high voltage crate 
control. Every motherboard carried eight HV pods. Thus the HV crate provided 16 positive and 16 negative independent voltages for silicon detector biasing and supported the current monitoring. The 4877PS Motherboard allowed the voltage to be set from 0 to $5000 \mathrm{~V}$, with a maximum current of $2 \mathrm{~mA}$ per channel. To ensure the safe operation of the burn-in stations, the over-voltage hardware protection of the HV supply was set to $120 \mathrm{~V}$.

During testing, the HDI tails were inserted into Hirose connectors on the connector adapter boards. These boards contained the standard 3M 50 pin connectors and the Hirose connectors and were used as connector adapters between the signal cables coming from the ICs and the HDI tails. The ICs were connected to the adapter board by a $3 \mathrm{~m}$ long, 50-conductor coaxial ribbon cable with an impedance of $75 \Omega$.

The burn-in stations were outfitted with a cooling system to operate the detectors at low temperature. Up to 16 detectors were placed on shelves inside a regular rack that had been thermally isolated. The chiller temperature was set to $3^{\circ} \mathrm{C}$, and the detectors ran at temperatures between $5^{\circ} \mathrm{C}$ and $15^{\circ} \mathrm{C}$, depending on the number of chips on the HDI. Each detector module was placed on a custom made, $178 \times 229 \mathrm{~mm}$ aluminum plate, designed to accept all types of HDIs or detectors. Every aluminum plate was equipped with a pipe for cooling water and special holes to provide nitrogen flow through the box that enclosed the device under test. Two aluminum plates together with the connector adapter boards and signal cables were placed on a plywood board. This board was equipped with sliders to simplify loading and unloading of the devices under test. Plywood was chosen because it was a cheap, low thermo-conductive material that helped reduce the condensation on surfaces inside the rack. Every board had its own water and nitrogen pipe. The control over the water and gas flow was provided by a control panel outside the rack. A software based interlock system monitored the temperature on each device and shut down the power in the event that the temperature exceeded $50^{\circ} \mathrm{C}$. The aim was to stay below the glass transition temperature of the epoxy to avoid damage to the detector assembly.

\subsection{Testing of Detector Modules}

The testing of detector modules was the first attempt to read out and bias a completed ladder or wedge detector after module assembly. The testing also included some remediation of issues and is thus also referred to as "debugging." The likelihood for damage to occur during assembly, mainly during wire bonding, was substantial. About $25 \%$ of the assembled detectors failed to download or to read out correctly. In addition, the leakage current was unacceptably high for about $95 \%$ of the double-sided assembled detectors. The 
functionality of the detectors had to be restored before any further tests were done.

First the modules were visually inspected to ensure that no mistakes had been made during wire bonding and no mechanical damage had occurred. The electrical resistances between the active beryllium pieces and the HDI ground were measured, and if a resistance was greater than $10 \Omega$, the grounding was improved either with a small wire or directly with a silver epoxy trace.

The functionality test of the readout was done without applying bias to the detector. Download and readout failures were mainly caused by damaged SVXIIe chips, misplaced or missing wire bonds, or damaged HDIs. About $10 \%$ of the SVXIIe chips had to be replaced.

Once a detector module had been successfully read out, it was biased. The biasing scheme depended on the type of detector under consideration. For the single-sided detectors, positive bias was applied to the n-side, with no segmentation. Because single-sided detectors do not have the bias applied on the coupling capacitor, the leakage current was low. No repairs of the single-sided detectors were needed, since they would remain operational even if a capacitor was broken during the wire bonding. Double-sided detectors were "split biased" by applying positive bias to the the n-side and negative bias to the p-side, corresponding to the axial strips for the ladders. Broken AC coupling capacitors caused by flaws in the lithography caused holes in the insulator, called pinholes. If one of the AC coupling capacitors failed for a channel, leakage current traveled directly through the readout electronics connected to the broken AC capacitor. This caused high leakage current for the detector, even at low bias voltages. Typically, the leakage current was as high as $100 \mu \mathrm{A}$ at a bias voltage of about $10 \mathrm{~V}$ applied on the n-side during testing. To remedy this, channels with broken AC coupling capacitors were identified and disconnected from the readout electronics by pulling the wire bond between the silicon sensor AC bonding pad and the SVXIIe preamplifier.

To identify the broken AC coupling capacitors at one side of the detector, bias voltage was applied on that side and the other side was kept grounded. Typical plots of pedestal, noise (defined as pedestal fluctuations for each channel), and differential noise (defined as fluctuation of the noise difference between two consecutive channels) are shown in Fig. 31 for a chip containing one broken AC capacitor. Broken AC coupling capacitors caused the input of the preamplifier to be dominated by the DC component, resulting in an almost constant output of the preamplifier. Consequently, the level of noise was low for broken channels and high for the neighbors as shown in Fig. 31, This phenomena is further discussed in Ref. [26]

The bonds for broken capacitors were pulled, and after that the positive bias 


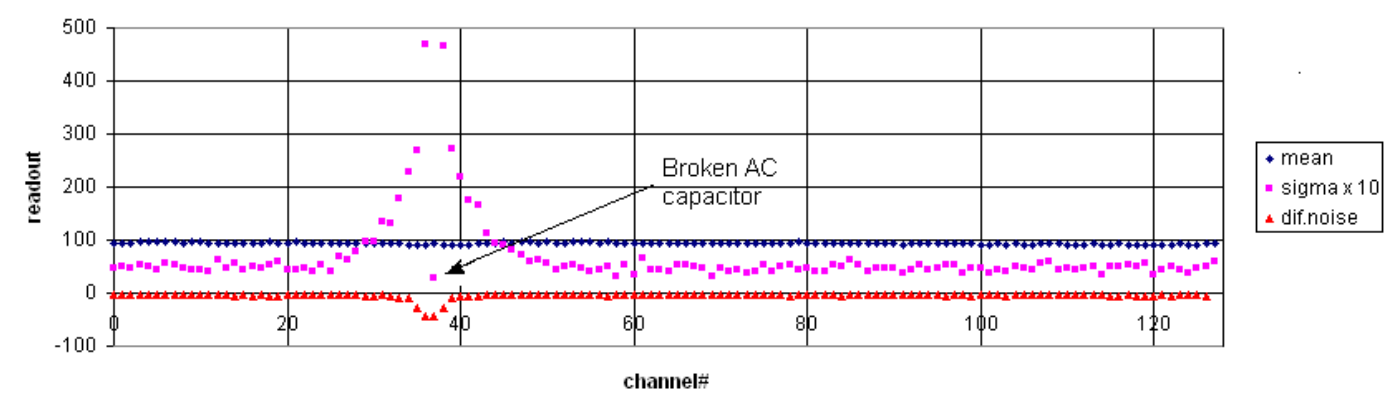

Fig. 31. Distributions of pedestal(diamonds), noise (squares), and differential noise (triangles) for a chip containing a broken AC capacitor. The noise (sigma $\times 10)$ is low for broken channels and high for neighboring channels. The differential noise is plotted multiplied by -1 for better visual clarity.

voltage applied to the n-side could be raised up to $V_{T S}+30 \mathrm{~V}$. $V_{T S}$ is defined as the depletion voltage measured on the test structure, and it was determined from the sensor probing discussed in Sec. 5. The leakage currents at the test voltage $V_{\text {test }}$, defined as $V_{T S}+20 \mathrm{~V}$, or $90 \mathrm{~V}$, whichever was lower, were measured to be in the range from $5 \mu \mathrm{A}$ to $25 \mu \mathrm{A}$.

A few detectors with high leakage current did not have any broken AC coupling capacitors. It was concluded that the leakage current was due to defects in the bulk silicon of the sensors, which could not be repaired.

The leakage current at depletion voltage was one of the considerations in the final electrical grade of the detector. Detectors with very high leakage current were not installed in the SMT.

One additional consideration for the detectors from Micron was that the negative bias voltage applied to the p-side was severely limited by micro-discharges [16]. For a module with no broken AC coupling capacitors, $V_{\max }^{-}$was defined as the highest voltage that could be applied on the p-side before the current started to grow exponentially. Above this value the leakage current, and also the noise, increased rapidly. In the absence of micro-discharges, $V_{\max }^{-}$was set to $\frac{1}{2} V_{\text {test }}$.

For the F-wedges, three additional problems were observed. The sensors had a tendency to develop pinholes during the first half hour of a burn-in test, resulting in high currents. In order to limit the time spent on burn-in, the wedges first were biased at the test station at $V_{\text {test }}$ and left at that voltage for 30 minutes. A second problem which developed for the Micron F-wedges was that there were regions in which pinholes seemingly propagated to several adjacent strips. When examining these wedges under a microscope, p-stop implantation faults were seen in the affected areas. However, this correlation was not complete, as some wedges affected did not exhibit visible faults. Probably 
this problem was linked to faults in the masking procedure of the silicon. The third problem that affected Micron F-wedge modules was related to the stability of the bias currents. The Eurysis F-wedge sensors generally exhibited fewer problems than the Micron sensors. While the Micron sensors in most cases had problems on the n-side, the Eurysis sensors exhibited a larger number of pinholes on the p-side. In addition pads that only should be used for bonding had been used to probe the sensors, thus the chips were bonded to pads with probe marks. For these sensors, the I-V characteristics showed a clear junction breakdown when applying negative bias.

One problem of concern was the general appearance of micro-discharge at around $70 \mathrm{~V}$ in positive bias, which indicated that the high voltage would be limited to less than the design value for the wedges. This could become an issue after type inversion if the depletion voltage needs to be increased past the micro-discharge voltage.

For double-sided detectors, $0.8 \%$ of the channels were disconnected from the readout electronics during the debugging process. The bare silicon sensors were tested before assembly, and only working channels were bonded. It was thus concluded that the capacitors broke during wire bonding.

The debugging was the most time consuming step of the production testing effort, and therefore its bottleneck.

\subsection{Burn-in Tests}

The burn-in test was a long-term functionality test performed during the production testing. First, the burn-in test was done on the bare HDI after its initial functionality test. The second burn-in test was carried out after ladder or wedge assembly.

The goal of the burn-in test was to run each HDI or detector module for 72 hours, monitoring its performance and measuring pedestals, total noise, random noise, gain, and occupancy in sparsification mode. Additional parameters were monitored, including temperature, SVXIIe chip current for the three SVXIIe chip voltages, and for ladders and wedges, also detector bias voltage and dark current. Typical problems revealed by the burn-in test were SVXIIe chip failures, broken and shorted bonds, grounding problems, noisy strips, and coupling capacitor failures. The burn-in setups were able to accommodate 32 devices. Two burn-in cycles were run per week, which allowed keeping up with the peak production rate of $\approx 20$ detector modules per week. Several different steps were incorporated into the burn-in tests, and they are briefly described below. 


\subsubsection{Temperature Sensor Test}

The goal of the temperature sensor test was to check the operation of the temperature sensor mounted on each HDI. The temperature sensor is a thermal resistor, and its voltage is digitized and converted into temperature. The temperature was measured five times with an interval of 20 seconds between measurements. The test was performed with all readout electronics off and with the sensor at room temperature. The most common failure of the temperature test was due to a bad contact of the thermo-resistor to the HDI. These HDIs were easily repaired.

\subsubsection{Data Integrity Check}

The goal of the data integrity test was to check the SVXIIe chip downloading. The SVXIIe chips were downloaded about 100 times. For each download cycle, the program compared the initial download file with the one read back from the chip and counted the errors. The parameters that were compared included chip ID, channel number and sequence, and pedestal values. Only those HDIs that had no download errors were accepted for future use.

\subsubsection{Long Burn-in Test}

During the long burn-in tests, the devices were left powered and biased for 72 hours. Bare HDIs were run on an aluminum plate that acted as a heat sink, and effectively operated at approximately $30^{\circ} \mathrm{C}$. Higher temperatures could prevent the SVXIIe chips from downloading reliably. Modules were run on a cooled plate, and operated at about 15 to $20^{\circ} \mathrm{C}$, depending on the number of chips. During the burn-in process, each device was sequentially tested in so-called runs. Overall, ten runs were taken. Each individual test took approximately three minutes per chip. i.e. 10 minutes for each 3-chip ladder, 20 minutes for each 6-chip ladder, etc. Once the last device had been tested for the first time, the system would wait for a user determined delay. During that time the units were left powered and biased, and monitoring information was collected. Several tests were performed during each run. They are summarized below.

The run started by taking one hundred pedestal events in data modes to obtain the average pedestal and random noise of the device under test. The pedestal mean and its width were also recorded for each readout channel separately and average values determined. For every event, the common line shift (average pedestal over 128 SVXIIe chip channels) was also calculated. This was used to record common mode subtracted pedestals for every channel, from which the random noise was determined. This sequence was repeated

with the chip in cal_inject mode, to obtain pedestal values to be used during 
the chip calibration.

The next step was the chip calibration. Each channel of the SVXIIe chip was connected to a built-in test input capacitor. The test capacitor was used for gain studies, during which a known charge was injected into each channel. Every eighth channel was pulsed simultaneously in order to not to get cross talk from adjacent channels and at the same time have a reasonable run time for the test. Four calibration pulses with different charges were applied one after another to the chip. One hundred events were recorded for each value of the calibration voltage. The same analysis cycle was repeated for eight channel patterns so that the charge was injected in every channel of the SVXIIe chip.

The chips were also tested in sparsification mode. In this mode, only the channels with a response that exceeds the preset threshold and their immediate neighbors were read out and the frequency of false readouts was studied. The chip performance in sparsification mode was evaluated by counting the frequency of false readouts for every channel. The readout was called false if the channel appeared in the data more often than expected (noisy channels) or less often than expected (dead or low gain channels). For double-sided devices calibration and sparsification readout testing were performed separately for the p- and n-side.

Monitoring information was collected once per minute for the duration of the burn-in test. Bias voltage and current were measured for ladders and wedges directly through the high voltage power supply.

The burn-in test proved to be an efficient and reliable tool to test the long term reliability of the detector modules before installation into the SMT. Bare HDIs were selected for detector construction based on the results of the burnin test. Together with the laser test, the burn-in test was used to characterize the detector modules and select those devices to be installed in the tracker. Over a thousand HDIs and detector modules were tested during the SMT production testing project. Taking into account the average load of $75 \%$, this corresponds to approximately 6,000 hours of stable running by each of the two burn-in stations.

\subsection{Laser Test}

The laser test was performed on every detector module that passed the burnin test. The modules were characterized by measuring the depletion voltage and by determining the numbers of dead and noisy channels. This information had already been obtained by other tests: the burn-in was able to identify dead and noisy channels, and the probe tests on the sensors determined their depletion voltage. However, early tests indicated discrepancies between the 
depletion voltage measured during sensor probing and the one obtained on the assembled module. This led to the implementation of the laser test, and although it provided redundant information, it was considered a useful tool in the characterization of modules. This was particularly true in the characterization of irradiated detectors, to study noisy strips, and to investigate irregular charge collection observed on some ladders. The test stand was set up to run completely automatically and was very fast.

A simplified diagram of the laser test stand is shown in Fig. 32. It was based on the one-SASeq test stand, with the addition of an infrared laser and a movable table. The solid state laser had a wavelength of $1064 \mathrm{~nm}$. Given the attenuation length of the silicon of $206 \mu \mathrm{m}$, the laser penetrated the entire depth of the $300 \mu \mathrm{m}$ thick detector and not just a surface layer. The laser was connected to an optical fiber. At the end of the fiber, a focusing lens collimated the laser spot. Completed modules were placed on an $x-y$ movable table and shuttled underneath the laser head. Two laser test stands were used during production.

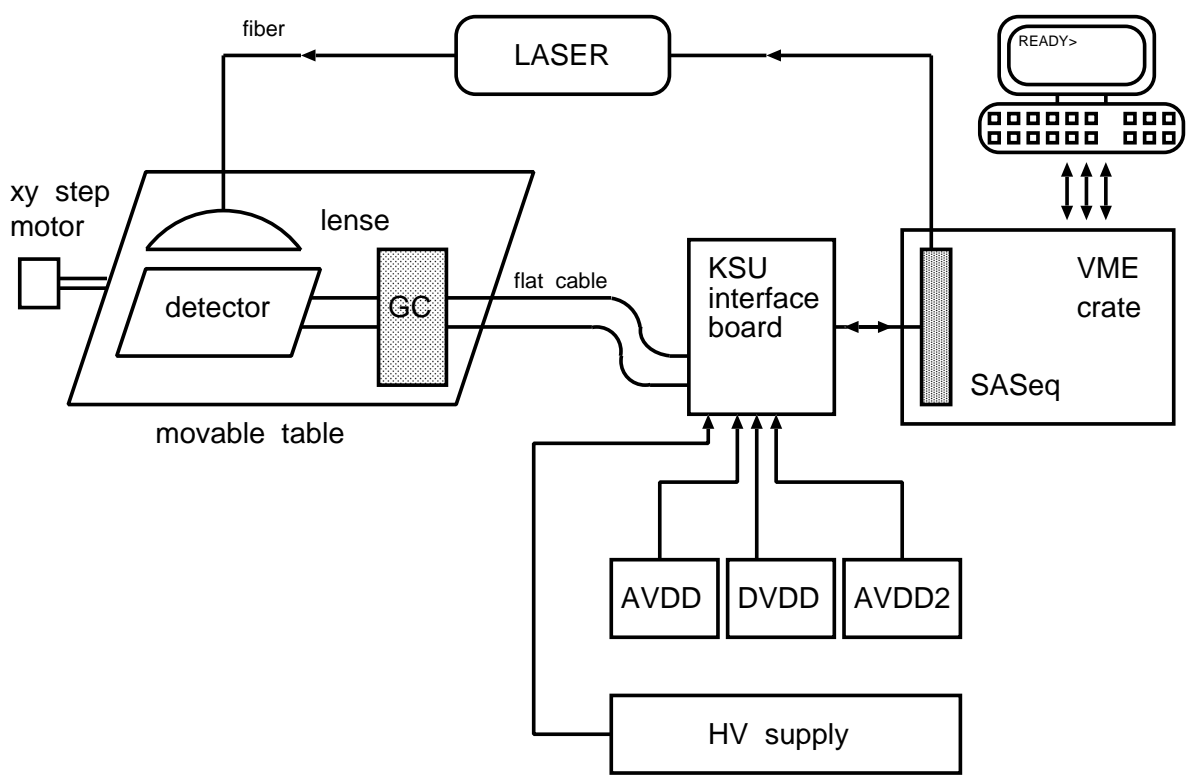

Fig. 32. Simplified scheme of the laser test stand setup.

The depletion voltage was measured by monitoring the charge collected on the readout strips that detected the laser, as a function of the bias voltage. The procedure for the single-sided detectors was the following: first, the range of the voltage variation was set from $0 \mathrm{~V}$ to $V_{\text {test }}$. Then, the bias voltage was increased automatically in steps of $5 \mathrm{~V}$, and after each step the amplitude of the laser signal in ADC counts was measured. The leakage current of the detector at each voltage setting was also recorded. We found an almost linear increase of the amplitude $A$ of the signal with the bias voltage $V$ up to a value $V_{\min }$, after which the $A(V)$ curve reaches a plateau. As a first approximation, the $A(V)$ curve can be described by a slope and a plateau that fit the data linearly. The 
depletion voltage was defined as the voltage corresponding to the point where the two linear fits intersect. The results using this method for determining the depletion voltage $V_{\text {depl }}$ was in most cases in good agreement with the results obtained with the $\mathrm{C}-\mathrm{V}$ method of the depletion voltage measurements used on silicon sensors. The minimal operating voltage was defined as the voltage at the beginning of the plateau. The operating voltage $V_{\text {oper }}$ of the detector was obtained by adding $5 \mathrm{~V}$ to the minimal operating voltage. The procedure was very similar for double-sided detectors.

The 3-chip ladders, the 9-chip ladders, and the H-wedges which consist of two silicon sensors had their strips wire-bonded by pairs to each other. For these devices, the depletion voltage was measured separately for the two sensors. Because sensors were paired into detectors based on their depletion voltage as determined during sensor probing, these two measurements were in general very close. The higher of the two operating voltages was taken as the detector operating voltage.

The laser scan was also used to identify dead and noisy channels. This was performed with the detectors biased at $V_{\text {oper }}$, as determined from the laser test. Every channel was read out ten times resulting in a set of raw amplitudes. The average amplitude and the standard deviation were calculated over the ten measurements.

Each detector module was tested with the laser at least once during the production testing effort. The information obtained was used as input for the grading of the modules.

\subsection{Diagnosis and Repair of Defective Modules}

HDI and detector modules with download or readout problems had to be diagnosed and repaired. A total of 339 problematic HDIs and detectors were found, and most of them were also repaired. Although some of the repairs were performed by the physicists working on diagnosing the problems, most of the repairs required work done by a specially trained technician. Malfunction of HDIs was usually due to problems during stuffing at the commercial vendors, or bad packaging before shipping. Detectors were also damaged during the production and testing process, or during installation on the rings or on the barrels.

The stations used for the diagnosis and repair effort were based on the oneSASeq test stations with the addition of a microscope, a probe, an oscilloscope, and a logic analyzer. This additional equipment made it possible to probe the signals on the SVXIIe chips directly. One very important feature of the diagnosis stations was that on the IC, the top neighbor and bottom neighbor 
lines were disconnected, and the signal was sent to the SVXIIe chips via the probe. This made it possible to download the chips one by one, which turned out to be a very effective tool to find bad chips on a device that was showing download errors.

The procedure to diagnose modules with problems was the following:

(1) Visual inspection to look for broken or shorted bonds. On HDIs that failed to work properly at the initial functionality test, the visual inspection was extended to check that all capacitors and resistors were mounted properly.

(2) Check the HDI tail for shorts between the three voltage power lines and ground. Shorts tend to develop at the trimmed end of the tail of the HDI and could be fixed by sanding the tail with sandpaper.

(3) Shorts would also develop due to solder on the tail or under a surface mounted component making contact with other pads. Several H-wedge HDIs developed shorts between one trace and ground due to sparks from a capacitor between the bias voltage line and ground to this trace.

(4) Broken traces on the HDI could in most cases be repaired by adding an extra wire. This kind of problem was very common on the H-wedges, for which traces often broke close to the Hirose connector that was mounted on the HDI tail.

(5) Shorts on the SVXIIe chips themselves were identified by first isolating the faulty chip by pulling the relevant bonds, and then measuring the resistance between the shorted channel and ground. If a short was found on a chip, the chip was replaced.

(6) If no short was found between the power lines and ground, the next step was to try to download the chips. Usually at least one of the chips would give download errors. To identify the problem, the lines were checked with the logic analyzer.

(7) To further investigate which chip had download errors, chips were downloaded individually. Chips were isolated by pulling the top neighbor and the bottom neighbor bonds. When the chip with the download errors was identified, it was checked once more for bad bonds and if none were found, the chip was replaced.

(8) If a chip was downloading correctly but had a readout problem such as no gain, it was replaced.

(9) Occasionally, detectors would stop working after encapsulation of the wire bonds. In those circumstances, the encapsulant was removed, which caused the wire-bonds to come off too, and the detector re-bonded.

(10) On some of the detectors, neighboring chips were damaged during replacing of a bad chip. This was especially common for the F-wedges.

Of the 339 devices (181 bare HDIs and 158 detectors) that were diagnosed by the repair group, 264 were fully recovered. From these, 74 had only minor problems, such as bad grounding or missing or damaged bonds. Thirty-eight 
detectors could not be repaired, and for 37, the repair was not attempted, because enough spares of the same type were available. A total of 288 SVXIIe chips was replaced: 145 because of readout problems, 83 did not download, and 60 had a short. A total of 14 components was found missing on the HDIs. Problems with the tail or the traces on the HDIs occurred for 51 devices.

The types of devices with the most problems were F-wedges and 9-chip ladders: 113 and 114, respectively. Seventy-eight H-wedges, 32 6-chip ladders, and seven 3-chip ladders were sent to the repair group. F-wedges often took a long time to repair since they were frequently damaged during repair. H-wedges were difficult to diagnose since they often had many broken traces close to the connector on the tail.

The effort of the repair group was essential to completing of the SMT because there were not enough spares to replace broken detector modules or nonfunctioning HDIs.

\subsection{Electrical Grading and Characteristics of Detector Modules}

The results collected during detector burn-in and laser test were used to assign an electrical grade to each detector module. We defined two types of "exceptional" channels:

- A channel was called DEAD if its response to the laser in the laser test was less than 40 counts (the average response was 100 counts). For detectors with two sensors, the one with the larger count of dead channels was considered in the electrical grading.

- A channel was called NOISY if its random noise in data mode during burn-in was larger than 6 counts (average noise was 1.5 counts)

A detector was assigned an "A" grade if the sum of the number of dead and noisy channels was less than $2.6 \%$ of the total number of channels. This corresponds to 10 channels for a 3-chip ladder, 20 channels for a 6-chip ladder, etc. No distinction between n-side and p-side bad channels was made in the grading. A detector was assigned a "B" grade if the sum of the number of dead and noisy channels was more than $2.6 \%$ but less than $5.2 \%$. Detectors with more than $5.2 \%$ bad channels were graded as "C." In addition, we required that the leakage current be less than $10 \mu \mathrm{A}$ at operating voltage and that the burn-in results were stable over time. Mostly "A" grade detectors were installed in the SMT, no "C" grade detectors were installed.

The performance of detector modules installed in barrels and F-disks is summarized in Table 10 and Table 11, respectively. 
Table 10

Fractions of dead and noisy channels in barrel detectors given in percent.

\begin{tabular}{|c|c|c|c|c|c|c|c|c|}
\hline \multirow{2}{*}{ Barrel } & \multicolumn{2}{|c|}{ Layer 1 } & \multicolumn{2}{c|}{ Layer 2 } & \multicolumn{2}{c|}{ Layer 3 } & \multicolumn{2}{c|}{ Layer 4 } \\
\cline { 2 - 9 } & Dead & Noisy & Dead & Noisy & Dead & Noisy & Dead & Noisy \\
\hline 1 & 0.85 & 0.4 & 1.99 & 0.8 & 0.93 & 0.4 & 1.77 & 0.6 \\
2 & 0.95 & 0.56 & 2.13 & 0.43 & 1.34 & 0.22 & 2.01 & 0.44 \\
3 & 0.70 & 0.30 & 1.4 & 0.4 & 0.77 & 0.12 & 1.27 & 0.3 \\
4 & 0.56 & 0.18 & 2.1 & 0.5 & 0.84 & 0.06 & 1.50 & 0.2 \\
5 & 0.77 & 0.51 & 1.65 & 0.27 & 1.22 & 0.32 & 1.54 & 0.2 \\
6 & 1.1 & 0.07 & 2.7 & 0.4 & 1.48 & 0.12 & 1.85 & 0.16 \\
\hline
\end{tabular}

Table 11

Fractions of dead and noisy channels in F-disks given in percent.

\begin{tabular}{|c|c|c|}
\hline Disk & Dead & Noisy \\
\hline 1 & 0.92 & 0.23 \\
2 & 1.0 & 0.21 \\
3 & 1.75 & 0.05 \\
4 & 1.0 & 0.32 \\
5 & 1.65 & 0.05 \\
6 & 1.02 & 0.25 \\
7 & 1.25 & 0.33 \\
8 & 1.4 & 0.13 \\
9 & 1.22 & 0.45 \\
10 & 1.32 & 0.15 \\
11 & 1.15 & 0.45 \\
12 & 1.25 & 0.35 \\
\hline
\end{tabular}

It can be seen that the characteristics of the devices are very similar throughout the different barrels and disks, resulting in a uniform acceptance of hits produced by charged particles. 


\section{Assembly of the Central Detector}

The central detector assembly was built in two cylinders. Each cylinder contains four sub-assemblies, three barrel-disk modules, and one end-disk module. This section begins with descriptions of the assembly of the barrels and the F-disks. Following these is a description of the mating procedure used to assemble these elements into the two module types, barrel-disk and end-disk. The final subsection covers the installation and alignment of these modules in the support cylinders.

\subsection{The Barrel Assembly}

The primary mounting of the ladders was to the cooled (active) bulkhead, with a secondary mounting point at a thin membrane (passive bulkhead). The cooled bulkhead is the primary mechanical object, supporting the F-disks mated to the barrel and providing the connections to the support cylinder. The ladders themselves form the mechanical connection between the two bulkheads so that the passive bulkhead serves as a precise spacer at the cantilevered ends of the ladders.

The barrel detectors are used in the Level 2 track trigger [6], which utilizes mainly the $r-\phi$ information to look for tracks with large impact parameter. The polar angles of the tracks are not available to the trigger. Precise placement of the sensors is therefore essential to maintain the trigger hit resolution. The constraints on assembly accuracy are discussed in the next section.

The beryllium plates in the ladders were used to locate the ladders on precisely machined beryllium bulkheads. The original intent was to have no adjustment in this mounting, but machining precision and ladder assembly precision were not sufficient, so that some alignment was done during ladder installation, as described below.

The survey data recorded as part of the quality assurance during assembly, described in detail below, provided a very good starting point for off-line alignment with tracks.

\subsubsection{Requirements on Assembly and Survey Accuracy}

Deviations from the ladder nominal position can be characterized by three shifts and three rotations. If the deviations are small, rotations effectively commute so all six parameters were considered independently. 


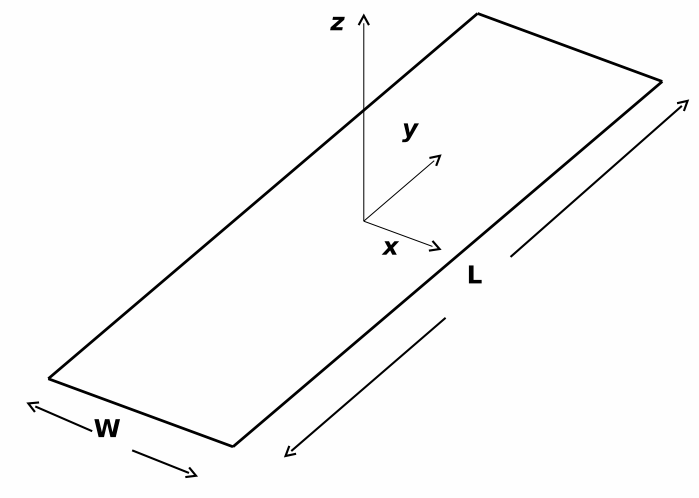

Fig. 33. Ladder and its local coordinate system. Axial strips run along the ladder (y-axis).

Shifts $\Delta_{x}$ and $\Delta_{y}$, with the local coordinate system given in Fig. 33, result in mis-measurements that are uniform across the ladder and equal to the shifts. A shift $\Delta z$ and all rotations result in mis-measurements which are functions of the position within the ladder.

The rotations in the $x y$ and $y z$ planes introduce mis-measurements which depend on $y$ and therefore can not be corrected at the trigger level. These two rotations dictated the assembly precision required. The dominant impact parameter uncertainty at the trigger level is the beam size (about $30 \mu \mathrm{m}$ ), and alignment errors should not compromise it. Detailed simulations were performed to see how alignment errors in individual layers propagate to the error on the impact parameter. The misalignment of the geometry was simulated one layer at a time, with the limit of the impact parameter error to be less than half the beam spot size. Each layer was allowed to contribute equally to the impact parameter error. This led to allowed limits for the misalignment of 10, 15, 20 and $15 \mu \mathrm{m}$ for layers 1 through 4 . Since the simulation assumed $100 \%$ hit efficiency, we conservatively took $10 \mu \mathrm{m}$ to be the limit on alignment errors in each layer which translates into

$$
\begin{gathered}
\Delta_{x y}=25 \mu \mathrm{m} \\
\Delta_{y z}=160 \mu \mathrm{m} \text { for layers } 1 \text { and } 2 \\
\Delta_{y z}=320 \mu \mathrm{m} \text { for layers } 3 \text { and } 4,
\end{gathered}
$$

where $\Delta_{x y} / \Delta_{y z}$ is the rotation in the $x y / y z$ plane corresponding to the movement of the edge of the ladder. In the calculations done in order to reach these numbers, perfectly planar ladders were assumed. Two deviations from planarity were further considered: "tent"-like shape [Fig. [34(a)] and a twist 
[Fig. [34(b)].

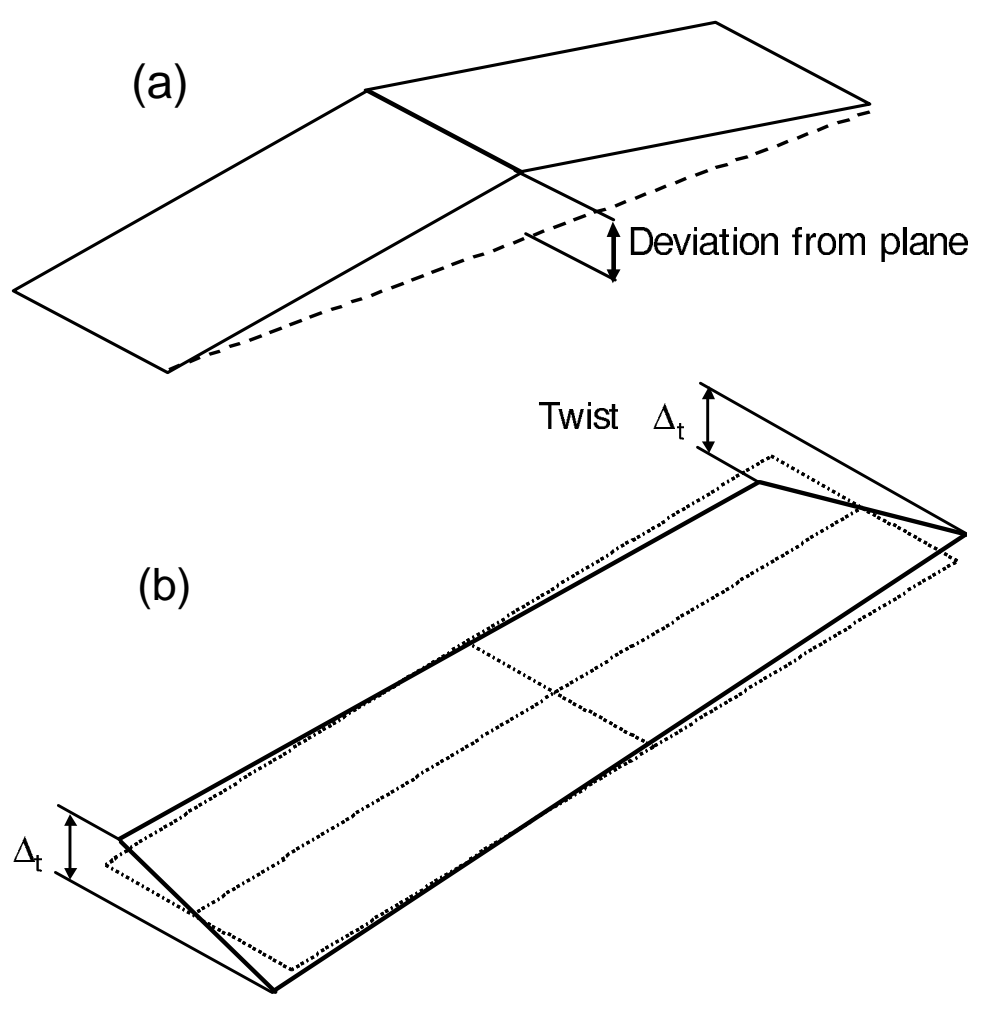

Fig. 34. Ladder deviations from plan: (a) "Tent"-like deviations and (b) twist.

The former contributes to the trigger resolution in the same way as $\Delta_{y z}$. The effect of twisting is more than a factor of three smaller than that from $\Delta_{x z}$. Offline, however, these shapes are considerably more difficult to incorporate into software corrections and in situ alignment algorithms using tracks. This means that these effects may also impact not only the trigger resolution, but also the final offline tracking resolution. Therefore these contributions were kept to a minimum during detector assembly.

\subsection{Measurements of Ladders}

The main purpose of the ladder survey was to provide a link between the optical targets printed on the silicon sensors and the mechanical elements of the ladder structure. These elements could be surveyed with a touch probe after the ladders were installed in the barrels and the optical targets were no longer accessible. An important byproduct of these measurements was quality control of ladder planarity. These measurements were all done on an optical CMM with automated pattern recognition and auto-focus capabilities. The machine accuracy was less than $2 \mu \mathrm{m}$ in the $x y$ plane and less than $5 \mu \mathrm{m}$ in $z$ when measuring distinctive features such as the metalization on the sensors. 
Figure 35 shows the measurement points taken on 9-chip ladders. Measurements of 3- and 6-chip ladders were similar.First, six silicon fiducial markers, noted by + and a number in the figure, were measured. They were fit to a plane, which was defined to be the $x y$ plane of the measurement. The origin of the coordinate system coincides with fiducial 1 with the $y$ axis passing through fiducial 2. Ladder flatness was defined as the minimum separation between two planes parallel to the $x y$ plane which would contain all six measured points. It was defined to be positive if the ends of the ladder were below the $x y$ plane (as in Fig. 34a) and negative otherwise. Figure 36 shows the flatness distribution for ladders installed in the inner two layers of the detector.

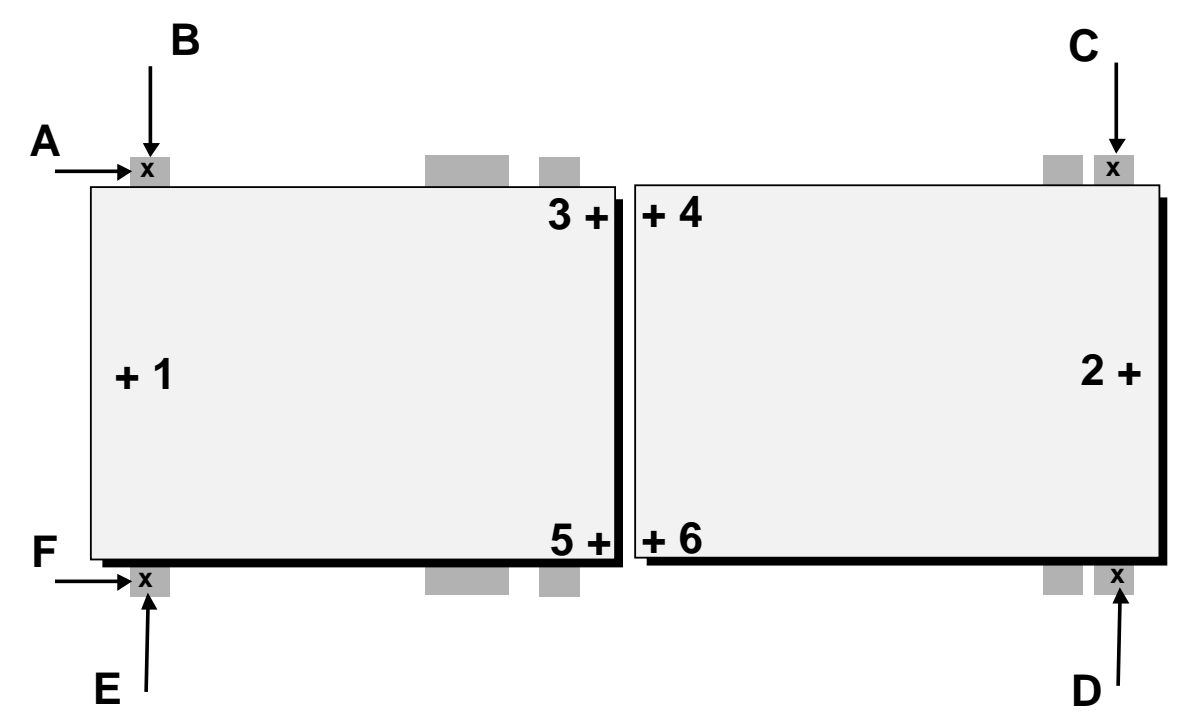

Fig. 35. Ladder measurements. Lighter gray rectangles are silicon crystals, darker gray are beryllium support. Silicon fiducials are noted by + with a number; measurement on a beryllium surface by $\mathrm{x}$; measurement of beryllium edges with arrows and letters.

After the coordinate system was established, the beryllium surfaces (noted by $\mathrm{x}$ ) and edges (noted by arrows with letters) were measured (see Fig. 35). The edges of the beryllium supports were not perfectly machined and generally were not square (see Fig. 37). It was therefore necessary to make a scan across the edge. With the optical probe, three measurements were taken at the three different focus points indicated in Fig. 37. The average of the three was taken as the final measurement. For touch probe measurements, a scan with a $50 \mu \mathrm{m}$ step was made, with a probe diameter of $1 \mathrm{~mm}$, and the largest measurement was used.

The edges of the beryllium can have microscopic bumps with a typical size of 20-30 $\mu \mathrm{m}$. Figure 38 schematically shows the effect such bumps can have on the measurements. Defects like this were not frequent, but as a precaution three scans were performed at each location, separated by $100 \mu \mathrm{m}$. 

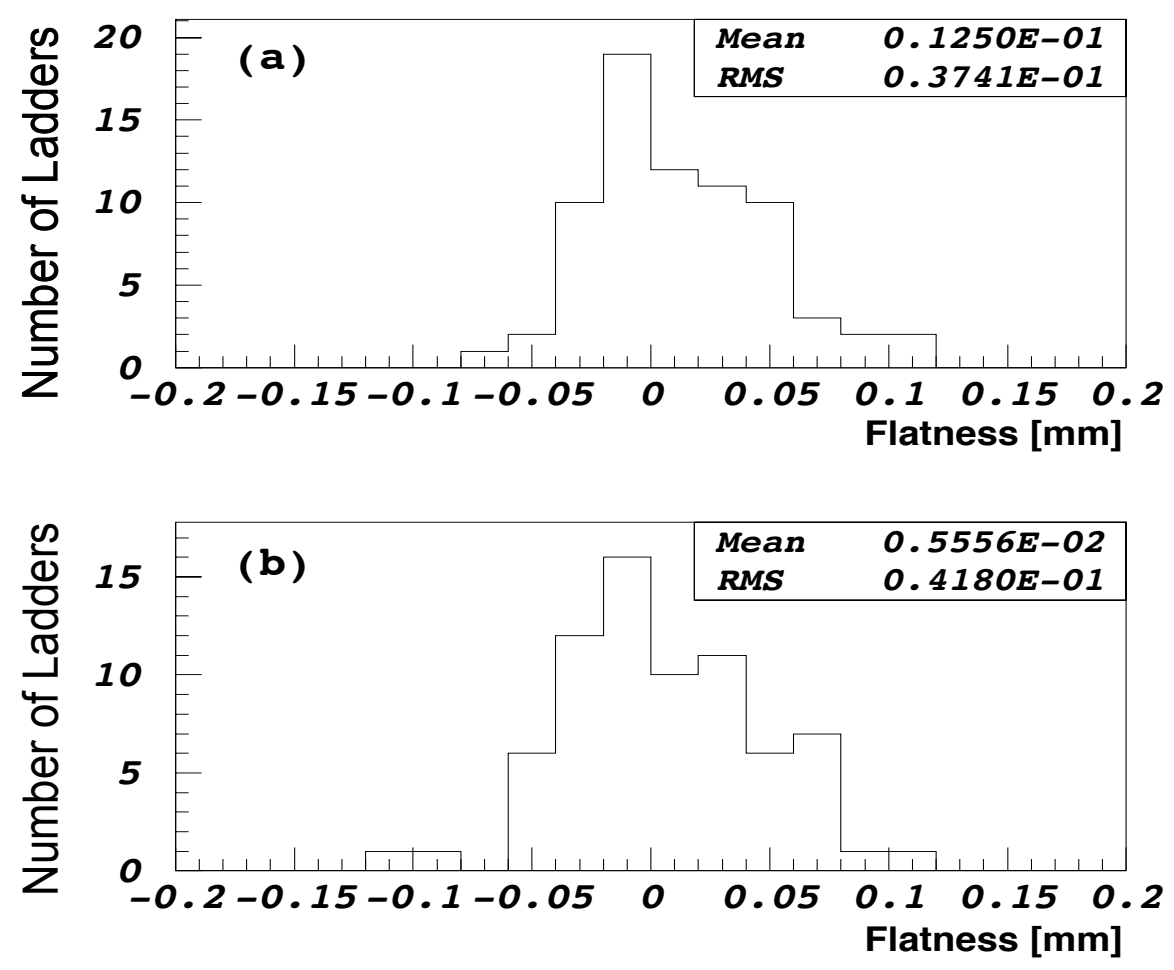

Fig. 36. Ladder flatness for ladders in (a) layer 1 and (b) layer 2.

Since the systematic errors associated with the optical and touch probe methods were different, the comparison of these measurements provided a very powerful consistency check. Figure 39 shows the difference between the ladder widths determined by the touch probe and optical methods. The absence of a high tail in the distribution confirms that edge imperfections are not frequent. The distribution is centered at $-17 \mu \mathrm{m}$, i.e. on average the touch probe gave smaller widths. This effect was studied in great detail and was attributed to the edge finding algorithm, as well as the bias introduced by choosing the average of the optical measurements at three focal depths versus the largest of the touch probe measurements taken through the thickness. However, this systematic bias does not contribute to the accuracy of the ladder position which was determined by pairs of measurements taken on opposite surfaces. Thus the ladder position accuracy was completely determined by the intrinsic accuracy of the CMM machine, about $6 \mu \mathrm{m}$, which was sufficient for quality control.

\subsection{Assembly and Survey Procedures}

The assembly process started with aligning active and passive bulkheads. Both were mounted on a rotating barrel support fixture. The cooled bulkhead was fixed in place and served as the primary reference system for measurements. 


\section{Optical Touch Probe}

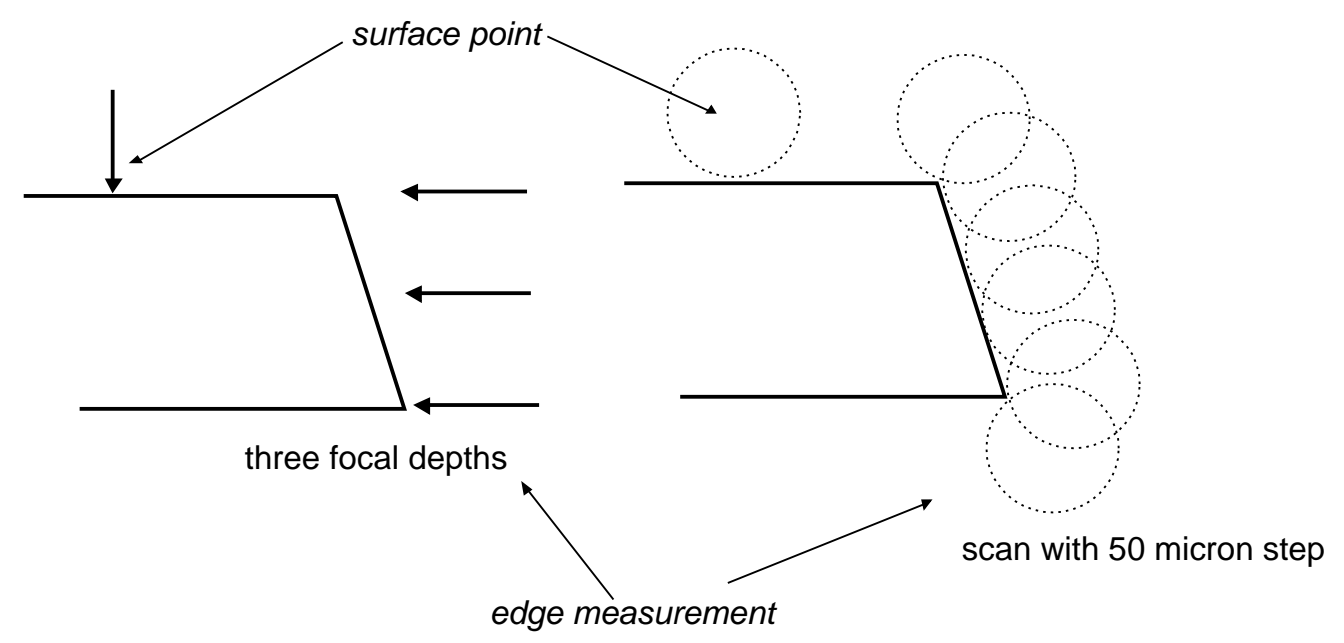

Fig. 37. Schematics of optical and touch probe measurements.

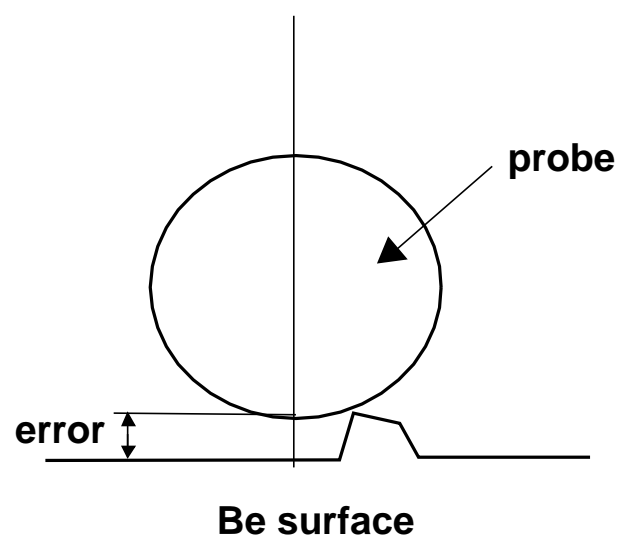

Fig. 38. Effect of edge defects on the match between optical and touch probe measurements. The optical measurement may not be affected by the bump, while the touch probe was.

The face of this bulkhead and precision holes at 3 and 9 o'clock were used to establish the reference coordinate system. Precision spacers were used to locate the passive bulkhead parallel to the cooled bulkhead and to match machined alignment holes at 3 and 9 o'clock. The passive bulkhead position was adjusted until it was within $10 \mu \mathrm{m}$ of nominal and clamped to the fixture. 


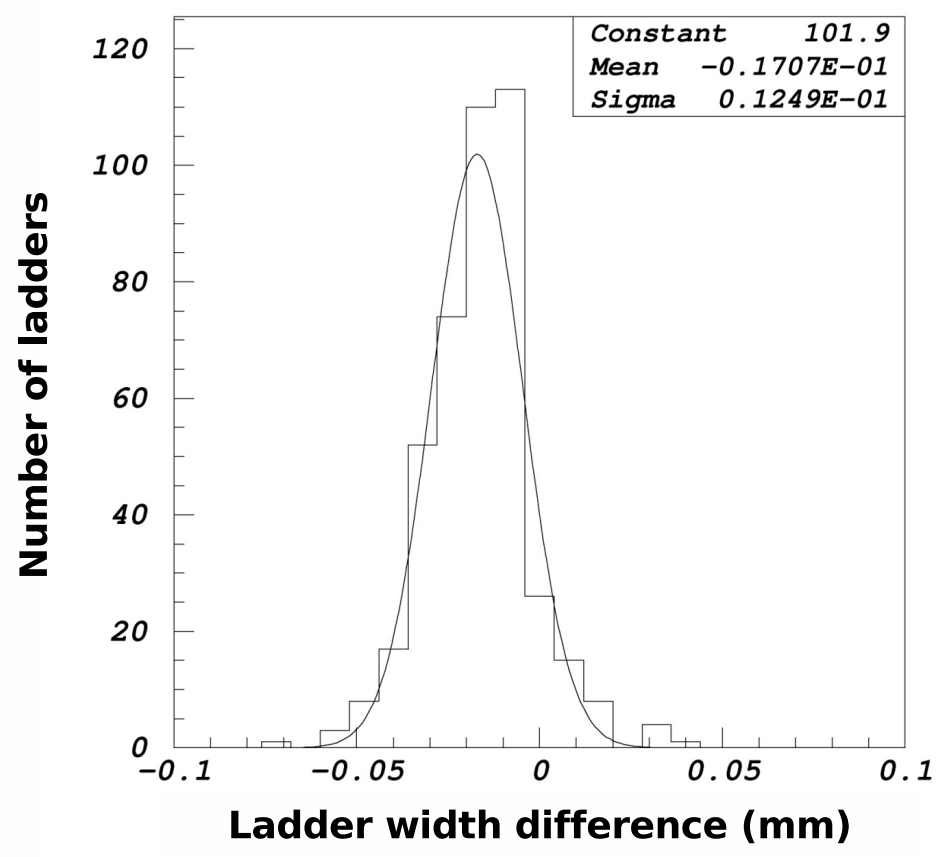

Fig. 39. Difference in ladder width as determined by touch probe and optical methods. Negative values mean that touch probe consistently gave smaller widths.

Next, the ladders were installed using an adjustable mounting fixture. The assembly sequence was determined by convenience of HDI pigtail routing. It was found that it was easiest to assemble the barrels from the inside out. All barrels except the first one were assembled this way. The surface where the ladders mate to the cooled bulkheads had thermal grease applied to enhance the heat transfer through these joints, with the exception of the layer 4 ladders which were glued in place using silver epoxy. These ladders then provided the rigid coupling between the cooled and passive bulkheads once the barrel was removed from the assembly fixture.

After installation of each ladder, an electrical test was performed to ensure that it was not damaged. The ladder was powered, nominal bias voltage was applied, and pedestal values were recorded. Bias current and low voltage currents were checked. The most common damage was to wire bonds either from contact with HDI tails from other ladders or during ladder position adjustment. In layer 4, the silver epoxy, which should hold the ladders in place, migrated in some cases and produced shorts along the exposed edges of the sensors. Damaged ladders were removed for repair and the assembly continued with other ladders.

After electrical testing, the ladder was surveyed with the touch probe. The touch probe measurements were then fit to the optical measurements taken previously. If the measured $\Delta_{x y}$ was more than $25 \mu \mathrm{m}$, the ladder was adjusted and resurveyed. Since it was not possible to control the adjustment, sometimes 


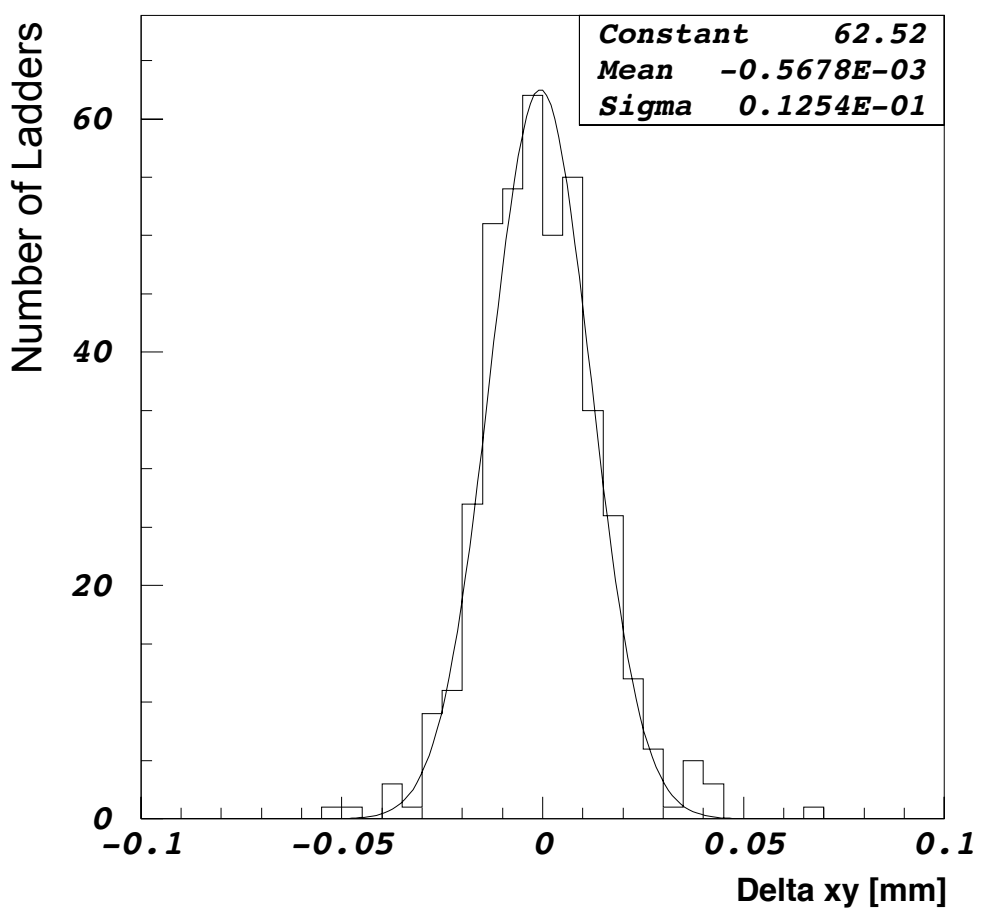

Fig. 40. Barrel survey summary: $\Delta_{x y}$. The width of the distribution is well below the required $25 \mu \mathrm{m}$.

several iterations had to be performed. In rare cases, it was impossible to reach the desired $\Delta_{x y}$ due to ladder or bulkhead imperfections.

When the whole barrel was assembled, dabs of silver epoxy were added to the ladders in layers one, two, and three to ensure electrical contact between the beryllium bulkheads and the ground of the HDI electronics. This also improved the mechanical stability of the barrels. All ladders were again checked electrically, and the final survey of the ladders was performed. Prior to the final survey, the HDI tails were permanently strain-relieved to a carbon fiber hoop mounted around the outer perimeter of the cooled beryllium bulkhead. The full barrel survey was a 2-3 day process for each of the six barrels. The distributions of $\Delta_{x y}$ and $\Delta_{y z}$ for all ladders in all barrels are shown in Fig. 40 and Fig. 41, respectively.

After all modules were surveyed, we performed the best fit to find a global coordinate system for the barrel, i.e. the system where the ladder rotations were minimal. This was done for all six barrels, and it was found that the best system was very close to the assembly system. After assembly, testing, and final survey of a barrel was complete, the barrel was removed from the assembly fixture and stored in a clean, dry air environment until it was mated with its F-disk. 

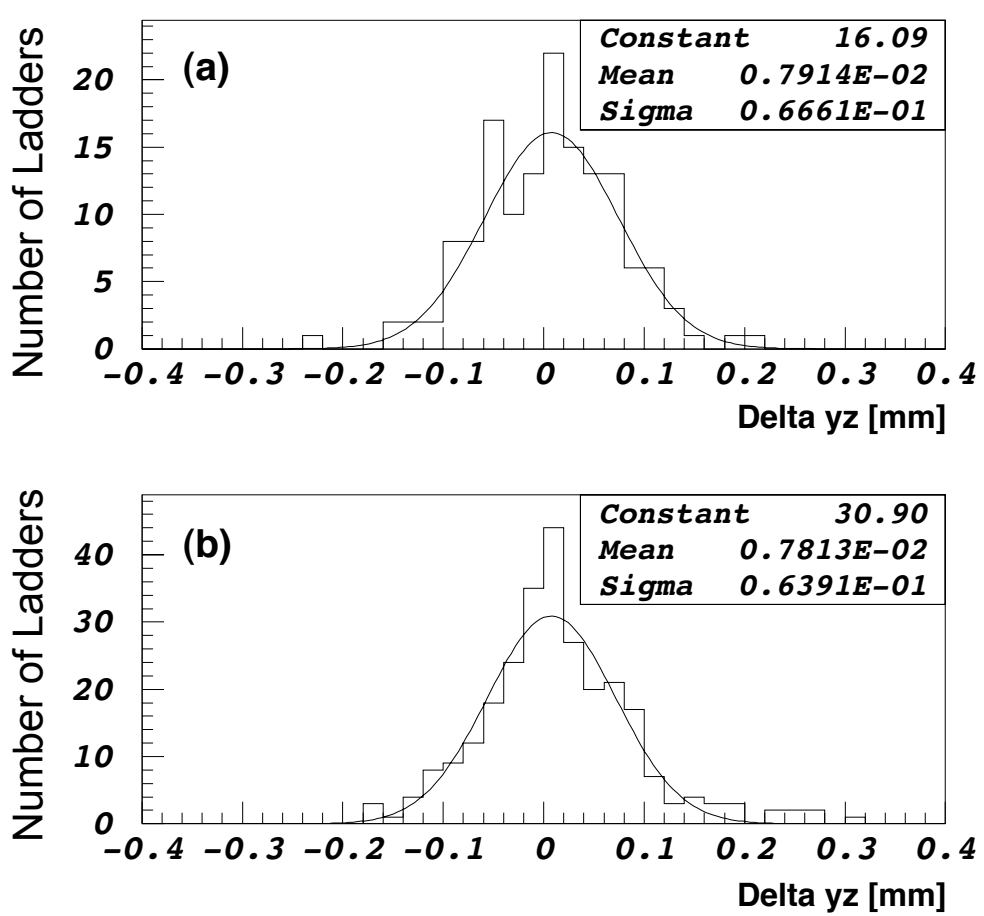

Fig. 41. Barrel survey summary: $\Delta_{y z}$. The width of the distribution is well below the required $160 \mu \mathrm{m}$ for layers 1 and 2 (a) and $320 \mu \mathrm{m}$ for layers 3 and 4 (b).

\subsection{The F-disk Assembly}

This subsection describes the assembly, survey, and quality control testing of the F-disks. Figure 42 shows a photograph of an F-disk assembly.

The F-disks were mounted to the barrels via carbon fiber posts with precision machined aluminum inserts which engaged holes in the barrels and attached with aluminum nuts on titanium studs. In the case of the end-disk modules, posts from two disks were mated with ruby balls and glued together.

\subsubsection{Beryllium Support Rings}

The beryllium support rings provide the infrastructure for the F-disks, including the mechanical mounts as well as the cooling. The rings were made from two pieces of beryllium laminated together. The beryllium ring was assembled with mounting and aligning hardware as well as plumbing connections.

The beryllium was machined by Phoenix Precision [27]. The most important consideration for this process was the flatness of the finished piece. Figure 43 shows the distribution of flatness for each of the two beryllium halves of the assembly. They range in flatness from $25-50 \mu \mathrm{m}$. The beryllium pieces contain 


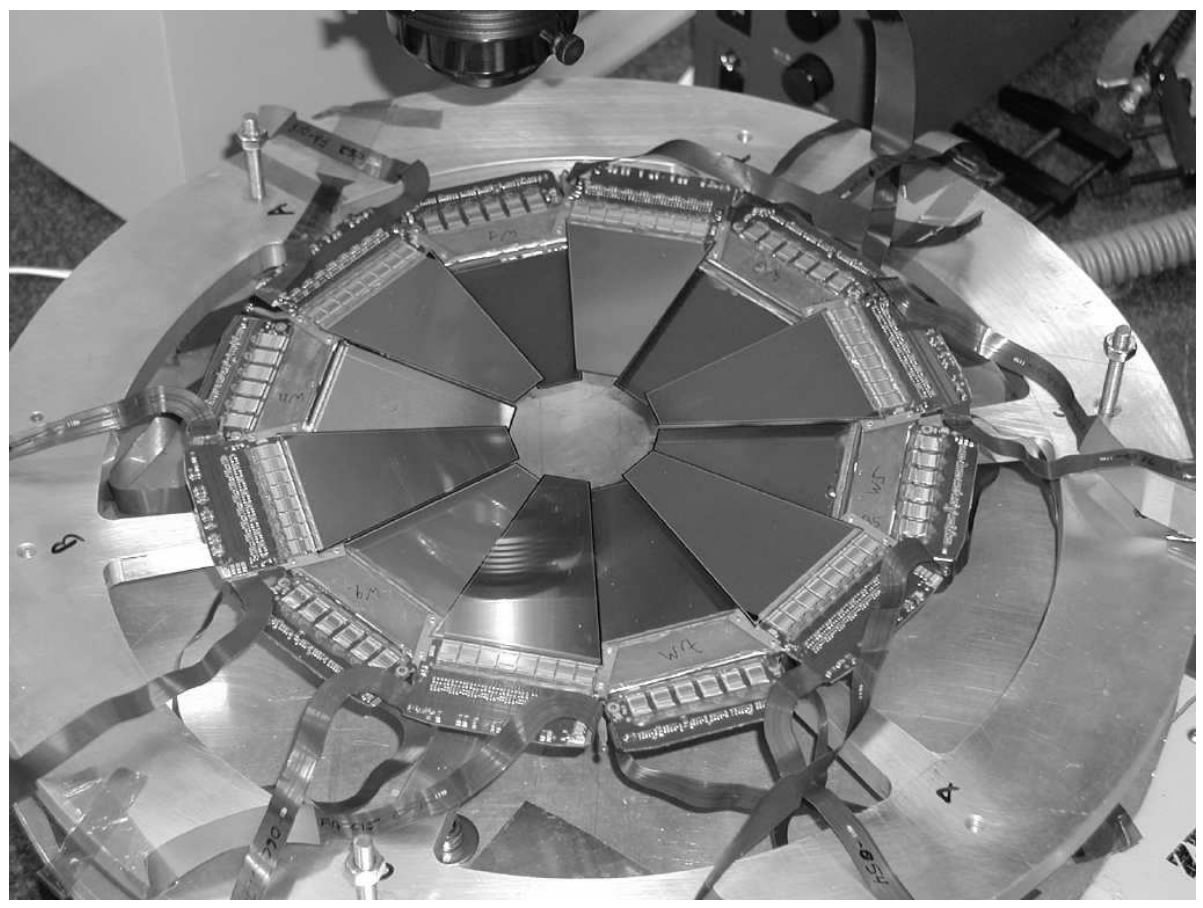

Fig. 42. First prototype F-disk assembly.

alignment holes to determine the positions of the disks as well as the wedges. After the two sides were laminated together, they were resurveyed for flatness and precision of the locating features on the two sides.

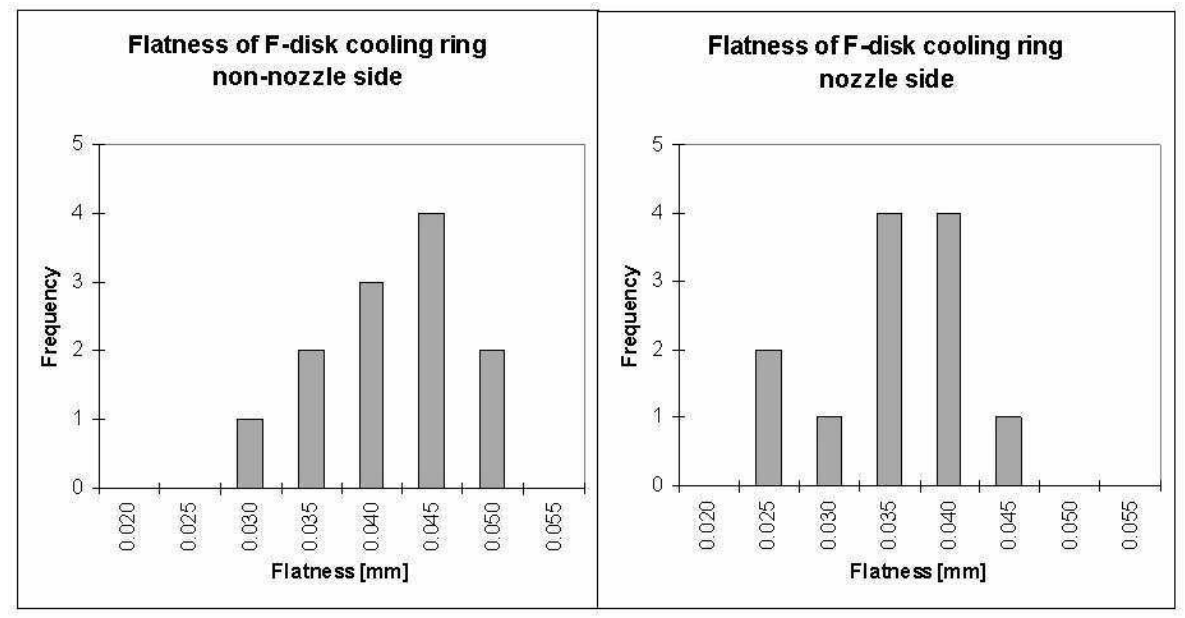

Fig. 43. Flatness distributions of beryllium ring halves used in F-disks.

The rest of the assembly was performed on a base plate with a center pin as a primary reference. Threaded studs, on which the wedges would be mounted, were inserted and then surveyed with respect to the alignment holes on the ring. The posts that position the disk on either the barrels or within the endring assembly were then mounted and inspected. Sapphire alignment balls, measuring $3 \mathrm{~mm}$ in diameter, were mounted on arms made from scrap beryl- 
lium and attached to the cooling ring. The balls were located in the space between two adjacent wedges near the 2, 4, 8, 10, and 12 o'clock positions, $12.5 \mathrm{~mm}$ radially outward from the cooling ring. After complete assembly, the rings were leak checked and surveyed for mechanical precision.

\subsubsection{Installation of Wedges on Rings}

Before the wedges were mounted on rings, the bias bonds on the corners of the sensor were encapsulated for protection. This encapsulation was done by hand, using a syringe to apply the encapsulant. The encapsulant was heat cured for an hour.

During the assembly of the first F-disk, the procedure was to also encapsulate all other bonds on the sensor. Each qualified wedge was retested immediately before placement on the ring. Leakage current tests indicated that there was some problem with the interaction of the encapsulant with the double-sided detector so this step was eliminated during assembly of the remaining disks.

The wedges were mounted on the rings using the same fixtures as for the ring assembly. This assembly was mounted on a CMM for precise alignment of the wedges. Each wedge has four mounting holes in the beryllium substructure which supports the HDI. These four holes engage studs mounted on the ring. The design clearance between the studs on the rings and the holes in the beryllium mounting plates of the wedges was $100 \mu \mathrm{m}$, allowing for adjustment of the wedge positions. The wedges had thermal grease applied to the cover layer over the jumper on the HDI where the mechanical contact occurs with the ring and good thermal contact needs to be ensured. The grease improves the heat flow through this joint by a factor of two. All six odd numbered wedges were installed on a ring and then the six were aligned relative to the ring mounting holes and center pin of the fixture. Figure 44 shows a partially populated ring assembly on the CMM.

Each wedge was aligned using the same two sensor fiducial marks used for gluing the sensor to the HDI. To move the wedge for alignment, two nuts were loosened, and the wedge was carefully pried to change position. This process was repeated until the fiducial positions matched the predetermined positions in $x$ and $y$ to within $10 \mu \mathrm{m}$. Once a wedge was positioned correctly, the nuts were tightened to hold it in place. This process was very difficult, and about $7 \%$ of the wedges were damaged and had to be replaced. After alignment, a final survey was done to record the wedge locations and survey ball locations relative to the ring reference holes.

Once the first six wedges were aligned on the ring, it was tested electrically to make sure no bonds had been damaged and that the wedges were still working correctly. The disk was carefully flipped over to mount the even numbered 


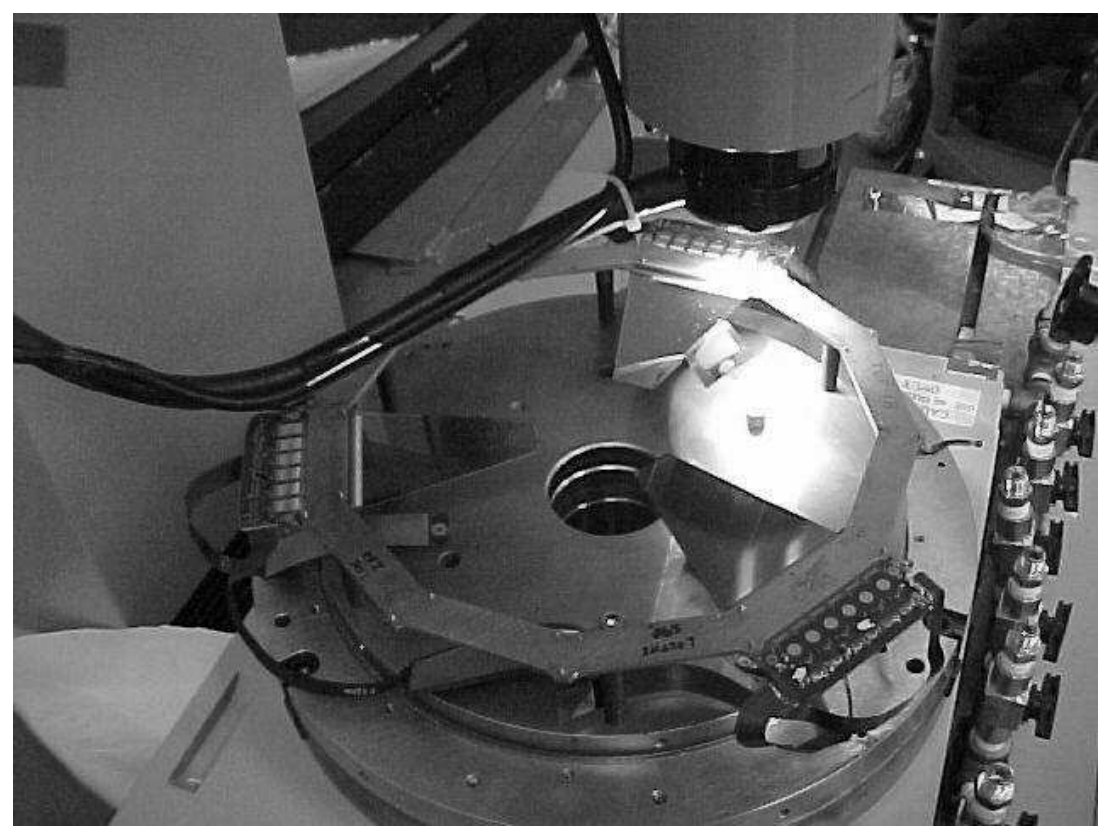

Fig. 44. Photograph of the beryllium F-ring with 3 wedges mounted on it.

wedges. The same mounting and alignment procedure was followed for the even numbered wedges. After all wedges were mounted and aligned on the ring, the disk was again electrically tested to ensure proper function.

Working disks were dismounted from the CMM disk assembly fixture and placed in individual boxes. These boxes were made from static free Plexiglas and included grounding straps for extra static protection. The disks were stored in a dry box until they were ready for mating with a barrel or with another end-disk. For the disks mated with barrels, a dimpled $0.025 \mathrm{~mm}$ polyimide foil was carefully mounted over the posts to try to minimize heat transfer from the disks to the barrel detectors mounted close by. The HDI tails were carefully dressed into a pinwheel design to hold them during mating.

\subsection{Mating of Barrels to Disks and End-disk Assemblies}

The final assembly step consisted of mating F-disks either to barrels or to one another to form the end-disk assemblies, followed by final dressing of cables and cooling tubes.

The mating of barrels to disks utilized a fixture consisting of a rotating Plexiglas disk from which the F-disk was suspended and a lifting table on which the barrel was located. The lifting table was initially used to transfer the F-disk from its storage box onto the hangers which suspended it from the Plexiglas disk. Additional locating stops limited the movement of the F-disk relative to the Plexiglas disk. The Plexiglas disk was then rotated to achieve the ap- 
propriate orientation relative to the barrel, and the barrel lifted into place under the F-disk until the posts extending from the F-disk ring engaged the mounting holes in the barrel bulkhead. The relatively unconstrained state of the F-disk allowed it settle into place without stress and provided visual feedback when it was not seating correctly. The barrel was raised until the F-disk assembly was lifted off the hangers before nuts were installed to secure the disk to the barrel. Finally, the F-disk HDI tails were dressed to the carbon fiber cable strain relief ring on the barrel and the assembly stored in a clean, dry environment until installation in the support cylinders.

The end-disk assemblies consist of sets of three F-disks mounted to one another. Because the spacing of these disks is not tight, it was possible to put these assemblies together without special tooling. The central disks have mounting posts on both sides with $5 \mathrm{~mm}$ ruby balls at the ends, while the inner and outer disks have posts only on the side facing the center disk with conical receptacles which accept the $5 \mathrm{~mm}$ ruby balls. The central disk was left mounted in its storage box while the inner disk was manually installed on it. Structural epoxy was used on the ruby balls to permanently bond the rings together. Spring clips were used at each post-ball-post interface to ensure proper seating of the balls while the epoxy cured. The assembly was then flipped over and the outer disk added in a similar fashion. Cables and cooling tubes were dressed and the assembly stored in a clean, dry environment until installation in the support cylinders. 


\section{Installation of the Detector}

The SMT detector was built at the Fermilab Silicon Detector Facility and then transported about $3 \mathrm{~km}$ to the D0 collision hall. The installation of the barrels and F-disks within the bore of the CFT, shown in Fig. 45, was completed in December 2000. The final H-disk was installed in early February 2001, and about $20 \%$ of the electronics were in place for the beginning of Run II in March.

Detailed descriptions of the transportation and installation of the barrel detector are given in the following section.

\subsection{Transportation and Installation of the Barrel Detector}

The assembled detector halves were mounted inside transportation boxes for the drive to D0, as well as the trip from the loading bay to the center of the D0 detector.

The cushioned box incorporated several features for various portions of the transportation and installation journey. The main components of the box were a base plate with a rail system mounted on it, a 4-sided enclosure (cover), a cable tray assembly mounted under the base-plate, and a set of rollers to allow the box to be slid along a trolley system in the collision hall. In addition, a cart with air suspension was used during the trip from the assembly lab to the loading dock at the experimental hall to reduce accelerations to less than $5 g$.

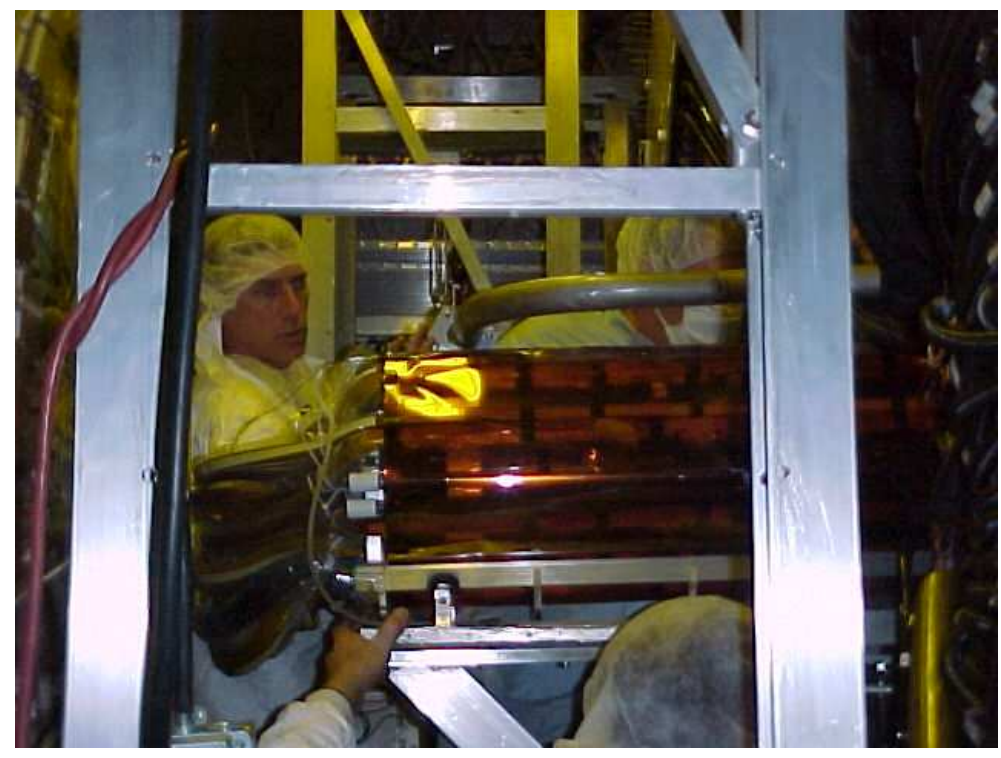

Fig. 45. Installation of the silicon detector in the CFT. 


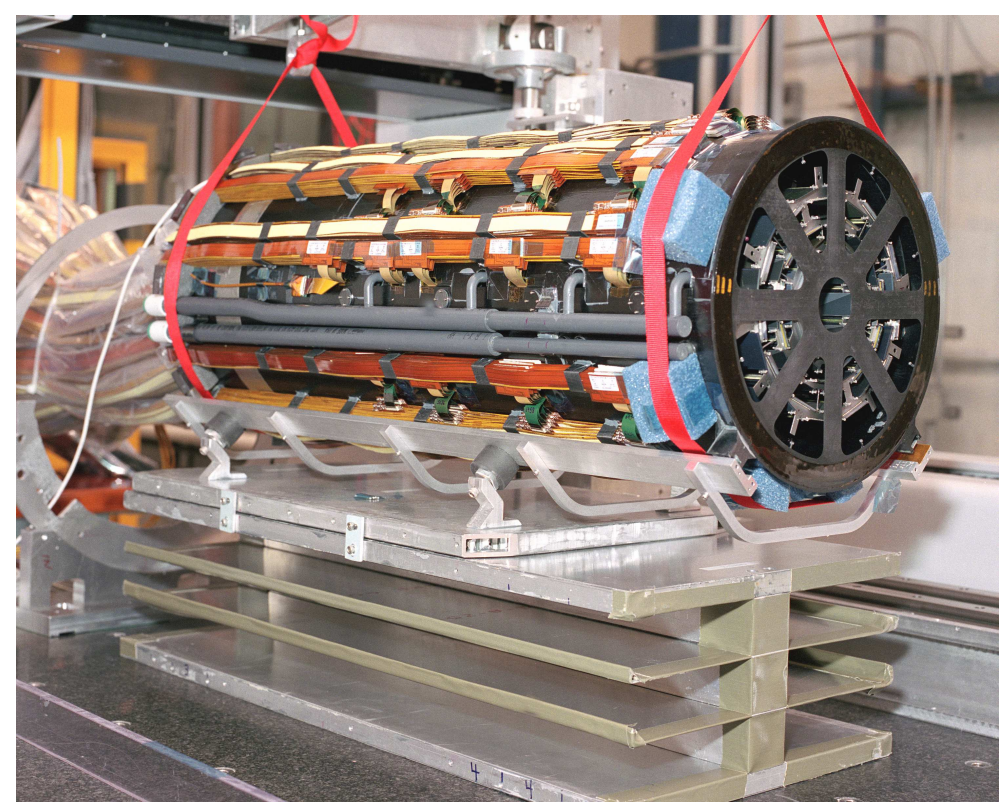

Fig. 46. Installation of the silicon detector on the transportation rail.

The first stage of the installation journey involved transferring the completed detector assemblies from the rotating fixtures used for barrel and disk installation and cabling onto the rail system mounted to the base plate of the transportation box. This was done using the slide and ball screw mechanism which was used to install the barrel and disk assemblies into the support cylinder. Straps were run around the cylinder and the cylinder was slowly lifted and released from the rotating support frame, which was then removed from under the cylinder. The transport box base, with cable tray, was then slid under the cylinder and the cylinder lowered onto the rails (Fig. 46). The cylinder has "feet" at the $z=0$ end at $\pm 45^{\circ}$ which are the final supports from the CFT inner barrel. At the outer end, temporary feet were screwed to the outer membrane of the support cylinder. Once lowered onto the rails, small clamps were used locally at each foot to secure the cylinder to the rail system. The rail system was coupled to the base plate of the box through rubber mounts to provide some isolation from impact loading during movement, as well as to allow for small alignment corrections when these rails were mated to the pre-existing rail system inside the fiber tracker.

The 4-sided cover was installed next. This provided mechanical protection for the assembly as well as a semi-sealed volume for purge gas. The outer end of the box was left open where the cables exited. The cables were then dressed into the cable tray assembly under the box, then the box assembly was sealed from the bottom of the cable tray assembly to the box cover to provide a sealed purge gas volume. A nitrogen purge was provided during the drive. This was important as the detector was moved during the winter and condensation onto the detector was possible after arrival at the collision hall. Finally, a set of brackets was installed which coupled to the base plate, ran outside the box 
cover, and terminated above the center of gravity of the assembly with rollers for the trolley system used in the collision hall.

The completed package was loaded onto an air-ride cart designed to limit impact loading to less than $5 g$ during the truck ride to the collision hall. Several test runs were made using a dead weight on the cart to simulate the silicon load. Accelerometers attached to fixed points on the cart routinely registered loads in excess of $5 g$ and occasionally above $10 g$ when the truck hit small bumps in the road. However, the mock silicon assembly was insulated such that loads were always below the $5 g$ threshold. During the transportation of the two detector halves, accelerometers were placed on the silicon enclosure to monitor the device in all three directions; none of the $5 g$ sensors tripped during transportation.

Upon arrival at the collision hall, the detector halves were lifted by crane directly from the rear of the truck to the trolley system. A length of pipe was slid through the trolley system rollers on the transportation box and stops were installed to prevent the box from moving along the pipe. The pipe was then picked up by the crane and the assembly lifted from the air ride cart and "flown" to the detector area. The trolley pipe traveling with the silicon was joined to the fixed section running from the outside of the detector into the calorimeter gap. The detector was then rolled along the trolley system into the area just outside the calorimeters. At this point, the cables were unpacked and hung from additional carts on the trolley system. The box cover was also removed at this point. The silicon was moved along the remainder of the trolley path into the calorimeter gap where the assembly was lowered onto a table mounted onto the calorimeter.

At this stage, the silicon was still sitting on the rails mounted to the transportation box base plate. The base plate was slid by hand until the rails engaged the ends of the rail system inside the inner barrel of the fiber tracker. The final step was to slide the detector along the rails into its final position, at which point the outer end of the detector was secured in place with a set of nuts engaged on titanium studs at the end of the fiber tracker.

After removal of the installation equipment, final connections were made to the cooling system and cables. After connection of each cable, a functionality test was performed to ensure a good connection.

The cabling of the more than 15000 connections and the installation of all electronics were completely finished in May 2001. Problems during the commissioning mainly concerned power supplies and the distribution of the operating and high voltages. 


\section{Performance and Lifetime}

After its installation 2001, the SMT has been successfully operating for more than eight years. The detector is performing very well, providing good tracking and vertexing for the D0 experiment.

The signal-to-noise ratio was measured to be between 10 and 15 depending on the module type. The signal is defined as the cluster charge given by a minimum ionizing particle including a correction for the incident angle, and the noise as the rms of the pedestal distributions. The noise consists of intrinsic, random noise coming from the front-end of the SVXIIe chip, and environmental, coherent noise. The signal-to-noise ratio calculated only from random noise is between 12 and 18. Gains vary among detector types with the n-sides 5-15\% lower than the p-sides due to the larger load capacitance. Pulse height information from the SVXIIe is used to calculate cluster centroids and can also be used for $d E / d x$ tagging of low momentum tracks. Figure 47 shows $d E / d x$ distributions after corrections for gain and incident angle are made.

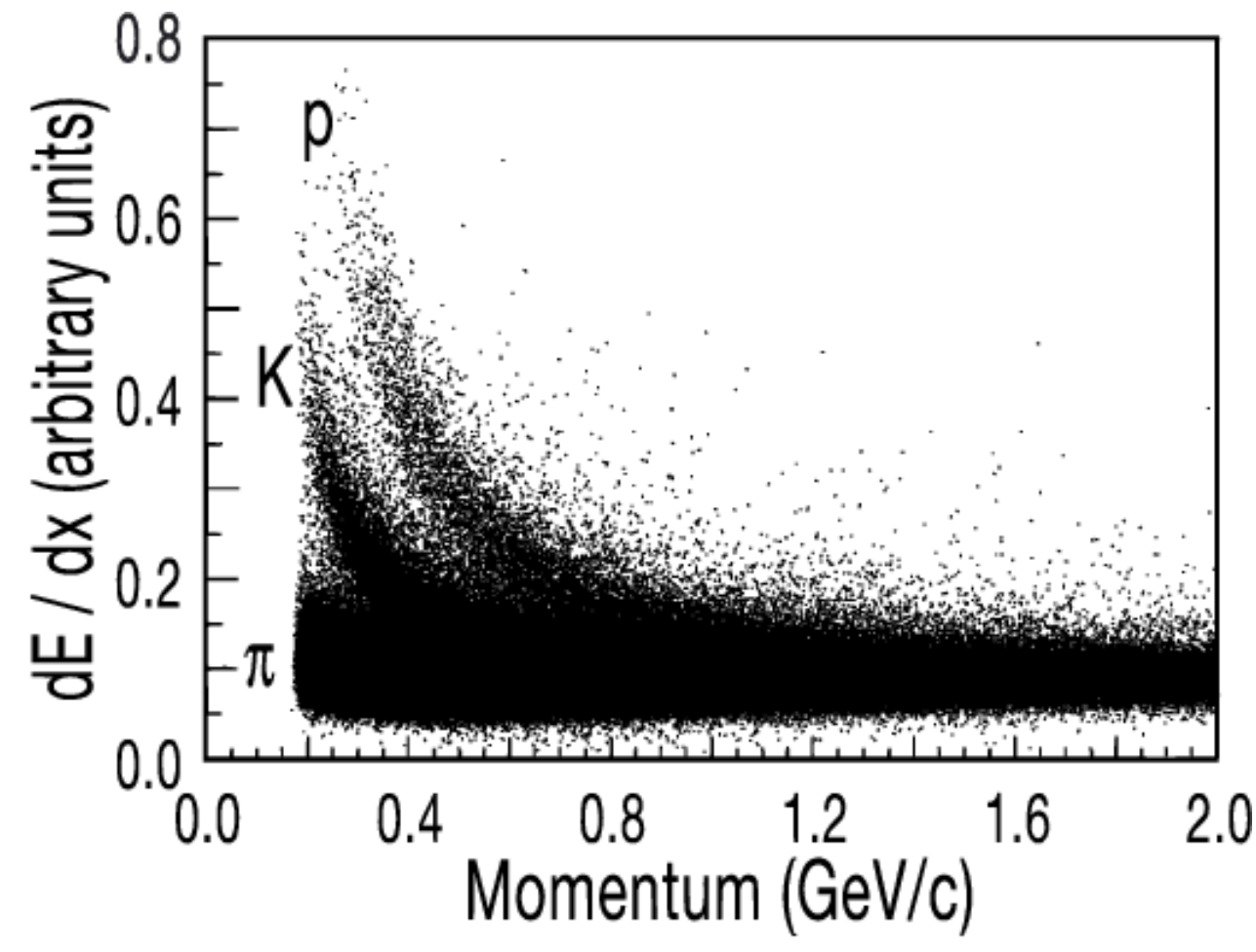

Fig. 47. Distribution of energy loss for a kaon-enriched sample of tracks showing $\pi, K$, and proton bands.

Figure 48 shows the impact parameter distribution for tracks with respect to the primary vertex. Only charged particles with transverse momentum larger than $3 \mathrm{GeV} / c$ are included in the plot. The only a lignment in this case is the geometrical alignment described in Sec. 8. The Gaussian fit has a width 
of $60 \mu \mathrm{m}$ to which the beam size contributes 30 to $40 \mu \mathrm{m}$. After an alignment procedure where both the hits in the SMT and CFT and the primary vertex are used to constrain the fit, the impact parameter resolution is much improved and corresponds roughly to what is expected from simulation studies. The impact parameter as a function of transverse momentum of charged particles is shown both for data and simulation in Fig. 49. Figure 50 shows a "photometric" picture in $x / y$ view of the SMT. The precise tracking allows reconstruction of the $x y$ coordinates of photon conversions in the detector material. Different layers of the SMT are clearly seen in this picture.

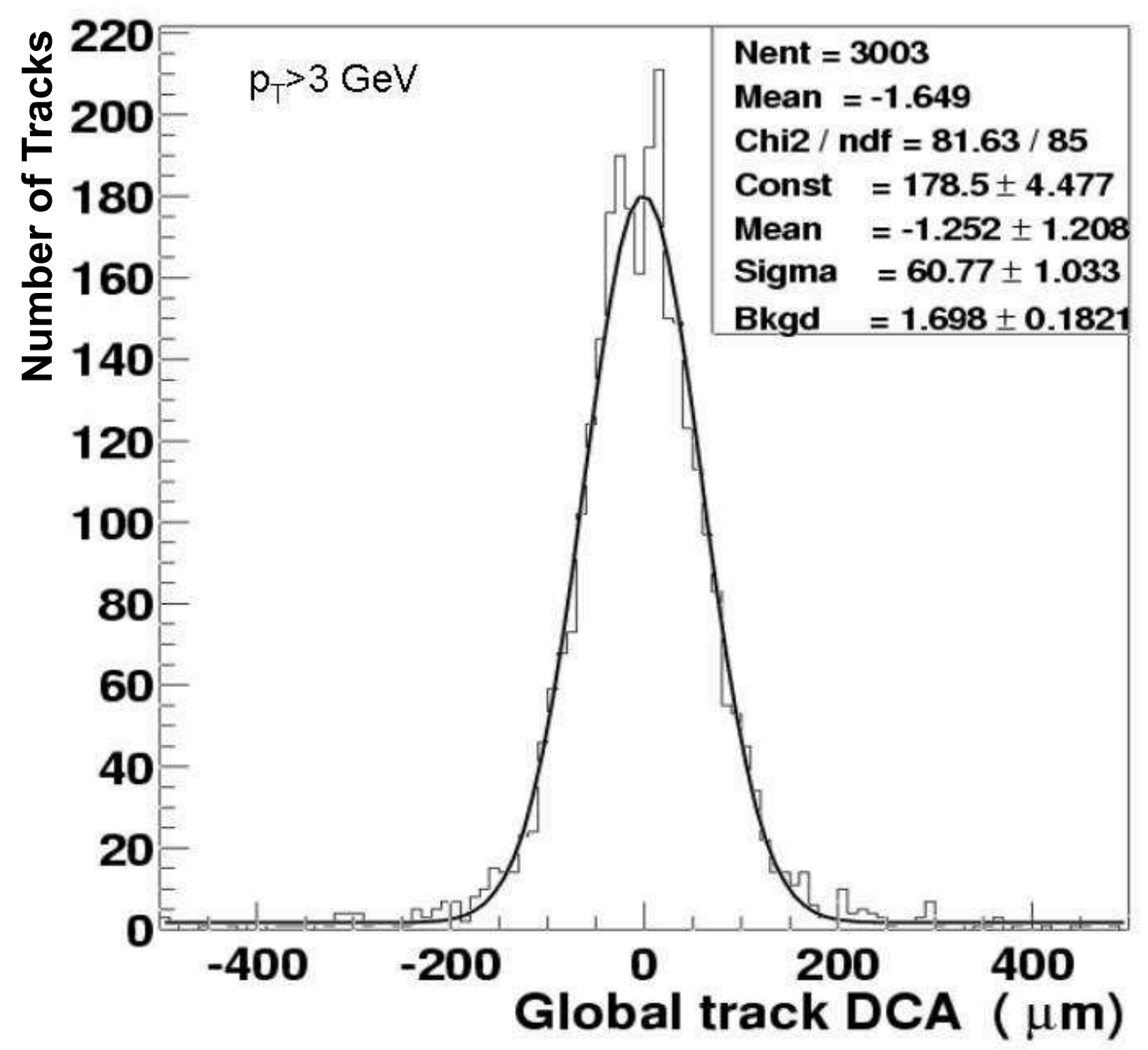

Fig. 48. The impact parameter of charged tracks with transverse momentum large than $3 \mathrm{GeV} / c$ with respect the primary vertex.

Operational difficulties have in many cases been peripheral to the silicon detector itself. These include latchup of operational amplifiers on the interface boards, low-voltage power supply failures, and high leakage currents in the high-voltage distribution boxes. However, the detectors themselves have suffered from some important problems and failures which are discussed in the following sections. In the next section, some very noisy modules are described; in Sec.10.2, problems with the readout system are discussed, and finally the 


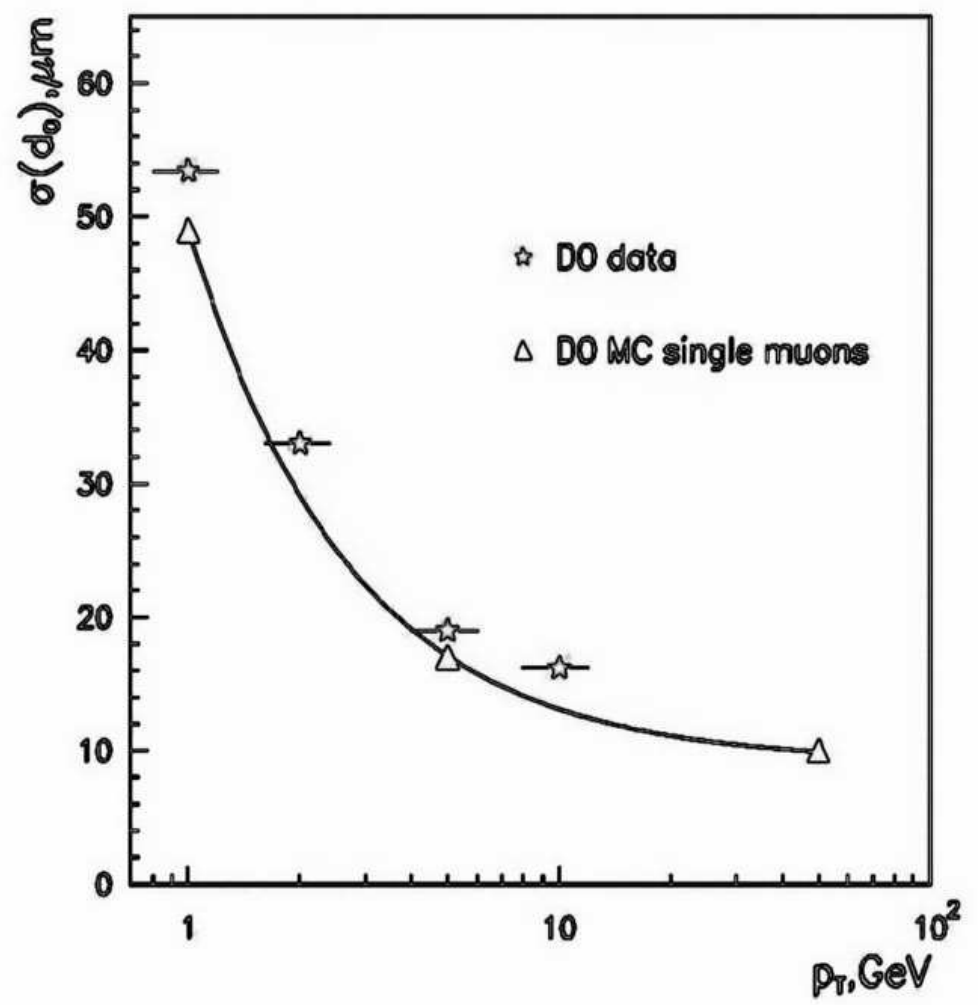

Fig. 49. The rms of the impact parameter distribution as a function of the transverse momentum of charged particles is shown both for data and simulations.

aging problem due to radiation damages and a life time estimate are presented.

\subsection{Noise in the F-wedges}

A serious detector feature is the so-called "grassy noise" shown in Fig. 51, which is confined to the Micron-supplied F-disk detectors which correspond to $75 \%$ of the F-disk sensors. This noise is characterized by large charge spikes which cover 10-20 strips, and it occurs in about $20 \%$ of the events for affected devices. Leakage currents typically rise to greater than $100 \mathrm{~mA}$ within $1 \mathrm{~h}$ of turn-on at the beginning of a store. The only major change in the grassy noise was its onset about two months after the start of the run. Some of the devices continue to slowly increase their current draw. The current increases about $100 \mathrm{~mA}$ after detectors are biased; these increases for a store which appear to be charge-up effects with time constants of tens of minutes. 


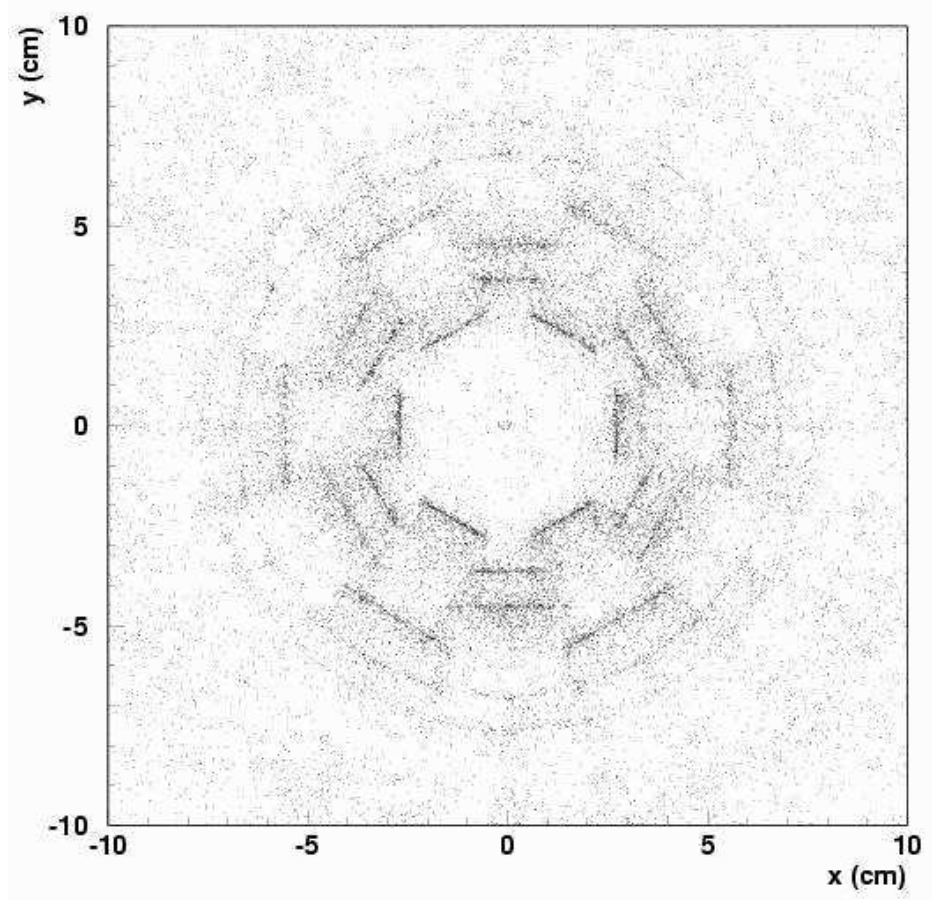

Fig. 50. A "photometric" picture of the SMT produced by plotting reconstructed coordinates of $\gamma$-conversions.

\subsection{Performance of the Readout System}

At the start-up in 2001, $95 \%$ of the ladders, $96 \%$ of the F-wedges and $87 \%$ of the $\mathrm{H}$-wedges were working. Over time, these numbers have varied, and Fig. 52 shows the number of disabled modules from 2003 to 2010. The gaps in the figure correspond to machine shutdowns. During each shutdown period, several modules have been recovered by repair work to the external electronics and cabling. However, most of the disabled modules have problems in a inaccessible area, i.e., in the SVXIIe chips, in the HDIs, or in the low mass cables.

Many of the problems with the chips arise because of an unwanted increase in the supply currents. This could happen if the SVXIIe stands idle for time periods measured in hundreds of seconds; some of the supply currents increase by as much as a factor of 5 . The cause of this has been traced to the use of dynamic CMOS memory in the chip. Figure 15] shows part of the schematic of the SVXIIe. At the far right of the schematic, two capacitors are labeled Dout1 and Dout2. These capacitors are examples of the dynamic CMOS memory used in the SVXIIe. In one half of a cycle, the capacitors are either set to a voltage or to zero, and they are read in the second half of the cycle. However, if there is no clock signal, leakage currents slowly charge the capacitors. Since these devices drive CMOS gates, the intermediate voltage will turn on both 


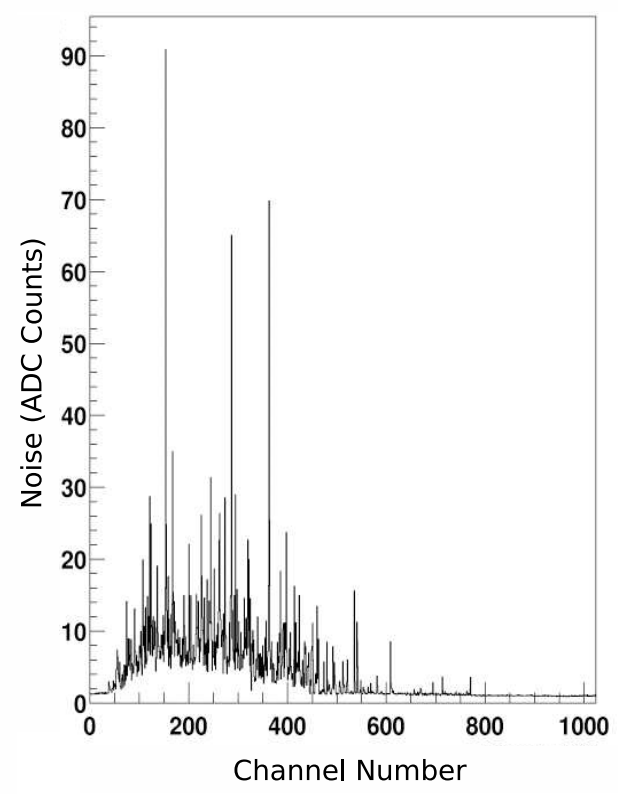

Fig. 51. Example of the grassy noise seen in the Micron-supplied F-disk detectors. Ideally, the entire plot would look like the region above channel 800 .

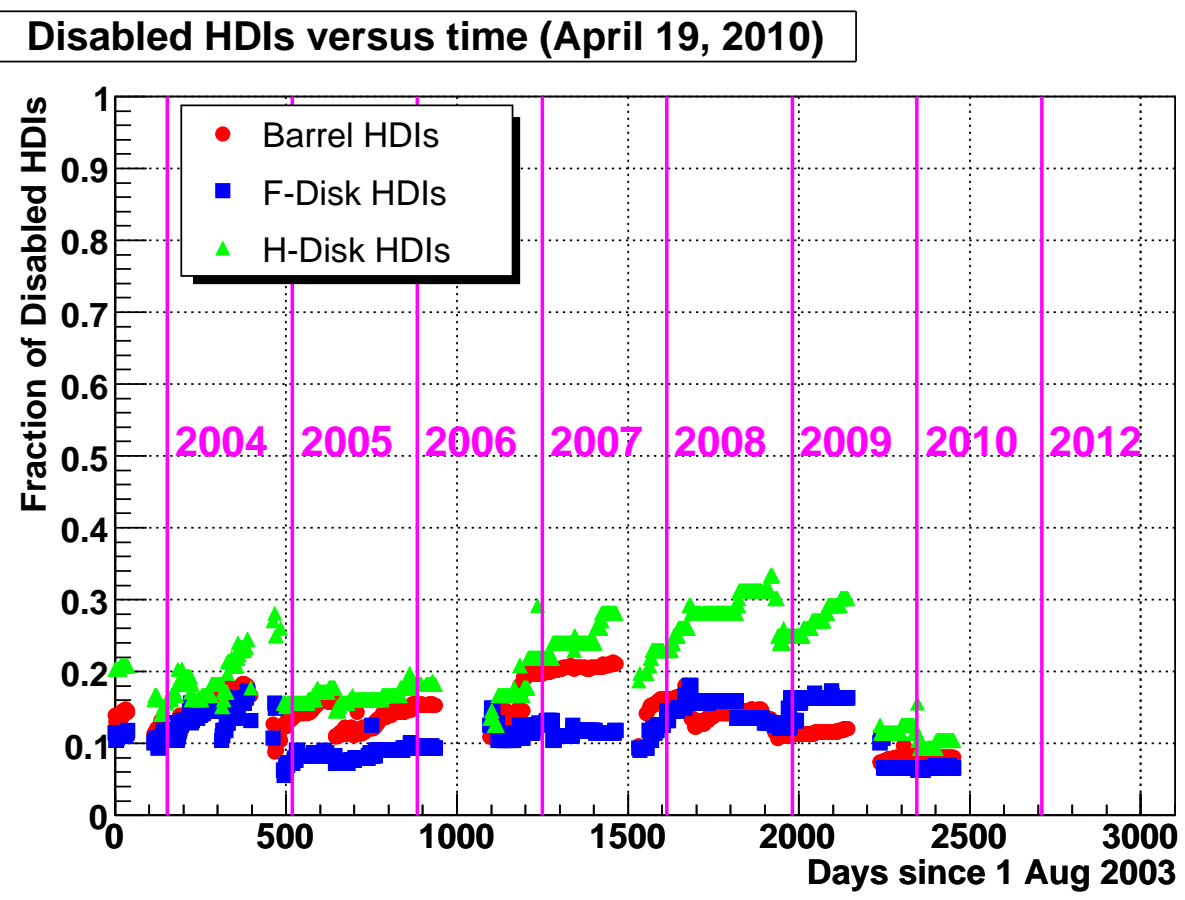

Fig. 52. The fraction of disabled HDIs as a function of time.

the $\mathrm{n}$ and $\mathrm{p}$ transistors, and the current will increase. This feature can lead to excessive chip currents and can evidently cause power wire-bond failure. A special signal is installed that cycles the SVXIIe every few seconds if there is 
no beam to avoid the increasing current. However, in one incident, the loss of the clock signal to the detector apparently caused the failure of several HDIs due to power wire-bond failures. After that, a special watch-dog timer is under development that will shut off the chip power if the special signal fails.

One chip not working on an HDI due to a power wire-bond failure means that the whole HDI is not functional and has to be disabled. However, it has been possible to recover most of the data from these HDIs. Since it is impossible to get to the HDIs, the repair was done from outside the detector. The wire bond that failed was for the digital power. The digital bus lines have protection diodes to the digital power bus inside the chip, and there is a small resistor in series with the diode to limit the current. It turned out to be possible to power the chip from the bus lines. However, the resistor prevents enough power to do an entire readout, but with the chip set to a very high threshold and in zero-suppressed mode, the entire string of chips except for the one that failed was recovered.

Since there is a diode drop in the protection diodes in the chip, the bus voltage has to run near $5 \mathrm{~V}$. This is higher than the output of the standard bus drivers so new adapter cards that provided the higher voltages were produced. These new adapter cards were installed where needed in the 2007 and 2008 shutdowns.

Another major failure appears to be from connector failures. One H-disk unit on each side was removed to make room for a new inner layer Ref. [7] in 2006. The two H-disks had numerous failures before being removed, and were investigated after the removal. It turned out that 14 out of 24 bad HDIs had no failures during extensive bench testing. The flexible tail is designed to take care of slight cable mis-alignments. However, in the case of the H-disk, the tail was over-constrained by a carbon fiber structure. If the low mass cable was installed at a slight angle in the plane of the cable, a small torque was placed on the cable, which was evidently enough to cause the connectors to open.

Eight of the H-disk HDIs that had actually failed were further inspected. Six had a broken wire bond in the priority passing line. The other two had unknown failures. The cause of the wire bond failures appears to be the encapsulant. The encapsulant had re-liquefied and the re-liquefaction started at the surface of the HDI. The H-disks are mounted vertically so when the encapsulant released from the HDI, any stress would be split between the one bond wire going across the chips and the 19 wires going along the chips. The evidence suggests that the stress on the one wire was too great and it failed. The cause of the re-liquefaction of the encapsulant is not known.

In addition to the problems discussed above, the modules suffered from some recoverable errors. The most common are a failure in the ring counter that 
controls the pipeline and a failure to download properly. The ring counter error is a design oversight in the chip. Its symptom is that in digitize mode it connects a DC voltage to the comparator input rather than the output of a capacitor. The only solution to this is to reset the pipeline counters. This error is induced by electrical noise, and the noise from a digitization and readout frequently causes this error. To overcome this problem, the pipeline counters are reset after every readout. This also resets the pipeline, so any triggers in the pipeline are lost.

\subsection{Radiation Damage}

The lifetime of the SMT is expected to be limited by noise due to microdischarge breakdown in the inner layers, as discussed in Sec. 5. The microdischarge effect depends on bias voltage and becomes unacceptable at approximately $150 \mathrm{~V}$. The bias voltage needed to deplete the detector modules changes with the radiation dose they have received. To predict the lifetime of the SMT, it is necessary to monitor the depletion voltage and the radiation dose. The inner layer of the inner four barrels consists of the DSDM silicon modules which were measured to be the most sensitive to radiation. In addition, the inner layer receives the highest radiation dose. Therefore, this study is concentrated on the DSDM silicon modules in the inner layer.

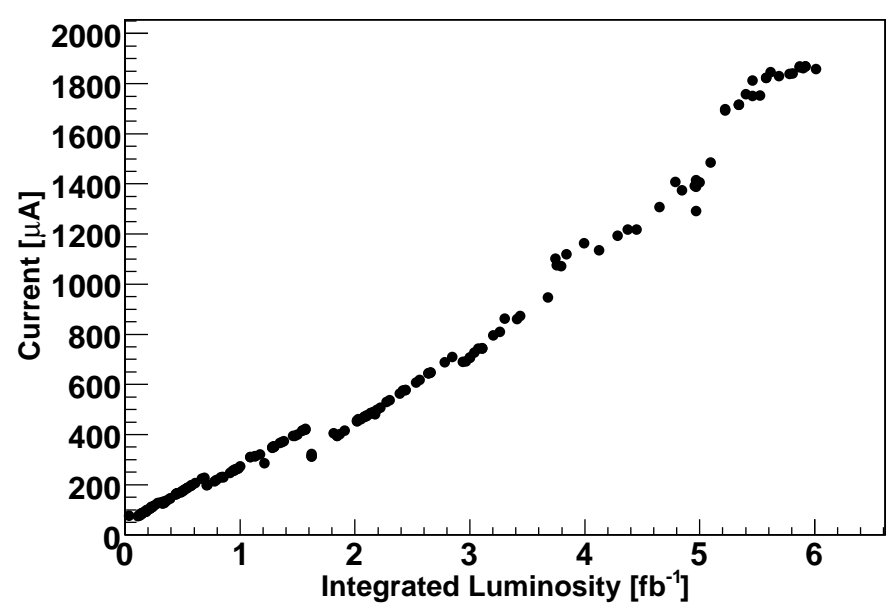

Fig. 53. Evolution of the leakage current normalized to $20^{\circ} \mathrm{C}$ with integrated luminosity.

The radiation dose received by a silicon module can be measured using the leakage current, which depends on flux according to the following formula:

$$
I=I_{0}+\alpha \cdot \Phi \cdot V
$$


where $I_{0}$ is the leakage current before irradiation, $\alpha$ is the radiation damage coefficient, $\Phi$ is the particle flux, and $V$ is the detector volume. The coefficient $\alpha$ depends on the particle type, temperature, and time [17,28]. The particle flux is proportional to the integrated luminosity delivered by the Tevatron. Therefore the dependence of the leakage current on the integrated luminosity is also expected to be linear. In Fig. 53 the current is plotted versus the integrated luminosity for four DSDM silicon modules. It can be seen in the figure that there is an almost linear dependence. The drop in the leakage current at for example the integrated luminosity $0.6 \mathrm{fb}^{-1}$ occurred because the SMT was warmed up to $15^{\circ} \mathrm{C}$ for more than one month, and the annealing processes accelerated. The inner silicon layer has two sub-layers: the inner sub-layer at radius $27.15 \mathrm{~mm}$ and the outer sub-layer at radius $36.45 \mathrm{~mm}$. The radiation dose collected by the outer sub-layer is about 1.6 times lower than the one at the inner sub-layer. Measurements of several silicon modules in the inner layer gave a normalized particle flux from $4.0 \times 10^{12}$ to $5.3 \times 10^{12}$ particles $/ \mathrm{cm}^{2} \cdot \mathrm{fb}^{-1}$, taking annealing parameters from [28] and an asymptotic radiation damage constant of $3 \times 10^{-17} \mathrm{~A} \cdot \mathrm{cm}^{-1}$. The spread reflects the accuracy of the method as well as possible non-uniformity of the radiation dose.

The silicon detector bulk material at D0 is slightly n-doped. Under radiation, however, donor states are removed and acceptor states created, eventually leading to a change from positive to negative space charge, i.e. the silicon bulk goes from being n-doped to becoming p-doped. This phenomenon is referred to as type inversion and has been confirmed by many experiments. It was predicted that the type inversion at the D0 silicon bulk material would happen when the integrated normalized particle flux reached approximately $5 \times 10^{12}$ particles $/ \mathrm{cm}^{2}$. At the point when the Tevatron had delivered about $1 \mathrm{fb}^{-1}$, the inner layer was expected to have reached the type inversion point, where the depletion voltage is minimal. The following sections provide a description of depletion voltage measurements.

\subsubsection{Noise Level on the $n$-side}

While the silicon bulk material is n-doped, the noise of the n-side strips is large and constant at all voltages below the depletion voltage, and is reduced significantly when depletion is reached. Thus studying the n-side noise as a function of bias voltage can be used to determine the depletion voltage.

In a bias voltage scan, 11 runs are taken, with the high voltage settings varied from 0 to $100 \%$ in steps of $10 \%$. The bias voltage scans were performed with and without beams in the Tevatron. The beam presence does not affect the measured depletion voltage values.

The DSDM and DS sensors show very different noise behavior as a function 
of bias voltage. For that reason, different procedures are used for DSDM and DS devices.

The DSDM devices show a rather unexpected noise behavior as a function of bias voltage (see Fig. 54). There is no abrupt decrease in the noise level on the n-side when the depletion voltage is reached. Instead, the noise is decreasing rather monotonically with increasing bias voltage. For some HDIs, a small kink in the noise can be seen at a certain bias voltage, and the position of this kink changes as a function of the radiation dose. This kink is interpreted as an indication of the depletion voltage.
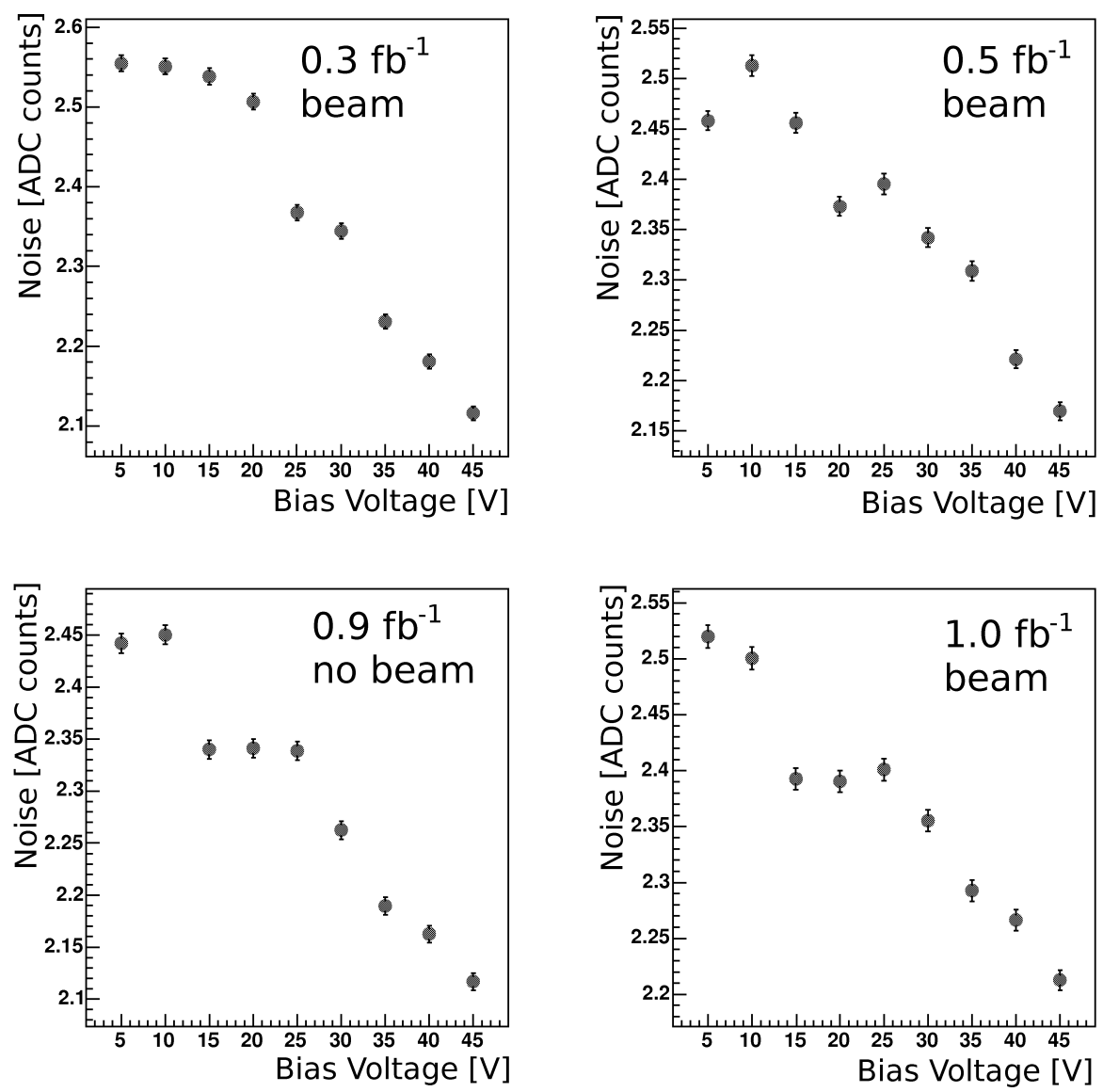

Fig. 54. Distribution of noise level on the n-side as a function of bias voltage for a DSDM silicon module installed in the D0 detector. The bias voltage scans at different integrated luminosities are shown. The depletion voltage at $0.3 \mathrm{fb}^{-1}$ is $25 \mathrm{~V}$, at $0.5 \mathrm{fb}^{-1} 20 \mathrm{~V}$, and at 0.9 and $1.0 \mathrm{fb}^{-1} 15 \mathrm{~V}$.

The n-side noise was measured with a non-irradiated test DSDM module and abnormal behavior was not observed. This measurement indicates that the abnormal noise behavior is caused by the irradiation, and the comparison with the DS modules shows that the radiation changes some properties of the PECVD layer. 
Most of the DS devices show the expected noise behavior as a function of bias voltage (see Fig. 555). At low voltages the noise is large, and rapidly decreases to a stable and lower level as soon as the bias voltage reaches the depletion voltage. There is some indication of noise increase at bias voltages higher than the depletion voltage after higher radiation doses.
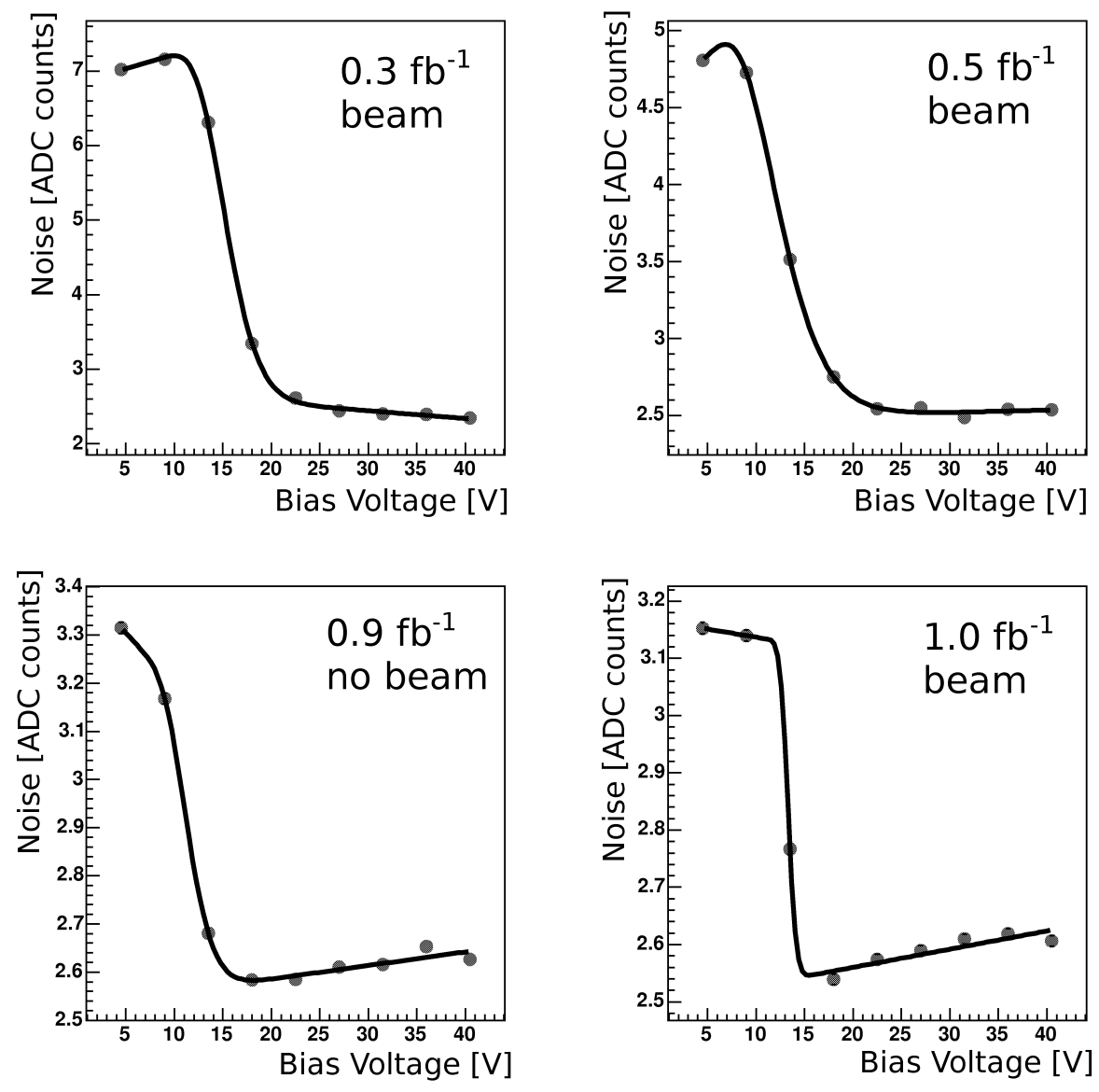

Fig. 55. Distribution of noise level on the n-side as a function of bias voltage for a DS silicon module installed in the D0 detector. The bias voltage scans at different integrated luminosities are shown.

\subsubsection{Charge Collection Efficiency}

Another method for determining the depletion voltage uses the dependence of charge collection efficiency on bias voltage. For the silicon detectors with n-doped bulk material, the charge collection efficiency measured on the p-side increases with bias voltage and reaches its maximum at full depletion. It is possible that there is some additional small increase after that, for example, due to change of the charge collection time.

This method requires bias voltage scans with tracks in the detector. A special 
algorithm has been developed for cluster reconstruction on the ladder under study in the vicinity of the expected track position. To determine the charge collection efficiency, the cluster charge is measured. The bias voltage where the charge collection efficiency reaches $95 \%$ of its asymptotic value has been identified as the depletion voltage.

The depletion voltage measured using the charge collection efficiency shows agreement with the noise measurements.

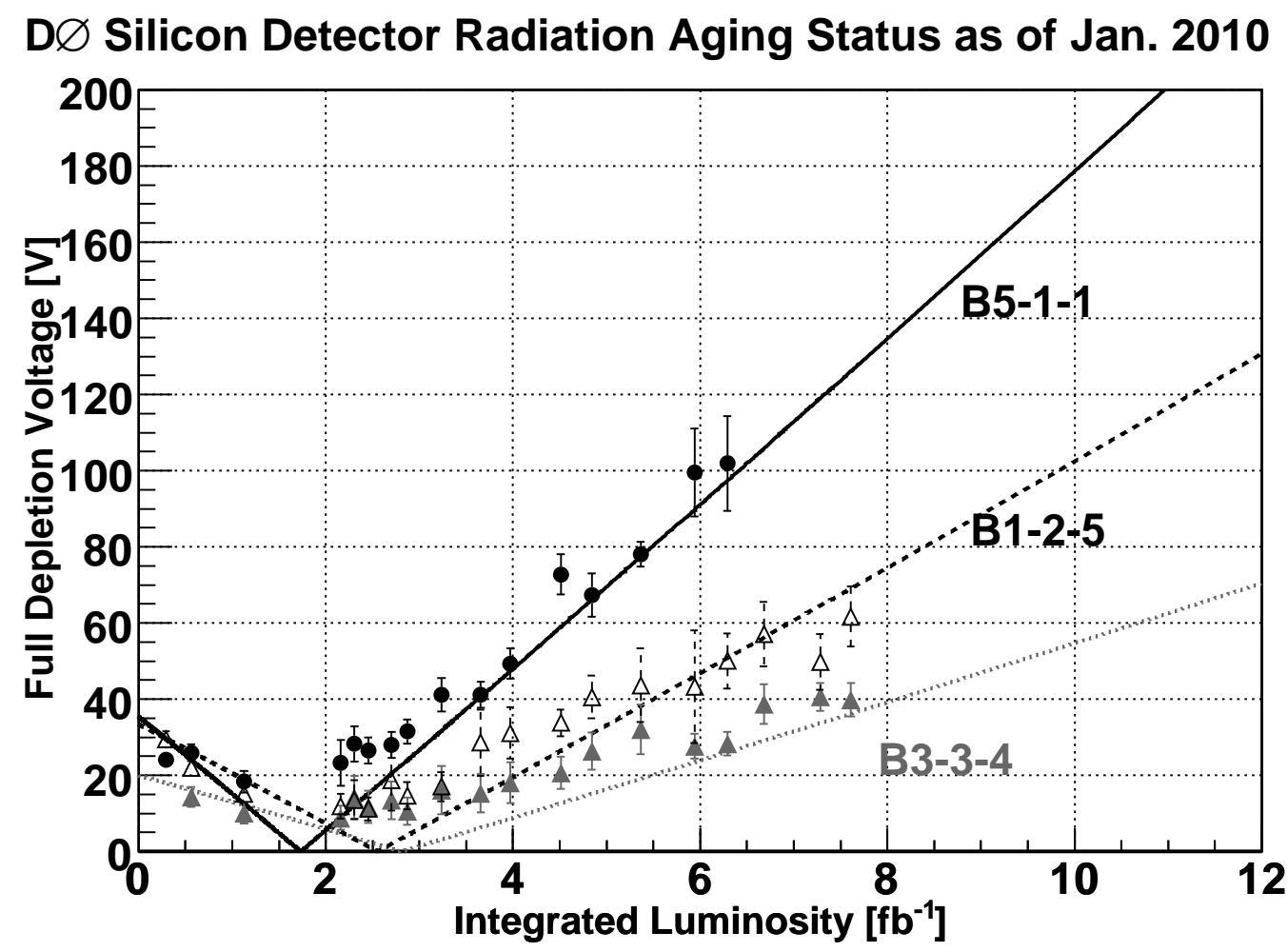

Fig. 56. Depletion voltage for silicon modules installed in the D0 detector for different radiation doses. The DSDM module is located at a radial distance from the beam of $2.7 \mathrm{~cm}$, the $\mathrm{SS}$ module at $3.6 \mathrm{~cm}$ and the DS module at a radial distance of $4.4 \mathrm{~cm}$. The lines represent theory predictions according to Ref. [17].

\subsubsection{Lifetime Estimate}

The measurements of the radiation dose and depletion voltage allow us to compare the behavior of the silicon sensors installed in SMT with those irradiated at the Radiation Damage Facility (RDF) at Fermilab. A comparison shows that the DSDM silicon modules irradiated at the RDF and those installed in the SMT have a different dependence of the depletion voltage on the radiation dose. The depletion voltage for the DSDM irradiated sensors increases much faster than for the DS silicon detectors tested at the RDF (see Fig 21). From our measurement see Fig. 56, the behavior of depletion voltage for the DSDM silicon modules installed in the SMT is similar to the behavior of the 
DS modules and is well described by standard parameterizations [17]. Assuming validity of DSDM detectors tests at RDF the discrepancy is probably due to slow annealing of charge trapped in the insulator layer between metal layers which was not measured in the RDF studies. Assuming this "normal" behavior continues, the depletion voltage for the DSDM silicon sensors at the inner layer is predicted reach values around $150 \mathrm{~V}$ at a delivered luminosity of $8-9 \mathrm{fb}^{-1}$. It is seen that the micro-discharge for these ladders increase at $110 \mathrm{~V}$ with the luminosity of $6.5 \mathrm{fb}^{-1}$, which could limit maximum operating voltage for these ladders to less than $150 \mathrm{~V}$. 


\section{Conclusion}

The SMT contains a large variety of component types. It holds six different types of sensors, one sensor type is double sided, double metal; two are double sided; and three are single sided. Nine different types of flex circuit carrying the readout chips and other passive electronic components were needed to accommodate the various sensors and readout geometries. This variety of components made the production of the detector challenging. Despite many difficulties, the SMT was successfully assembled and installed at the start of Tevatron Run II. The detector has worked well since then. However, due to radiation damage, the detector is aging and in order to compensate for this anticipated performance degradation and to improve the momentum resolution, a new inner barrel layer, called Layer 0 was installed in 2006. A description of layer 0 can be found in Ref. [7].

The SMT detector has been part of the D0 readout chain since the beginning of data taking in Run II, contributing to the almost two hundred interesting physics publications by the D0 collaboration.

\section{Acknowledgements}

We thank the staff at Fermilab and in particular at SiDet, the D0 mechanical and electrical support personnel and all our d0 collaborators for there support. Especially we would like thank George Ginther and Susan Blessing for their thorough reading of the draft and for giving countless useful comments and suggestions. We also acknowledge support from the DOE and NSF (USA); CEA and CNRS/IN2P3 (France); FASI, Rosatom and RFBR (Russia); CNPq, FAPERJ, FAPESP and FUNDUNESP (Brazil); DAE and DST (India); Colciencias (Colombia); CONACyT (Mexico); KRF and KOSEF (Korea); CONICET and UBACyT (Argentina); FOM (The Netherlands); STFC and the Royal Society (United Kingdom); MSMT and GACR (Czech Republic); CRC Program and NSERC (Canada); BMBF and DFG (Germany); SFI (Ireland); The Swedish Research Council (Sweden); and CAS and CNSF (China). 


\section{References}

[1] S. Abachi et al., Nucl. Instr. and Meth. A 338 (1994) 185.

[2] S. Abachi et al., Phys. Rev. Lett. 742632 (1995).

[3] See http;//www-d0.fnal.gov/d0_publications/ for a complete list of D0 publications.

[4] V. M. Abazov et al., Nucl. Instr. and Meth. Phys. Res. A 565 (2006) 463.

[5] V. M. Abazov et al., Nucl. Instr. and Meth. Phys. Res. A 552 (2005) 372 .

[6] T. Adams et al., The D0 Run II impact parameter trigger, arXiv:physics/0701195.

[7] R.Angstadt et al, The Layer 0 Inner Silicon Detector of the D0 Experiment, arXiv:0911.2522.

[8] The SVXII design Group,A Beginners Guide to the SVXII, FERMILAB-TM-1892.

[9] The MIL-1553B standard.

http://www.interfacebus.com/Design_Connector_1553.html

[10] Fermilab Document ESE-SVX-950719, October 2001.

[11] Honeywell, Kansas City Plant.

http://www.honeywell.com/sites/kcp/

[12] Finisar.

http://finisar.com//

[13] Micron Semiconductor Ltd., 1 Royal Buildings, Marlborough Road, Lancing, Sussex, BN15 8UN, England.

URL http://www.micronsemiconductor.co.uk/.

[14] ELMA:Research and Production Organization for Electronic Materials (NPO Elma), 103460 Moscow, Russia.

[15] Canberra Eurisys, 4 Avenue des Frenses, 78067 St. Quentin Yvelines, CEDEX, France.

URL http://www.eurisysmesures.com/.

[16] T. Ohsugi, al., Micro-Discharges of AC-Coupled Silicon Strip Sensors (International Symposium on Development and Application of Semiconductor Tracking Detectors, Hiroshima, Japan, 1993).

[17] M. Moll, PhD Thesis, Radiation damage in silicon particle detectors: Microscopic defects and macroscopic properties, DESY-THESIS1999-040. 
[18] Brush-Wellman, Cleveland, OH, U.S.A

[19] Ablefilm $563 \mathrm{~K}$ Ablestik, USA

[20] The D0 SMT Production Testing Group, Electrical Production Testing of the D0 Silicon Microstrip Tracker Detector Modules, FERMILAB-TM-2348-E.

[21] LPC, Litchfield, MN, USA.

[22] Dyconex AG, Bassersdorf, Switzerland.

URL http://www.dyconex.com/.

[23] Speedy Circuits, Huntington Beach, CA, USA.

URL http://www.speedycircuits.com/

[24] Compunetics Inc., Monroeville, PA, USA.

URL http://www.compunetics.com/.

[25] User Manual, BiRa Systems, "Model VME 4877PS High Voltage Power Supply System Manual 2nd Edition March 1998

URL http://www.dsp-fpga.com/products/search/fm/id/?13899

[26] L. Bagby et. al, SVX/silicon detector studies. FERMILAB-CONF-95-351

[27] Phoenix Precision, Phoenix, AZ, USA.

[28] R. Wunstorf, Radiation Hardness of Silicon Detectors: Current Status, IEEE Transactions on Nuclear Science, Vol. 44, No. 3, June 1997. 